\title{
Singular mean field equations on compact Riemann surfaces
}

\author{
Pierpaolo Esposito* and Pablo Figueroa ${ }^{\dagger}$
}

October 21, 2018

\begin{abstract}
For a general class of elliptic PDE's in mean field form on compact Riemann surfaces with exponential nonlinearity, we address the question of the existence of solutions with concentrated nonlinear term, which, in view of the applications, are physically of definite interest. In the model, we also include the possible presence of singular sources in the form of Dirac masses, which makes the problem more difficult to attack.
\end{abstract}

Keywords:

2000 AMS Subject Classification:

\section{Introduction}

Let us consider the problem

$$
-\Delta_{g} u=\lambda\left(\frac{k e^{u}}{\int_{S} k e^{u} d v_{g}}-\frac{1}{|S|}\right)
$$

on a compact, orientable Riemann surface $(S, g)$, where $\lambda>0, k$ is a smooth function and $|S|$ is the area of $S$. Here, $\Delta_{g}$ is the Laplace-Beltrami operator and $d v_{g}$ is the area element in $(S, g)$.

Equation (11) and its variants arise in many different contexts. The Nirenberg problem concerns the existence on $\mathbb{S}^{2}$ of metrics - conformal to the standard round metric $g_{0}$ - with Gaussian curvature $k$, and corresponds to equation (11) with $\lambda=8 \pi$. Indeed, a solution $u$ of (1) on $\left(\mathbb{S}^{2}, g_{0}\right)$ with $\lambda=8 \pi$ provides a metric $\frac{4 \pi e^{u}}{\int_{\mathrm{s}^{2}} k e^{u} d v_{g_{0}}} g_{0}$, conformal to $g_{0}$, with Gaussian curvature $k$. For a general compact Riemann surface, the prescribed Gaussian curvature problem is referred to as the Kazdan-Warner problem. Since there are plenty of results in literature, let us just quote the ones due to Kazdan and Warner [35, Chang and Yang [13] and Chang, Gursky, Yang [12. For bounded domains of $\mathbb{R}^{2}$, a variant of (1) with Dirichlet boundary condition arises in fluid mechanics as the equation for the stream function of a turbulent Euler flow with vortices of same orientation. By a statistical mechanics approach a rigorous derivation of it can be given as the mean-field limit of the Onsager's vortex theory, as shown by Kiessling [14, 36] and Caglioti, Lions, Marchioro, Pulvirenti [9, 10, and it is referred to as the "mean field equation". In all these contexts, the function $k$ is typically positive.

\footnotetext{
*Dipartimento di Matematica, Università degli Studi "Roma Tre", Largo S. Leonardo Murialdo 1, 00146 Roma, Italy, e-mail: esposito@mat.uniroma3.it. Author supported by Prin project "Critical Point Theory and Perturbative Methods for Nonlinear Differential Equations" and FIRB-IDEAS project "Analysis and Beyond".

†Departamento de Matemática, Pontificia Universidad Católica de Chile, Avenida Vicuña Mackenna 4860, Macul, Santiago, Chile. E-mail: pfigueros@mat.puc.cl. Author supported by grants Becas de Pasantías Doctorales en el Extranjero BECAS-CHILE and Fondecyt Postdoctorado 3120039, Chile.
} 
Notice that (11) contains also the singular mean-field equation

$$
-\Delta_{g} v=\lambda\left(\frac{h e^{v}}{\int_{S} h e^{v} d v_{g}}-\frac{1}{|S|}\right)+\frac{4 \pi N}{|S|}-4 \pi \sum_{j=1}^{l} n_{j} \delta_{p_{j}} \quad \text { in } S
$$

as a special case, where $h>0, p_{j} \in S, j=1, \ldots, l$, are distinct points, $n_{j}>0$ and $N=\sum_{j=1}^{l} n_{j}$. Indeed, introducing the Green function $G(x, p)$ with pole at $p \in S$ as the solution of

$$
\left\{\begin{array}{l}
-\Delta_{g} G(\cdot, p)=\delta_{p}-\frac{1}{|S|} \quad \text { in } S \\
\int_{S} G(x, p) d v_{g}=0
\end{array}\right.
$$

the function $u(x)=v(x)+4 \pi \sum_{j=1}^{l} n_{j} G\left(x, p_{j}\right)$ does solve (1) with $k(x)=h(x) e^{-4 \pi \sum_{j=1}^{l} n_{j} G\left(x, p_{j}\right)}$.

Here, the function $k$ is no longer positive, but is still nonnegative with zero set $\left\{p_{1}, \ldots, p_{l}\right\}$. On a flat torus $T$, singular mean-field equations with integer multiplicities $\left\{n_{1}, \ldots, n_{l}\right\} \subset \mathbb{N}$ arise in the study of the asymptotics for non-topological (stationary) condensates in the relativistic abelian Chern-Simons-Higgs model as the Chern-Simons parameter tends to zero, as shown by Nolasco and Tarantello [46. In the context of Euler flows, the presence of singular sources model the interaction of the fluid running on the given surface $S$ with sinks of given vorticities and opposite orientation w.r.t. all the vortices present in the fluid.

Observe that (10) admits a variational structure, in the sense that weak solutions for (1) are critical points of the following energy functional

$$
J_{\lambda}(u)=\frac{1}{2} \int_{S}|\nabla u|_{g}^{2} d v_{g}-\lambda \log \left(\int_{S} k e^{u} d v_{g}\right), u \in \bar{H},
$$

where $\bar{H}=\left\{u \in H^{1}(S): \int_{S} u d v_{g}=0\right\}$. For $\lambda<8 \pi, J_{\lambda}$ is bounded from below and the infimum of $J_{\lambda}$ is achieved by the well-known Moser-Trudinger inequality.

Let us focus first on the regular case $k>0$. For $k=1$ Struwe and Tarantello [47] were able to obtain non-trivial solutions of (11) for $8 \pi<\lambda<4 \pi^{2}$ on the square flat torus $T$. In the case of compact Riemann surfaces with genus $g \geq 1$ the existence of solutions for (11) with $8 \pi<\lambda<16 \pi$ was shown by Ding, Jost, Li, Wang [29] still by a variational approach. The case $S=\mathbb{S}^{2}$ of zero genus was considered by Lin [39] who proved nonvanishing of the Leray-Schauder degree $d_{\lambda}$ associated to (1) for $8 \pi<\lambda<16 \pi$ (and $d_{\lambda}=0$ for $16 \pi<\lambda<24 \pi$ ).

Since the solutions set of (1) is bounded in $C^{2, \alpha}(S), \alpha \in(0,1)$, as long as $\lambda$ is far from the critical parameter's range $8 \pi \mathbb{N}$, the degree $d_{\lambda}$ is well-defined and constant for all $\lambda \in(8 \pi m, 8 \pi(m+1))$, $m \in \mathbb{N}$. As observed by Y.Y. Li 38, its value should just depend on $m$ and the topology of $S$. The program for computing $d_{\lambda}$, initiated in [38, was completely settled by Chen and Lin [16] showing that

$$
d_{\lambda}=\left(\begin{array}{c}
m-\chi(S) \\
m
\end{array}\right)
$$

where $\chi(S)=2-2 g$ is the Euler characteristic of $S$ (see also the variational approach later developed by Malchiodi [43]). For $S \neq \mathbb{S}^{2}$, the degree $d_{\lambda}$ is always non-trivial yielding to a solution of (11) for all $\lambda \notin 8 \pi \mathbb{N}$. While, as already partially proved by Lin [39], for $\mathbb{S}^{2}$ there holds $d_{\lambda}=0$ for all $\lambda>16 \pi$ with $\lambda \notin 8 \pi \mathbb{N}$, and no existence statements can be deduced. A complete positive answer to the existence issue for (1) has been provided by Djadli 30 for all $\lambda \notin 8 \pi \mathbb{N}$ by means of a variational approach of min-max type, inspired by the result of Djadli and Malchiodi 31 concerning the fourth-order Paneitz operator in conformal geometry. Multiplicity results have been provided by De Marchis 26, 27. 
Solutions of (11) are no longer a pre-compact set when $\lambda \rightarrow 8 \pi \mathbb{N}$ : blow-up in $L^{\infty}$-norm along with the concentration of the measure $\lambda \frac{k e^{u}}{\int_{S} k e^{u} d v_{g}}$ as a sum of Dirac masses possibly arise for sequences of solutions as $\lambda \rightarrow 8 \pi \mathbb{N}$. Since $d_{\lambda}$ can change just when $\lambda$ crosses the values $8 \pi m$, $m \in \mathbb{N}$, it is crucial to have a precise asymptotic knowledge of blow-up solutions $u_{\lambda}$ and uniquely characterize them as $\lambda \rightarrow 8 \pi m$. The most refined asymptotic analysis is given by Chen and Lin [15: in particular, as $\lambda \rightarrow 8 \pi m u_{\lambda}$ has $m$ well-separated maximum points (up to a subsequence) which converge to a critical point in $S^{m} \backslash \Delta$ of

$$
\varphi_{m}(\xi)=\frac{1}{4 \pi} \sum_{j=1}^{m} \log k\left(\xi_{j}\right)+\sum_{j=1}^{m} H\left(\xi_{j}, \xi_{j}\right)+\sum_{l \neq j} G\left(\xi_{l}, \xi_{j}\right),
$$

where $H(x, \xi)$ is the regular part of $G(x, \xi)$ and $\Delta=\left\{\xi \in S^{m}: \xi_{i}=\xi_{j}\right.$ for $\left.i \neq j\right\}$ is the diagonal set in $S^{m}$. Let us notice that a critical point $\xi$ of $\varphi_{m}$ does satisfy

$$
\left.\nabla\left[\log k(x)+8 \pi H\left(x, \xi_{i}\right)+8 \pi \sum_{j \neq i} G\left(x, \xi_{j}\right)\right]\right|_{x=\xi_{i}}=0
$$

for all $i=1, \ldots, m$. In [16 blow-up solutions are constructed and their contribution to the degree is computed, so to determine (by local uniqueness of blow-up solutions) the jump in the values of $d_{\lambda}$ across $\lambda=8 \pi \mathrm{m}$. Since the degree $d_{\lambda}$ does not depend on $k$, it is possible to choose a positive function $k$ so that all the c.p.'s of $\varphi_{m}$ are non-degenerate, and then in [16] the authors simply address the existence of blow-up sequences of solutions for (11) which concentrate at non-degenerate c.p.'s of $\varphi_{m}$ as $\lambda \rightarrow 8 \pi m$.

The aim of the present paper is twofold. On one hand, we are interested in the construction of blow-up solutions with a general potential $k$ for which the corresponding $\varphi_{m}$ can possibly have degenerate but "stable" c.p.'s. On the other hand, we are interested to the singular mean-field equation or, equivalently, to (1) with a nonnegative potential $k$ which vanishes somewhere.

Let us focus now on the singular case. The first asymptotic analysis has been carried out by Bartolucci and Tarantello [7, with an application in the electroweak theory following [29]. The asymptotic analysis has been refined later in [2, 17, with the on-going project by Chen and Lin [18] of computing the Leray-Schauder degree $d_{\lambda}, \lambda \notin \Lambda$, where

$$
\Lambda=8 \pi \mathbb{N}+\left\{8 \pi \sum_{j \in J}\left(1+n_{j}\right): J \subset\{1, \ldots, l\}\right\}
$$

is the correponding critical set of parameters where compactness might fail, see [7]. For $n_{j} \geq 1$ the degree $d_{\lambda}$ has been computed by Chen, Lin and Wang [19] for $\lambda \in(8 \pi, 16 \pi)$, revealing the special role played by $\mathbb{S}^{2}$, the sphere being the only surface for which the degree can vanish (precisely, it vanishes only for $l=1$ ). The critical regime $\lambda=8 \pi$ has been considered in [28, 45. for a general surface. However, as we will explain below, the problem on the torus with total multiplicity $N=2$ becomes more degenerate. In this case, existence/non-existence issues have been discussed in [19] for a rectangular torus (along with the computation of $d_{8 \pi}$ ) and in [40] for the general case, physically relevant issues in connection with non-topological 2 -condensates in the Chern-Simons-Higgs model [46. Existence results have been recently obtained by means of a variational approach of min-max type, inspired by [29, 31], confirming the special role of $\mathbb{S}^{2}$ (see also the discussion in [5, 50]). For $\lambda \notin \Lambda$, the singular problem (2) is solvable for $S \neq \mathbb{S}^{2}$ [4] (see also [3] for an application in the electroweak theory). The case of the sphere has been first considered by Malchiodi and Ruiz [44] for $n_{j} \leq 1$ and $\lambda \in(8 \pi, 16 \pi) \backslash \Lambda$ : the crucial assumption to have existence for (2) is that $\# J \neq 1$, where $J=\left\{j=1, \ldots, l: \lambda<8 \pi\left(1+n_{j}\right)\right\}$. The result has been extended by Bartolucci and Malchiodi [6] to general $n_{j}$ 's and $\lambda$ under the condition $l \geq 2$ and $\lambda<8 \pi \min \left\{1+n_{j}: j=1, \ldots, l\right\}$, corresponding to the situation $\# J=l$.

In some of the above-mentioned papers, the regular/singular mean field equation has been also considered on a bounded domain $\Omega \subset \mathbb{R}^{2}$ with homogeneous Dirichlet b.c. Since $\int_{S} k e^{u_{\lambda}} d v_{g} \rightarrow$ 
$+\infty$ along any non-compact sequence of solutions $u_{\lambda}$ for (10), through the setting $\rho=\frac{\lambda}{\int_{S} k e^{u} d v_{g}}$ problem (11) is naturally related (but not equivalent) to $-\Delta_{g} u=\rho\left(k e^{u}-\frac{1}{|S|} \int_{S} k e^{u} d v_{g}\right)$ with $\rho \rightarrow 0^{+}$, which has been recently studied by the second author in 34. Blow-up solutions for the corresponding Dirichlet problem

$$
\begin{cases}-\Delta u=\rho k e^{u} & \text { in } \Omega \\ u=0 & \text { on } \partial \Omega\end{cases}
$$

on a bounded domain $\Omega \subset \mathbb{R}^{2}$ have been constructed at c.p.'s of $\varphi_{m}$ which are non-degenerate [1] or, more generally, "stable" 25, 33. A "stable" critical value for $\varphi_{m}$ has been constructed by del Pino, Kowalczyk and Musso 25] for the regular problem on a non-simply connected domain and for the singular problem with $l=1$. The latter case has been extended to the flat torus [34, and a similar result is still in order for multiple singular sources as shown by D'Aprile 21, under suitable relations between $m$ and the $n_{j}$ 's.

Setting

$$
\rho_{j}(x)=k(x) e^{8 \pi H\left(x, \xi_{j}\right)+8 \pi \sum_{l \neq j} G\left(x, \xi_{l}\right)},
$$

for $\xi \in S^{m} \backslash \Delta$ we introduce the notation

$$
A(\xi)=4 \pi \sum_{j=1}^{m}\left[\Delta_{g} \rho_{j}\left(\xi_{j}\right)-2 K\left(\xi_{j}\right) \rho_{j}\left(\xi_{j}\right)\right],
$$

where $K$ is the Gaussian curvature of $(S, g)$. Letting $\tilde{S}=\{k>0\}$, our first main result is

Theorem 1.1. Let $\mathcal{D} \subset \subset \tilde{S}^{m} \backslash \Delta$ be a stable critical set of $\varphi_{m}$. Assume that $A(\xi)>0(<0$ resp.) for all $\xi \in \mathcal{D}$. Then, for all $\lambda$ in a small right (left resp.) neighborhood of $8 \pi m$ there is a solution $u_{\lambda}$ of (11) so that (along sub-sequences)

$$
\frac{\lambda k e^{u_{\lambda}}}{\int_{S} k e^{u_{\lambda}} d v_{g}} \rightarrow 8 \pi \sum_{j=1}^{m} \delta_{q_{j}}
$$

as $\lambda \rightarrow 8 \pi m$ in the sense of measures in $S$, for some $q=\left(q_{1}, \ldots, q_{m}\right) \in \mathcal{D}$.

Along with (8) notice that there always hold that $u_{\lambda}-\log \int_{S} k e^{u_{\lambda}} d v_{g} \rightarrow-\infty$ in $C_{\operatorname{loc}}(S \backslash$ $\left.\left\{q_{1}, \ldots, q_{m}\right\}\right)$ and

$$
\sup _{U_{j}}\left(u_{\lambda}-\log \int_{S} k e^{u_{\lambda}} d v_{g}\right) \rightarrow+\infty
$$

as $\lambda \rightarrow 8 \pi m$, for any neighborhood $U_{j}$ of $q_{j}$ in $S, j=1, \ldots, m$. The notion of stability we are using here is the one introduced in [37]:

Definition 1.2. A critical set $\mathcal{D} \subset \subset \tilde{S}^{m} \backslash \Delta$ of $\varphi_{m}$ is stable if for any closed neighborhood $U$ of $\mathcal{D}$ in $\tilde{S}^{m} \backslash \Delta$ there exists $\delta>0$ such that, if $\left\|G-\varphi_{m}\right\|_{C^{1}(U)} \leq \delta$, then $G$ has at least one critical point in $U$. In particular, the minimal/maximal set of $\varphi_{m}$ is stable (if $\varphi_{m}$ is not constant) as well as any isolated c.p. of $\varphi_{m}$ with non-trivial local degree.

Since $A(\xi)$ can be re-written as

$$
\begin{aligned}
A(\xi) & =4 \pi \sum_{j=1}^{m} \rho_{j}\left(\xi_{j}\right)\left[\Delta_{g} \log \rho_{j}\left(\xi_{j}\right)+\left|\nabla \log \rho_{j}\left(\xi_{j}\right)\right|_{g}^{2}-2 K\left(\xi_{j}\right)\right] \\
& =4 \pi \sum_{j=1}^{m} \rho_{j}\left(\xi_{j}\right)\left[\Delta_{g} \log k\left(\xi_{j}\right)+\frac{8 \pi m}{|S|}+\left|\nabla \log \rho_{j}\left(\xi_{j}\right)\right|_{g}^{2}-2 K\left(\xi_{j}\right)\right],
\end{aligned}
$$


for a c.p. of $\varphi_{m}$ we have that

$$
A(\xi)=4 \pi \sum_{j=1}^{m} \rho_{j}\left(\xi_{j}\right)\left[\Delta_{g} \log k\left(\xi_{j}\right)+\frac{8 \pi m}{|S|}-2 K\left(\xi_{j}\right)\right]
$$

in view of $\nabla \rho_{j}\left(\xi_{j}\right)=0$ for all $j=1, \ldots, m$. Since for $k>0$ the function $\varphi_{m}$ always attains its minimum value in $S^{m} \backslash \Delta$ and the minimal set is clearly stable, as a first by-product we have (see also [16]):

Corollary 1.3. Assume $k>0$. Let $m \in \mathbb{N}$ be so that either $1 \leq m<\inf _{S} \frac{|S|}{8 \pi}\left[2 K-\Delta_{g} \log k\right]$ or $m>\sup _{S} \frac{|S|}{8 \pi}\left[2 K-\Delta_{g} \log k\right]$. Then there exist solutions $u_{\lambda}$ of (1) which concentrate at $m$ points $q_{1}, \ldots, q_{m}$ in the sense (8) as $\lambda \rightarrow 8 \pi m$, where $q=\left(q_{1}, \ldots, q_{m}\right)$ is a minimum point of $\varphi_{m}$ in $S^{m} \backslash \Delta$.

When the surface $(S, g)$ has constant Gaussian curvature, by the Gauss-Bonnet formula we have that $K=\frac{2 \pi \chi(S)}{|S|}$. For $k=1$, Corollary 1.3 then provides the existence of blow-up solutions $u_{\lambda}$ concentrating at $m$ points as $\lambda \rightarrow 8 \pi m$ for all $m \geq 2$, where $\lambda$ belongs to a small right neighborhood of $8 \pi m$. The case $m=1$ is problematic since $\varphi_{1}$ is a constant function.

Concerning the singular problem (2), in general the function $\varphi_{m}$ has neither maximum nor minimum points, and it is then natural to search for saddle critical points. The min-max scheme introduced in 21] works in the Euclidean context as well as in the case of a surface 22]. In particular, on $\mathbb{S}^{2}$ the function $\varphi_{m}$ has a "stable" critical value of min-max type as soon as $l \geq 2$ and

$$
8 \pi m \notin 8 \pi \mathbb{N}+8 \pi\left(1+n_{j}\right) \forall j=1, \ldots, l, \quad \# J \geq 2,
$$

where $J=\left\{j=1, \ldots, l: 8 \pi m<8 \pi\left(1+n_{j}\right)\right\}$. In the construction, each singular source $p_{i}$ has to be coupled with some $p_{j} \neq p_{i}$ in order to deform $\mathbb{S}^{2} \backslash\left\{p_{i}, p_{j}\right\}$ onto a circle running around $p_{i}$, and the condition $l \geq 2$ is crucial. Notice that the min-max scheme provides a critical point $q$ of $\varphi_{m}$ so that $\{q\}$ is a stable critical set according to Definition 1.2. Morover, since \#J $\geq 2$ yields to $2 m<2+N$, for $k(x)=e^{-4 \pi \sum_{j=1}^{l} n_{j} G\left(x, p_{j}\right)}$ we have that

$$
A(q)=\frac{16 \pi^{2}}{\left|\mathbb{S}^{2}\right|} \sum_{j=1}^{m} \rho_{j}\left(q_{j}\right)[-N+2 m-2]<0
$$

in view of $K=\frac{4 \pi}{\left|\mathbb{S}^{2}\right|}$. As a second by-product of Theorem 1.1, we have:

Corollary 1.4. Let $h=1$ and $l \geq 2$. Assume that $S$ is topologically a sphere and that $m$ satisfies (10). Then, for all $\lambda$ in a small left neighborhood of $8 \pi m$ there is a solution $u_{\lambda}$ of (2) which concentrates at $m$ points $q_{1}, \ldots, q_{m}$ in the sense (8) as $\lambda \rightarrow 8 \pi m$.

Theorem 1.1 and Corollary 1.3 are the perturbative counter-parts of global existence results already available in literature, obtained via degree theory or a variational approach. However, the behavior of such solutions as $\lambda \rightarrow 8 \pi m$ is not known whereas the ones we construct exhibit blow-up phenomena, a property that has a definite interest in its own. More important, Corollary 1.4 gives completely new results for the case of $\mathbb{S}^{2}$, by showing that in a perturbative regime the condition $\# J \geq 2$ in $[44$ is sufficient for the existence in the general case, beyond the results in [6]. Moreover, in 22] the cases $\# J=0,1$ are also treated.

There are cases for which $A(\xi)$ can vanish. By invariance under rotations, it is easily seen that on $\mathbb{S}^{2}$ the function $H(\xi, \xi)$ is constant, and then the c.p.'s of $\varphi_{1}$ and $k$ do coincide. Since in particular $\left.\nabla H(x, \xi)\right|_{x=\xi}=0$, by (9) for $S=\mathbb{S}^{2}$ and $m=1$ the coefficient $A(\xi)$ writes as

$$
A(\xi)=4 \pi k(\xi) e^{8 \pi H(\xi, \xi)}\left[\Delta_{g} \log k(\xi)+|\nabla \log k(\xi)|_{g}^{2}\right]=4 \pi e^{8 \pi H(\xi, \xi)} \Delta_{g} k(\xi)
$$


and might vanish at some c.p. of $k$. Another typical example is the singular mean-field equation (2) on the flat torus $T$ with $h=1$ and even total multiplicity $N$ : since $k=e^{-4 \pi \sum_{j=1}^{l} n_{j} G\left(x, p_{j}\right)}$ and $K \equiv 0$, by (9) the coefficient $A(\xi)$ writes for $m=\frac{N}{2}$ as

$$
A(\xi)=4 \pi \sum_{j=1}^{m} \rho_{j}\left(\xi_{j}\right)\left|\nabla \log \rho_{j}\left(\xi_{j}\right)\right|_{g}^{2}=(4 \pi)^{3} \sum_{j=1}^{m} \rho_{j}\left(\xi_{j}\right)\left|\nabla_{\xi_{j}} \varphi_{m}(\xi)\right|_{g}^{2} \geq 0
$$

and vanishes exactly at the c.p.'s of $\varphi_{m}$. In all these situations, a more refined analysis is necessary.

Introduce the following quantity

$$
\begin{aligned}
B(\xi)= & -2 \pi \sum_{j=1}^{m}\left[\Delta_{g} \rho_{j}\left(\xi_{j}\right)-2 K\left(\xi_{j}\right) \rho_{j}\left(\xi_{j}\right)\right] \log \rho_{j}\left(\xi_{j}\right)-\frac{A(\xi)}{2} \\
& +\lim _{r \rightarrow 0}\left[8 \int_{S \backslash \cup_{j=1}^{m} B_{r}\left(\xi_{j}\right)} k e^{8 \pi \sum_{j=1}^{m} G\left(x, \xi_{j}\right)} d v_{g}-\frac{8 \pi}{r^{2}} \sum_{j=1}^{m} \rho_{j}\left(\xi_{j}\right)-A(\xi) \log \frac{1}{r}\right],
\end{aligned}
$$

where $B_{r}(\xi)$ denotes the pre-image of $B_{r}(0)$ through the isothermal coordinate system at $\xi$. The quantity $B(\xi)$ has been first used and derived by Chang, Chen and Lin [11] in the study of the mean field equation on bounded domains (see also [19, 42] for the case of the torus). We have the following general result, of which Theorem 1.1 is just a special case:

Theorem 1.5. Let $\mathcal{D} \subset \subset \tilde{S}^{m} \backslash \Delta$ be a stable critical set of $\varphi_{m}$. Assume that

$$
\text { either } A(\xi)>0(<0 \text { resp. }) \quad \text { or } \quad A(\xi)=0, B(\xi)>0(<0 \text { resp. })
$$

do hold in a closed neighborhood $U$ of $\mathcal{D}$ in $\tilde{S}^{m} \backslash \Delta$. Then, for all $\lambda$ in a small right (left resp.) neighborhood of $8 \pi m$ there is a solution $u_{\lambda}$ of (1) which concentrates (along sub-sequences) at $m$ points $q_{1}, \ldots, q_{m}$ in the sense (8) as $\lambda \rightarrow 8 \pi m$, for some $q \in \mathcal{D}$.

To deal a with stable critical set $\mathcal{D}$ in the sense above, we need to require condition (13) on a neighborhood of $\mathcal{D}$. In case we strengthen the stability assumption, we can relax the assumption (13) to hold just on $\mathcal{D}$. As an instructive example, in Remark 4.5)(i) we present the case of a non-degenerate local minimum/maximum point.

We can now discuss the two previous examples for which the coefficient $A(\xi)$ vanishes. For $S=\mathbb{S}^{2}$ and $m=1$, there holds $\varphi_{1}=\frac{1}{4 \pi} \log k+$ const. since $H(\xi, \xi)=$ const.. In view of (11), assume that $\Delta_{g} k \geq 0$ in a small neighborhood $U$ of the minimal set $\mathcal{D}=\left\{\xi \in S: \varphi_{1}(\xi)=\min _{S} \varphi_{1}\right\}$ so to have $A(\xi) \geq 0$ in $U$. We just need to show that $B(\xi)>0$ in $U$ so to use Theorem 1.5 with $\mathcal{D}$, which is clearly a stable critical set of $\varphi_{1}$ as soon as $k$ is not a constant function. Up to take $U$ smaller, it is clearly enough to show that $B(\xi)>0$ for all $\xi \in \mathcal{D}$ with $A(\xi)=0$. Up to a rotation, we can assume that $\xi$ is the south pole $P$ of $\mathbb{S}^{2}$. The stereographic projection $\pi:(x, y, z) \rightarrow\left(\frac{2 x}{1-z}, \frac{2 y}{1-z}\right)$ through the north pole is an isometry between ( $\mathbb{S}^{2} \backslash\{$ north pole $\left.\}, g_{0}\right)$ and $\left(\mathbb{R}^{2}, \frac{16}{\left(4+u^{2}+v^{2}\right)^{2}} \delta_{\text {eucl }}\right)$. Since it is easily seen that $\operatorname{dist}\left(\pi^{-1}(u, v), P\right)=|(u, v)|$ and

$$
G\left(\pi^{-1}(u, v), P\right)=-\frac{1}{2 \pi} \log |(u, v)|+\frac{1}{4 \pi} \log \left(4+u^{2}+v^{2}\right)+c_{0},
$$

in the coordinate system $\pi$ in terms of $\tilde{k}(u, v)=k\left(\pi^{-1}(u, v)\right)$ we can write that

$$
B(P)=128 e^{c_{0}} \lim _{r \rightarrow 0} \int_{\mathbb{R}^{2} \backslash B_{r}(0)} \frac{\tilde{k}(u, v)-\tilde{k}(0,0)}{\left(u^{2}+v^{2}\right)^{2}} d u d v>0
$$

in view of $k \geq k(P), k \neq k(P)$. Similarly, we can treat the case in which $\Delta_{g} k \leq 0$ does hold in a small neighborhood $U$ of the maximal set $\mathcal{D}$. 
In the case of the flat torus $T$ with $N$ even, $m=\frac{N}{2}$ and $k=e^{u_{0}}, u_{0}=-4 \pi \sum_{j=1}^{l} n_{j} G\left(x, p_{j}\right)$, at a c.p. $\xi$ of $\varphi_{m}$ the coefficient

$$
B(\xi)=\lim _{r \rightarrow 0}\left[8 \int_{T \backslash \cup_{j=1}^{m} B_{r}\left(\xi_{j}\right)} e^{u_{0}+8 \pi \sum_{j=1}^{m} G\left(x, \xi_{j}\right)} d x-\frac{8 \pi}{r^{2}} \sum_{j=1}^{m} \rho_{j}\left(\xi_{j}\right)\right]
$$

can be re-written in the following way:

- if $N=2, m=1$

$$
B(\xi)=8 e^{u_{0}(\xi)+8 \pi H(\xi, \xi)}\left[\int_{T} \frac{e^{u_{0}(x)-u_{0}(\xi)+8 \pi H(x, \xi)-8 \pi H(\xi, \xi)}-1}{|x-\xi|^{4}} d x-\int_{\mathbb{R}^{2} \backslash T} \frac{d x}{|x-\xi|^{4}}\right],
$$

where the integral on $T$ is conditionally convergent in view of $\left.\nabla\left(u_{0}(x)+8 \pi H(x, \xi)\right)\right|_{x=\xi}=0$ and $\Delta\left(u_{0}(x)+8 \pi H(x, \xi)\right) \equiv 0$;

- if $N \geq 4$ even, $m=\frac{N}{2}$

$$
B(\xi)=8 \sum_{j=1}^{m}\left[\int_{T_{j}} \frac{\rho_{j}(x)-\rho_{j}\left(\xi_{j}\right)}{\left|x-\xi_{j}\right|^{4}} d x-\rho_{j}\left(\xi_{j}\right) \int_{\mathbb{R}^{2} \backslash T_{j}} \frac{d x}{\left|x-\xi_{j}\right|^{4}}\right],
$$

where $T$ has been splitted into disjoint sets $T_{1}, \ldots, T_{m}$ so that $B_{r}\left(\xi_{j}\right) \subset T_{j}$ for $r$ small and all $j$.

When $T$ is a rectangle, $l=1$ and $n_{1}=2$, the constant $B(\xi)$ has been used by Chen, Lin and Wang [19] in the computation of the degree $d_{8 \pi}$. The function $\varphi_{1}=\frac{u_{0}}{4 \pi}+$ const. has exactly three nondegenerate critical points $\xi_{1}, \xi_{2}$ (saddle points) and $\xi_{3}$ (maximum point) with $B\left(\xi_{1}\right), B\left(\xi_{2}\right)>0$ and $B\left(\xi_{3}\right)<0$. By Theorem 1.5 and Remark 4.5. (i) we deduce the existence of

- two distinct families of solutions, for $\lambda$ in a small right neighborhood of $8 \pi$, concentrating at $\xi_{1}$ and $\xi_{2}$ as $\lambda \rightarrow 8 \pi$;

- one family of solutions, for $\lambda$ in a small left neighborhood of $8 \pi$, concentrating at $\xi_{3}$ as $\lambda \rightarrow 8 \pi$.

Moreover, $B(\xi)$ has been recently used in the construction of non-topological condensates for the relativistic abelian Chern-Simons-Higgs model as the Chern-Simons parameter tends to zero, see [42]. Unfortunately, when $N \geq 4$ there are no examples where the sign of $B(\xi)$ can be determined.

To explain more clearly such a connection, recall that in the relativistic abelian Chern-SimonHiggs model the $N$ vortex-condensates are gauge-periodic stationary matter configurations with finite-energy that, in the self-dual regime, express in terms of solutions for

$$
-\Delta w=\frac{1}{\epsilon^{2}} e^{w}\left(1-e^{w}\right)-4 \pi \sum_{j=1}^{l} n_{j} \delta_{p_{j}}
$$

in a flat torus $T$. We refer to 32 for a complete account on the model and to [49] for the analytical results concerning it. The quantity $2 \epsilon>0$ is the Chern-Simons parameter, $p_{j} \in S, j=1, \ldots, l$, are distinct points and $n_{j} \in \mathbb{N}$. Physically, $\epsilon$ is very small and two classes of solutions are relevant: either $e^{w} \rightarrow 1$ as $\epsilon \rightarrow 0^{+}$("topological" type) or $e^{w} \rightarrow 0$ as $\epsilon \rightarrow 0^{+}$("non-topological" type). Topological solutions were first found by Caffarelli and Yang [ $]$. However, non-topological condensates represent the main feature of the Chern-Simons-Higgs model which were absent in the classical (Maxwell-Higgs) vortex theory, whose existence was established by Tarantello [48. 
Through the change $w \rightarrow w-u_{0}, u_{0}=-4 \pi \sum_{j=1}^{l} n_{j} G\left(x, p_{j}\right)$, the self-dual equation (14) reads equivalently as

$$
-\Delta w=\frac{1}{\epsilon^{2}} k e^{w}\left(1-k e^{w}\right)-\frac{4 \pi N}{|T|}
$$

with $k=e^{u_{0}}$. Setting $c=\frac{1}{|T|} \int_{T} w d x$ and $u=w-c \in \bar{H}$, an integration of (15) provides a relation between $c$ and $u$ (see [48]):

$$
e^{c} \int_{T} k e^{u} d x-e^{2 c} \int_{T} k^{2} e^{2 u} d x=4 \pi N \epsilon^{2} .
$$

Hence, necessarily

$$
u \in \mathcal{A}_{\epsilon}=\left\{u \in \bar{H} \mid\left(\int_{T} k e^{u}\right)^{2}-16 \pi N \epsilon^{2} \int_{T} k^{2} e^{2 u} \geq 0\right\}
$$

and then $c=c_{ \pm}(u)$ with

$$
e^{c_{ \pm}(u)}=\frac{8 \pi N \epsilon^{2}}{\int_{T} k e^{u} \mp \sqrt{\left(\int_{T} k e^{u}\right)^{2}-16 \pi N \epsilon^{2} \int_{T} k^{2} e^{2 u}}} .
$$

For solutions of "non-topological" type it is natural to choose $c_{-}(u)$, and then equation (15) reads in terms of $u \in \mathcal{A}_{\epsilon}$ as

$$
\begin{aligned}
-\Delta u= & 4 \pi N\left(\frac{k e^{u}}{\int_{T} k e^{u}}-\frac{1}{|T|}\right) \\
& +\frac{64 \pi^{2} N^{2} \epsilon^{2} \int_{T} k^{2} e^{2 u}}{\left(\int_{T} k e^{u}+\sqrt{\left(\int_{T} k e^{u}\right)^{2}-16 \pi N \epsilon^{2} \int_{T} k^{2} e^{2 u}}\right)^{2}}\left(\frac{k e^{u}}{\int_{T} k e^{u}}-\frac{k^{2} e^{2 u}}{\int_{T} k^{2} e^{2 u}}\right) .
\end{aligned}
$$

When $N$ is even and $m=\frac{N}{2}$, equation (16) is a perturbation of (11) $\lambda_{\lambda=8 \pi m}$ as $\epsilon \rightarrow 0^{+}$. The parallel becomes clear if we re-consider (11) itself as a perturbation of (1) $\lambda=8 \pi m$ as $\lambda \rightarrow 8 \pi m$. As far as (11) is concerned, the sign of the perturbation can be chosen since it depends on $\lambda-8 \pi m$. For (16) the sign of the perturbation is given and is like the case $\lambda<8 \pi m$ in which we need to require (13) with the negative sign $<0$. Even if we always have the wrong sign $A(\xi) \geq 0$, the coefficient $A(\xi)$ behaves like $\left|\nabla \varphi_{m}(\xi)\right|_{g}^{2}:=\sum_{j=1}^{m}\left|\nabla_{\xi_{j}} \varphi_{m}(\xi)\right|_{g}^{2}$ and, near a critical set $\mathcal{D}$ of $\varphi_{m}$, is very small. The condition $B(\xi)<0$ on $\mathcal{D}$ will then be enough, as stated in the following:

Theorem 1.6. Assume $N$ even. Let $\mathcal{D} \subset \subset\left(T \backslash\left\{p_{1}, \ldots, p_{l}\right\}\right)^{m} \backslash \Delta$ be a stable critical set of

$$
\varphi_{m}(\xi)=\frac{1}{4 \pi} \sum_{j=1}^{m} u_{0}\left(\xi_{j}\right)+\sum_{l \neq j} G\left(\xi_{l}, \xi_{j}\right) .
$$

Assume that $B(\xi)<0$ does hold in $\mathcal{D}$. Then, for all $\epsilon$ small there is a solution $w_{\epsilon}$ of (14) which concentrates at $m$ points $q_{1}, \ldots, q_{m}$, with $q=\left(q_{1}, \ldots, q_{m}\right) \in \mathcal{D}$, as $\epsilon \rightarrow 0$ in the sense of measures:

$$
\frac{1}{\epsilon^{2}} e^{w_{\epsilon}}\left(1-e^{w_{\epsilon}}\right)-8 \pi \sum_{j=1}^{m} \delta_{q_{j}}
$$

Correspondingly, there exist non-topological $N$ vortex-condensates of gauge potential $A_{\epsilon}$ and Higgs field $\phi_{\epsilon}$ for which the magnetic field $\left(F_{12}\right)_{\epsilon}$ is very concentrated at the $m$ points $q_{1}, \ldots, q_{m}$ (external to the so-called vortex-set $\left.\left\{p_{1}, \ldots, p_{l}\right\}\right)$ as $\epsilon \rightarrow 0$. 
Theorem 1.6 slightly improves the result in [42] (see [23, 24, 41] for concentration at the vortices) where they just deal with isolated c.p.'s of $\varphi_{m}$ with non-trivial local degree. Our "stability" assumption is more general as already explained in Definition 1.2. Even if $\varphi_{1}$ has always the maximal set as a "stable" critical set, a general existence result for 1 -point concentration does not follow since we don't know whether the coefficient $B(\xi)<0$ or not (apart from the case $l=1, n_{1}=2, T$ a rectangle).

\section{Approximation of the solution}

To construct approximating solutions of (1), the main idea is to use as "basic cells" the functions

$$
u_{\delta, \xi}(x)=u_{0}\left(\frac{|x-\xi|}{\delta}\right)-2 \log \delta \quad \delta>0, \xi \in \mathbb{R}^{2},
$$

where

$$
u_{0}(r)=\log \frac{8}{\left(1+r^{2}\right)^{2}} .
$$

They are all the solutions of

$$
\left\{\begin{array}{l}
\Delta u+e^{u}=0 \text { in } \mathbb{R}^{2} \\
\int_{\mathbb{R}^{2}} e^{u}<\infty
\end{array}\right.
$$

and do satisfy the following concentration property:

$$
e^{u_{\delta, \xi}} \rightarrow 8 \pi \delta_{\xi} \quad \text { in measure sense }
$$

as $\delta \rightarrow 0$. We will use now isothermal coordinates to pull-back $u_{\delta, \xi}$ in $S$.

Let us recall that every Riemann surface $(S, g)$ is locally conformally flat, and the local coordinates in which $g$ is conformal to the Euclidean metric are referred to as isothermal coordinates (see for example the simple existence proof provided by Chern [20]). For every $\xi \in S$ it amounts to find a local chart $y_{\xi}$, with $y_{\xi}(\xi)=0$, from a neighborhood of $\xi$ onto $B_{2 r_{0}}(0)$ (the choice of $r_{0}$ is independent of $\xi)$ in which $g=e^{\hat{\varphi}_{\xi}\left(y_{\xi}(x)\right)} d x$, where $\hat{\varphi}_{\xi} \in C^{\infty}\left(B_{2 r_{0}}(0), \mathbb{R}\right)$. In particular, $\hat{\varphi}_{\xi}$ relates with the Gaussian curvature $K$ of $(S, g)$ through the relation:

$$
\Delta \hat{\varphi}_{\xi}(y)=-2 K\left(y_{\xi}^{-1}(y)\right) e^{\hat{\varphi}_{\xi}(y)} \quad \text { for } y \in B_{2 r_{0}}(0) .
$$

We can also assume that $y_{\xi}, \hat{\varphi}_{\xi}$ depends smoothly in $\xi$ and that $\hat{\varphi}_{\xi}(0)=0, \nabla \hat{\varphi}_{\xi}(0)=0$.

We now pull-back $u_{\delta, 0}$ in $\xi \in S$, for $\delta>0$, by simply setting

$$
U_{\delta, \xi}(x)=u_{\delta, 0}\left(y_{\xi}(x)\right)=\log \frac{8 \delta^{2}}{\left(\delta^{2}+\left|y_{\xi}(x)\right|^{2}\right)^{2}}
$$

for $x \in y_{\xi}^{-1}\left(B_{2 r_{0}}(0)\right)$. Letting $\chi \in C_{0}^{\infty}\left(B_{2 r_{0}}(0)\right)$ be a radial cut-off function so that $0 \leq \chi \leq 1$, $\chi \equiv 1$ in $B_{r_{0}}(0)$, we introduce the function $P U_{\delta, \xi}$ as the unique solution of

$$
\left\{\begin{array}{l}
-\Delta_{g} P U_{\delta, \xi}(x)=\chi_{\xi}(x) e^{-\varphi_{\xi}(x)} e^{U_{\delta, \xi}(x)}-\frac{1}{|S|} \int_{S} \chi_{\xi} e^{-\varphi_{\xi}} e^{U_{\delta, \xi}} d v_{g} \quad \text { in } S \\
\int_{S} P U_{\delta, \xi} d v_{g}=0
\end{array}\right.
$$

where $\chi_{\xi}(x)=\chi\left(\left|y_{\xi}(x)\right|\right)$ and $\varphi_{\xi}(x)=\hat{\varphi}_{\xi}\left(y_{\xi}(x)\right)$. Notice that the R.H.S. in (18) has zero average and smoothly depends in $x$, and then (18) is uniquely solvable by a smooth solution $P U_{\delta, \xi}$.

Let us recall the transformation law for $\Delta_{g}$ under conformal changes: if $\tilde{g}=e^{\varphi} g$, then

$$
\Delta_{\tilde{g}}=e^{-\varphi} \Delta_{g} .
$$


Decompose now the Green function $G(x, \xi), \xi \in S$, as

$$
G(x, \xi)=-\frac{1}{2 \pi} \chi_{\xi}(x) \log \left|y_{\xi}(x)\right|+H(x, \xi),
$$

and by (3) then deduce that

$$
\left\{\begin{array}{l}
-\Delta_{g} H=-\frac{1}{2 \pi} \Delta_{g} \chi_{\xi} \log \left|y_{\xi}(x)\right|-\frac{1}{\pi}\left\langle\nabla \chi_{\xi}, \nabla \log \left|y_{\xi}(x)\right|\right\rangle_{g}-\frac{1}{|S|} \quad \text { in } S \\
\int_{S} H(\cdot, \xi) d v_{g}=\frac{1}{2 \pi} \int_{S} \chi_{\xi} \log \left|y_{\xi}(\cdot)\right| d v_{g} .
\end{array}\right.
$$

We have used that

$$
\Delta_{g} \log \left|y_{\xi}(x)\right|=\left.e^{-\hat{\varphi}_{\xi}(y)} \Delta \log |y|\right|_{y=y_{\xi}(x)}=2 \pi \delta_{\xi}
$$

in view of (19).

For $r \leq 2 r_{0}$ define $B_{r}(\xi)=y_{\xi}^{-1}\left(B_{r}(0)\right), A_{r}(\xi)=B_{r}(\xi) \backslash B_{r / 2}(\xi)$, and set

$$
f_{\xi}=\frac{\Delta_{g} \chi_{\xi}}{\left|y_{\xi}(x)\right|^{2}}+2\left\langle\nabla \chi_{\xi}, \nabla\left|y_{\xi}(x)\right|^{-2}\right\rangle_{g}+\frac{2}{|S|} \int_{\mathbb{R}^{2}} \frac{\chi^{\prime}(|y|)}{|y|^{3}} d y .
$$

By (22) it follows that

$$
\int_{S} f_{\xi} d v_{g}=\frac{1}{2 \delta^{2}} \int_{S} \Delta_{g} \Psi_{\delta, \xi} d v_{g}+O\left(\delta^{2}\right)=O\left(\delta^{2}\right)
$$

as $\delta \rightarrow 0$, where $\Psi_{\delta, \xi} \in H^{1}(S)$ is defined in (21). Thus, $\int_{S} f_{\xi} d v_{g}=0$, and then $F_{\xi}$ is well defined as the unique solution of

$$
\left\{\begin{array}{l}
-\Delta_{g} F_{\xi}=f_{\xi} \quad \text { in } S \\
\int_{S} F_{\xi} d v_{g}=0 .
\end{array}\right.
$$

We have the following asymptotic expansion of $P U_{\delta, \xi}$ as $\delta \rightarrow 0$ :

Lemma 2.1. The function $P U_{\delta, \xi}$ satisfies

$$
P U_{\delta, \xi}=\chi_{\xi}\left[U_{\delta, \xi}-\log \left(8 \delta^{2}\right)\right]+8 \pi H(x, \xi)+\alpha_{\delta, \xi}-2 \delta^{2} F_{\xi}+O\left(\delta^{4}|\log \delta|\right)
$$

uniformly in $S$, where $F_{\xi}$ is given in (20) and

$$
\alpha_{\delta, \xi}=-\frac{4 \pi}{|S|} \delta^{2} \log \delta+2 \frac{\delta^{2}}{|S|}\left(\int_{\mathbb{R}^{2}} \chi(|y|) \frac{e^{\hat{\varphi}_{\xi}(y)}-1}{|y|^{2}} d y+\pi-\int_{\mathbb{R}^{2}} \frac{\chi^{\prime}(|y|) \log |y|}{|y|} d y\right) .
$$

In particular, there holds

$$
P U_{\delta, \xi}=8 \pi G(x, \xi)-2 \frac{\delta^{2} \chi_{\xi}}{\left|y_{\xi}(x)\right|^{2}}+\alpha_{\delta, \xi}-2 \delta^{2} F_{\xi}+O\left(\delta^{4}|\log \delta|\right)
$$

locally uniformly in $S \backslash\{\xi\}$.

Proof: Let us define

$$
\Psi_{\delta, \xi}(x)=P U_{\delta, \xi}(x)-\chi_{\xi} \hat{U}_{\delta, \xi}-8 \pi H(x, \xi),
$$

where $\hat{U}_{\delta, \xi}=U_{\delta, \xi}-\log \left(8 \delta^{2}\right)$, for which there holds

$$
\int_{S} \Psi_{\delta, \xi} d v_{g}=-\int_{S}\left[\chi_{\xi} \hat{U}_{\delta, \xi}+8 \pi H(x, \xi)\right] d v_{g}=-\int_{S} \chi_{\xi} \log \frac{\left|y_{\xi}(x)\right|^{4}}{\left(\delta^{2}+\left|y_{\xi}(x)\right|^{2}\right)^{2}} d v_{g} .
$$

Since $\hat{U}_{\delta, \xi}$ satisfies in $B_{2 r_{0}}(\xi)$

$$
-\Delta_{g} \hat{U}_{\delta, \xi}=-\left.e^{-\hat{\varphi}_{\xi}(y)} \Delta u_{\delta, 0}\right|_{y=y_{\xi}(x)}=e^{-\varphi_{\xi}} e^{U_{\delta, \xi}}
$$


in view of (19), by the equation of $H(x, \xi)$ now we have that

$$
\begin{aligned}
-\Delta_{g} \Psi_{\delta, \xi}= & 2\left\langle\nabla \chi_{\xi}, \nabla \hat{U}_{\delta, \xi}+4 \nabla \log \left|y_{\xi}(x)\right|\right\rangle_{g}+\Delta_{g} \chi_{\xi}\left(\hat{U}_{\delta, \xi}+4 \log \left|y_{\xi}(x)\right|\right) \\
& +\frac{1}{|S|}\left(8 \pi-\int_{S} \chi_{\xi} e^{-\varphi_{\xi}} e^{U_{\delta, \xi}} d v_{g}\right) .
\end{aligned}
$$

Also, we have that in $A_{2 r_{0}}(\xi)$

$$
\hat{U}_{\delta, \xi}+4 \log \left|y_{\xi}(x)\right|=\log \frac{\left|y_{\xi}(x)\right|^{4}}{\left(\delta^{2}+\left|y_{\xi}(x)\right|^{2}\right)^{2}}=-2 \frac{\delta^{2}}{\left|y_{\xi}(x)\right|^{2}}+O\left(\delta^{4}\right)
$$

and

$$
\nabla\left(\hat{U}_{\delta, \xi}+4 \log \left|y_{\xi}(x)\right|\right)=-2 \delta^{2} \nabla\left|y_{\xi}(x)\right|^{-2}+O\left(\delta^{4}\right),
$$

and there holds

$$
\begin{aligned}
\int_{S} \chi_{\xi} e^{-\varphi_{\xi}} e^{U_{\delta, \xi}} d v_{g} & =\int_{B_{2 r_{0}}(0) \backslash B_{r_{0}}(0)} \chi(|y|) \frac{8 \delta^{2}}{|y|^{4}} d y+O\left(\delta^{4}\right)+\int_{B_{r_{0}}(0)} \frac{8 \delta^{2}}{\left(\delta^{2}+|y|^{2}\right)^{2}} d y \\
& =8 \pi-8 \delta^{2}\left(\frac{\pi}{r_{0}^{2}}-\int_{B_{2 r_{0}(0) \backslash B_{r_{0}}(0)}} \frac{\chi(|y|)}{|y|^{4}} d y\right)+O\left(\delta^{4}\right) \\
& =8 \pi+4 \delta^{2} \int_{\mathbb{R}^{2}} \frac{\chi^{\prime}(|y|)}{|y|^{3}} d y+O\left(\delta^{4}\right)
\end{aligned}
$$

in view of $d v_{g}=e^{\hat{\varphi} \xi} d y$ in the coordinate system $y_{\xi}$ and

$$
\int_{B_{2 r_{0}}(0) \backslash B_{r_{0}}(0)} \frac{\chi(|y|)}{|y|^{4}} d y=2 \pi \int_{r_{0}}^{2 r_{0}} \frac{\chi(r)}{r^{3}} d r=\frac{\pi}{r_{0}^{2}}+\frac{1}{2} \int_{B_{2 r_{0}}(0) \backslash B_{r_{0}}(0)} \frac{\chi^{\prime}(|y|)}{|y|^{3}} d y .
$$

By the definition of $f_{\xi}$ we then have that

$$
-\Delta_{g} \Psi_{\delta, \xi}=-2 \delta^{2} f_{\xi}+O\left(\delta^{4}\right) \quad \text { in } S .
$$

Since $\int_{S} F_{\xi} d v_{g}=0$, by elliptic regularity theory we get that

$$
\Psi_{\delta, \xi}=-2 \delta^{2} F_{\xi}+\frac{1}{|S|} \int_{S} \Psi_{\delta, \xi} d v_{g}+O\left(\delta^{4}\right),
$$

in view of (20). On the other hand, we have that

$$
\begin{aligned}
\int_{S} \Psi_{\delta, \xi} d v_{g}= & -\int_{S} \chi_{\xi} \log \frac{\left|y_{\xi}(x)\right|^{4}}{\left(\delta^{2}+\left|y_{\xi}(x)\right|^{2}\right)^{2}} d v_{g}=2 \int_{B_{r_{0}}(0)} \log \frac{\delta^{2}+|y|^{2}}{|y|^{2}} e^{\hat{\varphi}_{\xi}(y)} d y \\
& +\int_{B_{2 r_{0}(0) \backslash B_{r_{0}}(0)} \chi(|y|)\left(2 \frac{\delta^{2}}{|y|^{2}}+O\left(\delta^{4}\right)\right) e^{\hat{\varphi}_{\xi}(y)} d y} \\
& =2 \int_{B_{r_{0}}(0)} \log \frac{\delta^{2}+|y|^{2}}{|y|^{2}} e^{\hat{\varphi}_{\xi}(y)} d y+2 \delta^{2} \int_{\mathbb{R}^{2} \backslash B_{r_{0}}(0)} \chi(|y|) \frac{e^{\hat{\varphi}_{\xi}(y)}-1}{|y|^{2}} d y \\
& -4 \pi \delta^{2} \log r_{0}-2 \delta^{2} \int_{\mathbb{R}^{2}} \frac{\chi^{\prime}(|y|) \log |y|}{|y|} d y+O\left(\delta^{4}\right)
\end{aligned}
$$

in view of

$$
\int_{B_{2 r}(0) \backslash B_{r}(0)} \frac{\chi(|y|)}{|y|^{2}} d y=2 \pi \int_{r}^{2 r} \frac{\chi(t)}{t} d t=-2 \pi \log r-\int_{B_{2 r}(0) \backslash B_{r}(0)} \frac{\chi^{\prime}(|y|) \log |y|}{|y|} d y
$$


for $r \leq r_{0}$. Since

$$
\begin{aligned}
\int_{B_{r_{0}}(0)} \log \frac{\delta^{2}+|y|^{2}}{|y|^{2}} d y= & \delta^{2} \int_{B_{r_{0} / \delta}(0)} \log \frac{1+|z|^{2}}{|z|^{2}} d z=2 \pi \delta^{2} \int_{0}^{\infty}\left[\log \frac{1+r^{2}}{r^{2}}-\frac{1}{r^{2}+1}\right] r d r \\
& +\pi \delta^{2} \log \left(\frac{r_{0}^{2}}{\delta^{2}}+1\right)+O\left(\delta^{4}\right)
\end{aligned}
$$

where $y=\delta z$, by $e^{\hat{\varphi}_{\xi}(y)}=1+O\left(|y|^{2}\right)$ we can write that

$$
\begin{aligned}
& 2 \int_{B_{r_{0}}(0)} \log \frac{\delta^{2}+|y|^{2}}{|y|^{2}} e^{\hat{\varphi}_{\xi}(y)} d y=2 \int_{B_{r_{0}}(0)} \log \left(\frac{\delta^{2}}{|y|^{2}}+1\right)\left(e^{\hat{\varphi}_{\xi}(y)}-1\right) d y-4 \pi \delta^{2} \log \delta \\
& +4 \pi \delta^{2}\left[\log r_{0}+\int_{0}^{\infty}\left(\log \frac{1+r^{2}}{r^{2}}-\frac{1}{r^{2}+1}\right) r d r\right]+O\left(\delta^{4}\right) \\
& =2 \delta^{2} \int_{B_{r_{0}}(0)} \frac{e^{\hat{\varphi}_{\xi}(y)}-1}{|y|^{2}} d y-4 \pi \delta^{2} \log \delta+4 \pi \delta^{2}\left[\log r_{0}+\int_{0}^{\infty}\left(\log \frac{1+r^{2}}{r^{2}}-\frac{1}{r^{2}+1}\right) r d r\right] \\
& +O\left(\delta^{4}|\log \delta|\right)
\end{aligned}
$$

by using that

$$
\begin{aligned}
& \int_{B_{r_{0}}(0)}\left[\log \left(\frac{\delta^{2}}{|y|^{2}}+1\right)-\frac{\delta^{2}}{|y|^{2}}\right]\left(e^{\hat{\varphi}_{\xi}(y)}-1\right) d y=O\left(\delta^{4} \int_{B_{\frac{r_{0}}{\delta}(0)}}\left|\log \left(\frac{1}{|y|^{2}}+1\right)-\frac{1}{|y|^{2}}\right||y|^{2} d y\right) \\
& =O\left(\delta^{4} \int_{B_{\frac{r_{0}}{\delta}}(0) \backslash B_{1}(0)}\left|\log \left(\frac{1}{|y|^{2}}+1\right)-\frac{1}{|y|^{2}}\right||y|^{2} d y\right)+O\left(\delta^{4}\right)=O\left(\delta^{4}|\log \delta|\right)
\end{aligned}
$$

in view of $\log \left(\frac{1}{|y|^{2}}+1\right)-\frac{1}{|y|^{2}}=O\left(\frac{1}{|y|^{4}}\right)$ as $|y| \rightarrow+\infty$. In conclusion, we get that

$\int_{S} \Psi_{\delta, \xi} d v_{g}=-4 \pi \delta^{2} \log \delta+2 \delta^{2}\left[\int_{\mathbb{R}^{2}} \chi(|y|) \frac{e^{\hat{\varphi} \xi(y)}-1}{|y|^{2}} d y+\pi-\int_{\mathbb{R}^{2}} \frac{\chi^{\prime}(|y|) \log |y|}{|y|} d y\right]+O\left(\delta^{4}|\log \delta|\right)$

in view of $\int_{0}^{\infty}\left(\log \frac{1+r^{2}}{r^{2}}-\frac{1}{r^{2}+1}\right) r d r=\frac{1}{2}$. This completes the proof.

The ansatz will be constructed as follows. Given $m \in \mathbb{N}$, let us consider distinct points $\xi_{j} \in \tilde{S}$ (i.e. $\xi_{j} \in S$ with $k\left(\xi_{j}\right)>0$ ) and $\delta_{j}>0, j=1, \ldots, m$. In order to have a good approximation, we will assume that

$$
\delta_{j}^{2}=\delta^{2} \rho_{j}\left(\xi_{j}\right) \quad \forall j=1, \ldots, m,
$$

and

$$
\exists C>1:|\lambda-8 \pi m| \leq C \delta^{2}|\log \delta|,
$$

where $\delta>0$ and $\rho_{j}$ is as in (6). Up to take $r_{0}$ smaller, we assume that the points $\xi_{j}$ 's are well separated and $k\left(\xi_{j}\right)$ is uniformly far from zero, namely, we choose $\xi=\left(\xi_{1}, \ldots, \xi_{m}\right) \in \Xi$, where

$$
\Xi=\left\{\left(\xi_{1}, \ldots, \xi_{m}\right) \in S^{m} \mid d_{g}\left(\xi_{i}, \xi_{j}\right) \geq 4 r_{0} \text { and } k\left(\xi_{j}\right) \geq r_{0} \forall i, j=1, \ldots, m, i \neq j\right\} .
$$

Denote $U_{j}:=U_{\delta_{j}, \xi_{j}}$ and $W_{j}=P U_{j}, j=1, \ldots, m$, where $P$ is the projection operator defined by (18). Thus, our approximating solution is $W(x)=\sum_{j=1}^{m} W_{j}(x)$, parametrized by $(\delta, \xi) \in(0, \infty) \times \Xi$. Notice that for $r_{0}$ small enough we have that $\mathcal{D} \subset \Xi \subset \tilde{S}^{m} \backslash \Delta$. We will look for a solution $u$ of (1) in the form $u=W+\phi$, for some small remainder term $\phi$. In terms of $\phi$, the problem (11) is equivalent to find $\phi \in \bar{H}$ so that

$$
L(\phi)=-[R+N(\phi)] \quad \text { in } S
$$


where the linear operator $L$ is defined as

$$
L(\phi)=\Delta_{g} \phi+\lambda \frac{k e^{W}}{\int_{S} k e^{W} d v_{g}}\left(\phi-\frac{\int_{S} k e^{W} \phi d v_{g}}{\int_{S} k e^{W} d v_{g}}\right),
$$

the nonlinear part $N$ is given by

$$
N(\phi)=\lambda\left(\frac{k e^{W+\phi}}{\int_{S} k e^{W+\phi} d v_{g}}-\frac{k e^{W} \phi}{\int_{S} k e^{W} d v_{g}}+\frac{k e^{W} \int_{S} k e^{W} \phi d v_{g}}{\left(\int_{S} k e^{W} d v_{g}\right)^{2}}-\frac{k e^{W}}{\int_{S} k e^{W} d v_{g}}\right)
$$

and the approximation rate of $W$ is encoded in

$$
R=\Delta_{g} W+\lambda\left(\frac{k e^{W}}{\int_{S} k e^{W} d v_{g}}-\frac{1}{|S|}\right) .
$$

Notice that for all $\phi \in \bar{H}$

$$
\int_{S} L(\phi) d v_{g}=\int_{S} N(\phi) d v_{g}=\int_{S} R d v_{g}=0 .
$$

In order to get the invertibility of $L$, let us introduce the weighted norm

$$
\|h\|_{*}=\sup _{x \in S}\left[\sum_{j=1}^{m} \frac{\delta_{j}^{\sigma}}{\left(\delta_{j}^{2}+\chi_{B_{r_{0}}\left(\xi_{j}\right)}(x)\left|y_{\xi_{j}}(x)\right|^{2}+r_{0}^{2} \chi_{S \backslash B_{r_{0}}\left(\xi_{j}\right)}(x)\right)^{1+\sigma / 2}}\right]^{-1}|h(x)|
$$

for any $h \in L^{\infty}(S)$, where $0<\sigma<1$ is a small fixed constant and $\chi_{A}$ denotes the characteristic function of the set $A$. Let us evaluate the approximation rate of $W$ in $\|\cdot\|_{*}$ :

Lemma 2.2. Assume (24)-(25). There exists a constant $C>0$, independent of $\delta>0$ small, such that

$$
\|R\|_{*} \leq C\left(\delta\left|\nabla \varphi_{m}(\xi)\right|_{g}+\delta^{2-\sigma}|\log \delta|\right)
$$

for all $\xi \in \Xi$, where $\left|\nabla \varphi_{m}(\xi)\right|_{g}^{2}$ stands for $\sum_{j=1}^{m}\left|\nabla_{\xi_{j}} \varphi_{m}(\xi)\right|_{g}^{2}$.

Proof: First, from Lemma 2.1 we note that for any $j \in\{1, \ldots, m\}$

$$
W_{j}(x)=U_{j}(x)-\log \left(8 \delta_{j}^{2}\right)+8 \pi H\left(x, \xi_{j}\right)+O\left(\delta^{2}|\log \delta|\right)
$$

uniformly for $x \in B_{r_{0}}\left(\xi_{j}\right)$ and

$$
W_{j}(x)=8 \pi G\left(x, \xi_{j}\right)+O\left(\delta^{2}|\log \delta|\right)
$$

uniformly for $x$ on compact subsets of $S \backslash\left\{\xi_{j}\right\}$. Since by symmetry and $\hat{\varphi}_{\xi_{j}}(0)=0$ we have

$$
\begin{aligned}
\int_{B_{r_{0}}\left(\xi_{j}\right)} \rho_{j}(x) e^{U_{j}} d v_{g} & =\int_{B_{r_{0}}(0)} \rho_{j}\left(y_{\xi_{j}}^{-1}(y)\right) \frac{8 \delta_{j}^{2}}{\left(\delta_{j}^{2}+|y|^{2}\right)^{2}} e^{\hat{\varphi}_{\xi_{j}}(y)} d y \\
& =\int_{B_{\frac{r_{0}}{\partial_{j}}(0)}} \rho_{j}\left(\xi_{j}\right) \frac{8}{\left(1+|y|^{2}\right)^{2}}\left(1+O\left(\delta_{j}^{2}|y|^{2}\right)\right) d y=8 \pi \rho_{j}\left(\xi_{j}\right)+O\left(\delta^{2}|\log \delta|\right),
\end{aligned}
$$

we then get that

$$
\begin{aligned}
\int_{S} k e^{W} d v_{g} & =\sum_{j=1}^{m} \frac{1}{8 \delta_{j}^{2}} \int_{B_{r_{0}}\left(\xi_{j}\right)} k e^{U_{j}+8 \pi H\left(x, \xi_{j}\right)+8 \pi \sum_{l \neq j} G\left(x, \xi_{l}\right)+O\left(\delta^{2}|\log \delta|\right)} d v_{g}+O(1) \\
& =\sum_{j=1}^{m} \frac{1}{8 \delta_{j}^{2}} \int_{B_{r_{0}}\left(\xi_{j}\right)} \rho_{j}(x) e^{U_{j}}\left(1+O\left(\delta^{2}|\log \delta|\right)\right) d v_{g}+O(1) \\
& =\sum_{j=1}^{m} \frac{1}{\delta_{j}^{2}}\left[\pi \rho_{j}\left(\xi_{j}\right)+O\left(\delta^{2}|\log \delta|\right)\right]+O(1)=\frac{\pi m}{\delta^{2}}+O(|\log \delta|) .
\end{aligned}
$$


By Lemma 2.1 and (24), (31) we have that

- in $S \backslash \cup_{j=1}^{m} B_{r_{0}}\left(\xi_{j}\right)$ there holds $8 \pi m \frac{k e^{W}}{\int_{S} k e^{W} d v_{g}}=O\left(\delta^{2}\right)$ in view of $W(x)=O(1)$;

- in $B_{r_{0}}\left(\xi_{j}\right), j \in\{1, \ldots, m\}$, there holds

$$
\begin{aligned}
8 \pi m \frac{k e^{W}}{\int_{S} k e^{W} d v_{g}} & =8 \pi m \frac{k e^{-\log \left(8 \delta_{j}^{2}\right)+8 \pi H\left(x, \xi_{j}\right)+8 \pi \sum_{l \neq j} G\left(x, \xi_{l}\right)+O\left(\delta^{2}|\log \delta|\right)}}{\pi m \delta^{-2}+O(|\log \delta|)} e^{U_{j}} \\
& =\frac{8 \pi m \rho_{j}(x)+O\left(\delta^{2}|\log \delta|\right)}{8 \pi m \rho_{j}\left(\xi_{j}\right)+O\left(\delta^{2}|\log \delta|\right)} e^{U_{j}} \\
& =\left[1+\left\langle\frac{\nabla\left(\rho_{j} \circ y_{\xi_{j}}^{-1}\right)(0)}{\rho_{j}\left(\xi_{j}\right)}, y_{\xi_{j}}(x)\right\rangle+O\left(\left|y_{\xi_{j}}(x)\right|^{2}+\delta^{2}|\log \delta|\right)\right] e^{U_{j}},
\end{aligned}
$$

which can be summarized as follows:

$$
\begin{aligned}
\frac{8 \pi m k e^{W}}{\int_{S} k e^{W} d v_{g}}= & \sum_{j=1}^{m} \chi_{j}\left[1+\left\langle\frac{\nabla\left(\rho_{j} \circ y_{\xi_{j}}^{-1}\right)(0)}{\rho_{j}\left(\xi_{j}\right)}, y_{\xi_{j}}(x)\right\rangle+O\left(\left|y_{\xi_{j}}(x)\right|^{2}+\delta^{2}|\log \delta|\right)\right] e^{U_{j}} \\
& +O\left(\delta^{2}\right) \chi_{S \backslash \cup_{j=1}^{m} B_{r_{0}}\left(\xi_{j}\right)}
\end{aligned}
$$

where $\chi_{j}=\chi_{\xi_{j}}$. Since as before

$$
\int_{S} \chi_{j} e^{-\varphi_{j}} e^{U_{j}} d v_{g}=\int_{B_{r_{0}}(0)} \frac{8 \delta_{j}^{2}}{\left(\delta_{j}^{2}+|y|^{2}\right)^{2}} d y+O\left(\delta^{2}\right)=8 \pi+O\left(\delta^{2}\right)
$$

with $\varphi_{j}=\varphi_{\xi_{j}}$, for

$$
R_{8 \pi m}=\Delta_{g} W+8 \pi m\left(\frac{k e^{W}}{\int_{S} k e^{W} d v_{g}}-\frac{1}{|S|}\right)
$$

we then have that

$$
\begin{aligned}
R_{8 \pi m} & =-\sum_{j=1}^{m} \chi_{j} e^{-\varphi_{j}} e^{U_{j}}+8 \pi m \frac{k e^{W}}{\int_{S} k e^{W} d v_{g}}+\frac{1}{|S|} \sum_{j=1}^{m} \int_{S} \chi_{j} e^{-\varphi_{j}} e^{U_{j}} d v_{g}-\frac{8 \pi m}{|S|} \\
= & -\sum_{j=1}^{m} \chi_{j} e^{-\varphi_{j}} e^{U_{j}}+8 \pi m \frac{k e^{W}}{\int_{S} k e^{W} d v_{g}}+O\left(\delta^{2}\right) .
\end{aligned}
$$

By (32) we now deduce that $R_{8 \pi m}(x)=O\left(\delta^{2}\right)$ in $S \backslash \cup_{j=1}^{m} B_{r_{0}}\left(\xi_{j}\right)$ and

$$
\begin{aligned}
R_{8 \pi m} & =\left[-e^{-\varphi_{j}}+1+O\left(\left|\nabla \log \left(\rho_{j} \circ y_{\xi_{j}}^{-1}\right)(0)\right|\left|y_{\xi_{j}}(x)\right|+\delta^{2}|\log \delta|\right)\right] e^{U_{j}}+O\left(\delta^{2}\right) \\
& =e^{U_{j}} O\left(\left|\nabla \log \left(\rho_{j} \circ y_{\xi_{j}}^{-1}\right)(0)\right|\left|y_{\xi_{j}}(x)\right|+\left|y_{\xi_{j}}(x)\right|^{2}+\delta^{2}|\log \delta|\right)+O\left(\delta^{2}\right)
\end{aligned}
$$

in $B_{r_{0}}\left(\xi_{j}\right), j \in\{1, \ldots, m\}$, in view of $\varphi_{j}\left(\xi_{j}\right)=0$ and $\nabla \varphi_{j}\left(\xi_{j}\right)=0$. From the definition of $\|\cdot\|_{*}$ we deduce the validity of

$$
\left\|R_{8 \pi m}\right\|_{*} \leq C\left(\delta\left|\nabla \varphi_{m}(\xi)\right|_{g}+\delta^{2-\sigma}\right)
$$

in view of $\left|\nabla \log \left(\rho_{j} \circ y_{\xi_{j}}^{-1}\right)(0)\right| \leq\left|\nabla_{\xi_{j}} \varphi_{m}(\xi)\right|_{g}$. Since by (32)

$$
R-R_{8 \pi m}=(\lambda-8 \pi m)\left(\frac{k e^{W}}{\int_{S} k e^{W} d v_{g}}-\frac{1}{|S|}\right)=O\left(|\lambda-8 \pi m| \sum_{j=1}^{m} \chi_{j} e^{U_{j}}+|\lambda-8 \pi m|\right),
$$

we get that $\left\|R-R_{8 \pi m}\right\|_{*}=O\left(\delta^{-\sigma}|\lambda-8 \pi m|\right)$. In conclusion, by (25) and (33) we deduce the validity of (30). 


\section{The reduced energy}

The purpose of this section is to give an asymptotic expansion of the "reduced energy" $J_{\lambda}(W)$, where $J_{\lambda}$ is the energy functional given by (4). For technical reasons, we will be concerned with establishing it in a $C^{2}$-sense in $\delta$ and just in a $C^{1}$-sense in $\xi$. To this aim, the following result will be very useful:

Lemma 3.1. Letting $f \in C^{2, \gamma}(S)$ (possibly depending in $\xi$ ), $0<\gamma<1$, denote as $P_{2}(f)$ the second-order Taylor expansion of $f(x)$ at $\xi$ :

$$
P_{2} f(x)=f(\xi)+\left\langle\nabla\left(f \circ y_{\xi}^{-1}\right)(0), y_{\xi}(x)\right\rangle+\frac{1}{2}\left\langle D^{2}\left(f \circ y_{\xi}^{-1}\right)(0) y_{\xi}(x), y_{\xi}(x)\right\rangle .
$$

The following expansions do hold as $\delta \rightarrow 0$ :

$$
\begin{aligned}
& \int_{S} \chi_{\xi} e^{-\varphi_{\xi}} f(x) e^{U_{\delta, \xi}} d v_{g}=8 \pi f(\xi)-2 \delta^{2} \Delta_{g} f(\xi)\left[2 \pi \log \delta+\int_{\mathbb{R}^{2}} \frac{\chi^{\prime}(|y|) \log |y|}{|y|} d y+\pi\right] \\
& +8 \delta^{2} \int_{S} \chi_{\xi} e^{-\varphi_{\xi}} \frac{f(x)-P_{2}(f)(x)}{\left|y_{\xi}(x)\right|^{4}} d v_{g}+4 \delta^{2} f(\xi) \int_{\mathbb{R}^{2}} \frac{\chi^{\prime}(|y|)}{|y|^{3}} d y+o\left(\delta^{2}\right), \\
& \int_{S} \chi_{\xi} e^{-\varphi_{\xi}} f(x) e^{U_{\delta, \xi}} \frac{d v_{g}}{\delta^{2}+\left|y_{\xi}(x)\right|^{2}}=\frac{4 \pi}{\delta^{2}} f(\xi)+\pi \Delta_{g} f(\xi)+O\left(\delta^{\gamma}\right)
\end{aligned}
$$

and

$$
\int_{S} \chi_{\xi} e^{-\varphi_{\xi}} f(x) e^{U_{\delta, \xi}} \frac{a \delta^{2}-\left|y_{\xi}(x)\right|^{2}}{\left(\delta^{2}+\left|y_{\xi}(x)\right|^{2}\right)^{2}} d v_{g}=\frac{4 \pi}{3 \delta^{2}}(2 a-1) f(\xi)+(a-2) \frac{\pi}{3} \Delta_{g} f(\xi)+O\left(\delta^{\gamma}\right)
$$

for $a \in \mathbb{R}$.

Proof: Since $d v_{g}=e^{\hat{\varphi}_{\xi}(y)} d y$, by symmetry observe that

$$
\begin{aligned}
& \int_{S \backslash B_{r_{0}}(\xi)} \chi_{\xi} e^{-\varphi_{\xi}} f(x) e^{U_{\delta, \xi}} d v_{g}=8 \delta^{2} \int_{S \backslash B_{r_{0}}(\xi)} \frac{\chi_{\xi} e^{-\varphi_{\xi}} f(x)}{\left|y_{\xi}(x)\right|^{4}} d v_{g}+O\left(\delta^{4}\right) \\
& =8 \delta^{2} \int_{S \backslash B_{r_{0}}(\xi)} \chi_{\xi} e^{-\varphi_{\xi}} \frac{f(x)-P_{2}(f)(x)}{\left|y_{\xi}(x)\right|^{4}} d v_{g}+8 \delta^{2} f(\xi) \int_{B_{2 r_{0}}(0) \backslash B_{r_{0}}(0)} \frac{\chi(|y|)}{|y|^{4}} d y \\
& +2 \delta^{2} \Delta\left(f \circ y_{\xi}^{-1}\right)(0) \int_{B_{2 r_{0}}(0) \backslash B_{r_{0}}(0)} \frac{\chi(|y|)}{|y|^{2}} d y+O\left(\delta^{4}\right)
\end{aligned}
$$

as $\delta \rightarrow 0$. On $B_{r_{0}}(\xi)$ we get that

$$
\begin{aligned}
& \int_{B_{r_{0}}(\xi)} \chi \xi e^{-\varphi_{\xi}} f(x) e^{U_{\delta, \xi}} d v_{g}=\int_{B_{r_{0}}(0)} f\left(y_{\xi}^{-1}(y)\right) \frac{8 \delta^{2}}{\left(\delta^{2}+|y|^{2}\right)^{2}} d y \\
& =\int_{B_{r_{0}}(0)} P_{2}(f)\left(y_{\xi}^{-1}(y)\right) \frac{8 \delta^{2}}{\left(\delta^{2}+|y|^{2}\right)^{2}} d y+\int_{B_{r_{0}}(0)}\left(\left(f \circ y_{\xi}^{-1}\right)(y)-P_{2}(f)\left(y_{\xi}^{-1}(y)\right)\right) \frac{8 \delta^{2}}{\left(\delta^{2}+|y|^{2}\right)^{2}} d y
\end{aligned}
$$


Since $f(x)-P_{2}(f)(x)=O\left(\left|y_{\xi}(x)\right|^{2+\gamma}\right)$, by symmetry and the Lebesgue Theorem we get that

$$
\begin{aligned}
& \int_{B_{r_{0}}(\xi)} \chi_{\xi} e^{-\varphi_{\xi}} f(x) e^{U_{\delta, \xi}} d v_{g}=f(\xi) \int_{B_{r_{0} / \delta}(0)} \frac{8}{\left(1+|y|^{2}\right)^{2}} d y \\
& +\delta^{2} \Delta\left(f \circ y_{\xi}^{-1}\right)(0) \int_{B_{r_{0} / \delta}(0)} \frac{2|y|^{2}}{\left(1+|y|^{2}\right)^{2}} d y+8 \delta^{2} \int_{B_{r_{0}}(\xi)} e^{-\varphi_{\xi}} \frac{f(x)-P_{2}(f)(x)}{\left|y_{\xi}(x)\right|^{4}} d v_{g}+o\left(\delta^{2}\right) \\
& =8 \pi f(\xi)\left(1-\frac{\delta^{2}}{\delta^{2}+r_{0}^{2}}\right)+2 \pi \delta^{2} \Delta\left(f \circ y_{\xi}^{-1}\right)(0)\left(\log \frac{\delta^{2}+r_{0}^{2}}{\delta^{2}}+\frac{\delta^{2}}{\delta^{2}+r_{0}^{2}}-1\right) \\
& +8 \delta^{2} \int_{B_{r_{0}}(\xi)} e^{-\varphi_{\xi}} \frac{f(x)-P_{2}(f)(x)}{\left|y_{\xi}(x)\right|^{4}} d v_{g}+o\left(\delta^{2}\right)=8 \pi f(\xi)\left(1-\frac{\delta^{2}}{r_{0}^{2}}\right) \\
& +2 \pi \delta^{2}\left(-2 \log \delta+2 \log r_{0}-1\right) \Delta\left(f \circ y_{\xi}^{-1}\right)(0)+8 \delta^{2} \int_{B_{r_{0}}(\xi)} e^{-\varphi_{\xi}} \frac{f(x)-P_{2}(f)(x)}{\left|y_{\xi}(x)\right|^{4}} d v_{g}+o\left(\delta^{2}\right) .
\end{aligned}
$$

In view of (23) and

$$
\int_{B_{2 r}(0) \backslash B_{r}(0)} \frac{\chi(|y|)}{|y|^{4}} d y=2 \pi \int_{r}^{2 r} \frac{\chi(t)}{t^{3}} d t=\frac{\pi}{r^{2}}+\frac{1}{2} \int_{B_{2 r}(0) \backslash B_{r}(0)} \frac{\chi^{\prime}(|y|)}{|y|^{3}} d y
$$

for $r \leq r_{0}$, summing up the two previous expansions we get that

$$
\begin{aligned}
\int_{S} \chi_{\xi} e^{-\varphi_{\xi}} f(x) e^{U_{\delta, \xi}} d v_{g}= & 8 \pi f(\xi)-2 \delta^{2} \Delta\left(f \circ y_{\xi}^{-1}\right)(0)\left[2 \pi \log \delta+\int_{\mathbb{R}^{2}} \frac{\chi^{\prime}(|y|) \log |y|}{|y|} d y+\pi\right] \\
& +8 \delta^{2} \int_{S} \chi_{\xi} e^{-\varphi_{\xi}} \frac{f(x)-P_{2}(f)(x)}{\left|y_{\xi}(x)\right|^{4}} d v_{g}+4 \delta^{2} f(\xi) \int_{\mathbb{R}^{2}} \frac{\chi^{\prime}(|y|)}{|y|^{3}} d y+o\left(\delta^{2}\right) .
\end{aligned}
$$

Since by (19) $\Delta_{g} f(x)=e^{-\varphi_{\xi}(x)} \Delta\left(f \circ y_{\xi}^{-1}\right)\left(y_{\xi}(x)\right)$, we get that $\Delta\left(f \circ y_{\xi}^{-1}\right)(0)=\Delta_{g} f(\xi)$, and the validity of the first expansion then follows. The other two expansions are simpler because of the stronger decay. Indeed, by the Taylor expansion of $f$ at $\xi$ and the symmetries we get that

$$
\begin{aligned}
& \int_{S} \chi_{\xi} e^{-\varphi_{\xi}} f(x) e^{U_{\delta, \xi}} \frac{d v_{g}}{\delta^{2}+\left|y_{\xi}(x)\right|^{2}}=\frac{8}{\delta^{2}} \int_{B_{r_{0} / \delta}(0)}\left(f \circ y_{\xi}^{-1}\right)(\delta y) \frac{d y}{\left(1+|y|^{2}\right)^{3}}+O\left(\delta^{2}\right) \\
& =\frac{8}{\delta^{2}}\left[f(\xi) \int_{\mathbb{R}^{2}} \frac{d y}{\left(1+|y|^{2}\right)^{3}}+\frac{\delta^{2}}{4} \Delta_{g} f(\xi) \int_{\mathbb{R}^{2}} \frac{|y|^{2}}{\left(1+|y|^{2}\right)^{3}} d y+O\left(\delta^{2+\gamma} \int_{\mathbb{R}^{2}} \frac{|y|^{2+\gamma}}{\left(1+|y|^{2}\right)^{3}} d y\right)\right]+O\left(\delta^{2}\right) \\
& =\frac{4 \pi}{\delta^{2}} f(\xi)+\pi \Delta_{g} f(\xi)+O\left(\delta^{\gamma}\right)
\end{aligned}
$$

in view of $\int_{\mathbb{R}^{2}} \frac{d y}{\left(1+|y|^{2}\right)^{3}}=\frac{\pi}{2}$ and

$$
\int_{\mathbb{R}^{2}} \frac{|y|^{2}}{\left(1+|y|^{2}\right)^{3}} d y=\int_{\mathbb{R}^{2}} \frac{d y}{\left(1+|y|^{2}\right)^{2}}-\int_{\mathbb{R}^{2}} \frac{d y}{\left(1+|y|^{2}\right)^{3}}=\frac{\pi}{2} .
$$

Similarly, we have that

$$
\begin{aligned}
& \int_{S} \chi_{\xi} e^{-\varphi_{\xi}} f(x) e^{U_{\delta, \xi}} \frac{a \delta^{2}-\left|y_{\xi}(x)\right|^{2}}{\left(\delta^{2}+\left|y_{\xi}(x)\right|^{2}\right)^{2}} d v_{g}=\frac{8}{\delta^{2}} \int_{B_{r_{0} / \delta}(0)}\left(f \circ y_{\xi}^{-1}\right)(\delta y) \frac{a-|y|^{2}}{\left(1+|y|^{2}\right)^{4}} d y+O\left(\delta^{2}\right) \\
& =\frac{8}{\delta^{2}}\left[f(\xi) \int_{\mathbb{R}^{2}} \frac{a-|y|^{2}}{\left(1+|y|^{2}\right)^{4}} d y+\frac{\delta^{2}}{4} \Delta_{g} f(\xi) \int_{\mathbb{R}^{2}} \frac{|y|^{2}\left(a-|y|^{2}\right)}{\left(1+|y|^{2}\right)^{4}} d y\right]+O\left(\delta^{\gamma}\right) \\
& =\frac{4 \pi}{3 \delta^{2}}(2 a-1) f(\xi)+(a-2) \frac{\pi}{3} \Delta_{g} f(\xi)+O\left(\delta^{\gamma}\right)
\end{aligned}
$$

in view of

$$
\int_{\mathbb{R}^{2}} \frac{a-|y|^{2}}{\left(1+|y|^{2}\right)^{4}} d y=(a+1) \int_{\mathbb{R}^{2}} \frac{d y}{\left(1+|y|^{2}\right)^{4}}-\int_{\mathbb{R}^{2}} \frac{d y}{\left(1+|y|^{2}\right)^{3}}=(2 a-1) \frac{\pi}{6}
$$


and

$\int_{\mathbb{R}^{2}} \frac{|y|^{2}\left(a-|y|^{2}\right)}{\left(1+|y|^{2}\right)^{4}} d y=-\int_{\mathbb{R}^{2}} \frac{d y}{\left(1+|y|^{2}\right)^{2}}+(2+a) \int_{\mathbb{R}^{2}} \frac{d y}{\left(1+|y|^{2}\right)^{3}}-(1+a) \int_{\mathbb{R}^{2}} \frac{d y}{\left(1+|y|^{2}\right)^{4}}=(a-2) \frac{\pi}{6}$.

The Lemma is completely established.

We are now ready to establish the expansion of $J_{\lambda}(W)$ :

Theorem 3.2. Assume (24)-(25). The following expansion does hold

$$
J_{\lambda}(W)=-8 \pi m-\lambda \log (\pi m)-32 \pi^{2} \varphi_{m}(\xi)+2(\lambda-8 \pi m) \log \delta+A(\xi) \delta^{2} \log \delta-B(\xi) \delta^{2}+o\left(\delta^{2}\right)
$$

in $C^{2}(\mathbb{R})$ and $C^{1}(\Xi)$ as $\delta \rightarrow 0^{+}$, where $\varphi_{m}(\xi), A(\xi)$ and $B(\xi)$ are given by (5), (7) and (12), respectively.

The proof will be divided into several steps.

Proof (of (35) in $C(\mathbb{R} \times \Xi)$ ): First, let us consider the term

$$
\int_{S}|\nabla W|_{g}^{2} d v_{g}=\int_{S} W\left(-\Delta_{g} W\right) d v_{g}=\sum_{j, l=1}^{m} \int_{S} \chi_{j} e^{-\varphi_{j}} e^{U_{j}} W_{l} d v_{g}
$$

in view of $\int_{S} W d v_{g}=0$. Since by (3) and (18)

$$
\int_{S} \chi_{j} e^{-\varphi_{j}} e^{U_{j}} G\left(x, \xi_{l}\right) d v_{g}=\int_{S}\left(-\Delta_{g} P U_{j}\right) G\left(x, \xi_{l}\right) d v_{g}=P U_{j}\left(\xi_{l}\right)
$$

for all $j, l=1, \ldots, m$, by Lemmata 2.1, 3.1 and (36) we have that for $l=j$

$$
\begin{aligned}
& \int_{S} \chi_{j} e^{-\varphi_{j}} e^{U_{j}} W_{j} d v_{g} \\
& =\int_{S} \chi_{j} e^{-\varphi_{j}} e^{U_{j}}\left[\chi_{j}\left(U_{j}-\log \left(8 \delta_{j}^{2}\right)\right)+8 \pi H\left(x, \xi_{j}\right)+\alpha_{\delta_{j}, \xi_{j}}-2 \delta_{j}^{2} F_{\xi_{j}}\right] d v_{g}+O\left(\delta^{4}|\log \delta|\right) \\
& =\int_{S} \chi_{j} e^{-\varphi_{j}} e^{U_{j}}\left[\chi_{j} \log \frac{\left|y_{\xi_{j}}(x)\right|^{4}}{\left(\delta_{j}^{2}+\left|y_{\xi_{j}}(x)\right|^{2}\right)^{2}}+8 \pi G\left(x, \xi_{j}\right)+\alpha_{\delta_{j}, \xi_{j}}-2 \delta_{j}^{2} F_{\xi_{j}}\right] d v_{g}+O\left(\delta^{4}|\log \delta|\right) \\
& =8 \int_{B_{2 r_{0} / \delta_{j}}(0)} \frac{\chi^{2}\left(\delta_{j}|y|\right)}{\left(1+|y|^{2}\right)^{2}} \log \frac{|y|^{4}}{\left(1+|y|^{2}\right)^{2}} d y+8 \pi P U_{j}\left(\xi_{j}\right)+8 \pi \alpha_{\delta_{j}, \xi_{j}}-16 \pi \delta_{j}^{2} F_{\xi_{j}}\left(\xi_{j}\right) \\
& +O\left(\delta^{4}|\log \delta|^{2}\right)=-16 \pi-32 \pi \log \delta_{j}+64 \pi^{2} H\left(\xi_{j}, \xi_{j}\right)+16 \pi \alpha_{\delta_{j}, \xi_{j}}-32 \pi \delta_{j}^{2} F_{\xi_{j}}\left(\xi_{j}\right)+O\left(\delta^{4}|\log \delta|^{2}\right)
\end{aligned}
$$

in view of

$$
\int_{\mathbb{R}^{2}} \frac{d y}{\left(1+|y|^{2}\right)^{2}} \log \frac{|y|^{4}}{\left(1+|y|^{2}\right)^{2}}=2 \pi \int_{0}^{\infty} \frac{d s}{(1+s)^{2}} \log \frac{s}{1+s}=-2 \pi \int_{0}^{\infty} \frac{d s}{(1+s)^{2}}=-2 \pi
$$

by means of an integration by parts. Similarly, by Lemmata 2.1, 3.1 and (36) we have that for $l \neq j$

$$
\begin{aligned}
& \int_{S} \chi_{j} e^{-\varphi_{j}} e^{U_{j}} W_{l} d v_{g}=\int_{S} \chi_{j} e^{-\varphi_{j}} e^{U_{j}}\left[8 \pi G\left(x, \xi_{l}\right)+\alpha_{\delta_{l}, \xi_{l}}-2 \delta_{l}^{2} F_{\xi_{l}}\right] d v_{g}+O\left(\delta^{4}|\log \delta|\right) \\
& =64 \pi^{2} G\left(\xi_{l}, \xi_{j}\right)+8 \pi\left(\alpha_{\delta_{j}, \xi_{j}}+\alpha_{\delta_{l}, \xi_{l}}\right)-16 \pi\left(\delta_{j}^{2} F_{\xi_{j}}\left(\xi_{l}\right)+\delta_{l}^{2} F_{\xi_{l}}\left(\xi_{j}\right)\right)+O\left(\delta^{4}|\log \delta|^{2}\right) .
\end{aligned}
$$

Setting

$$
\alpha_{\delta, \xi}=\sum_{j=1}^{m} \alpha_{\delta_{j}, \xi_{j}} \quad F_{\delta, \xi}(x)=\sum_{j=1}^{m} \delta_{j}^{2} F_{\xi_{j}}(x),
$$


summing up the two previous expansions, for the gradient term we get that

$$
\frac{1}{2} \int_{S}|\nabla W|_{g}^{2} d v_{g}=-8 \pi m-16 \pi m \log \delta-32 \pi^{2} \varphi_{m}(\xi)+8 \pi m \alpha_{\delta, \xi}-16 \pi \sum_{j=1}^{m} F_{\delta, \xi}\left(\xi_{j}\right)+o\left(\delta^{2}\right)
$$

in view of (24) and

$$
8 \pi \sum_{j=1}^{m} \log \rho_{j}\left(\xi_{j}\right)-32 \pi^{2}\left[\sum_{j=1}^{m} H\left(\xi_{j}, \xi_{j}\right)+\sum_{l \neq j} G\left(\xi_{l}, \xi_{j}\right)\right]=32 \pi^{2} \varphi_{m}(\xi) .
$$

Let us now expand the potential term in $J_{\lambda}(W)$. By Lemma 2.1 for any $j=1, \ldots, m$ we find that

$$
\begin{aligned}
\int_{B_{r_{0}}\left(\xi_{j}\right)} k e^{W} d v_{g} & =\int_{B_{r_{0}}\left(\xi_{j}\right)} \rho_{j} e^{U_{j}-\log \left(8 \delta_{j}^{2}\right)+\alpha_{\delta, \xi}-2 F_{\delta, \xi}+O\left(\delta^{4}|\log \delta|\right)} d v_{g} \\
& =\frac{1}{8 \delta_{j}^{2}}\left[\int_{S} \chi_{j} e^{U_{j}} \rho_{j} e^{\alpha_{\delta, \xi}-2 F_{\delta, \xi}} d v_{g}-8 \delta_{j}^{2} \int_{A_{2 r_{0}}\left(\xi_{j}\right)} \frac{\chi_{j} \rho_{j}}{\left|y_{\xi_{j}}(x)\right|^{4}} d v_{g}+O\left(\delta^{4}|\log \delta|\right)\right] .
\end{aligned}
$$

By Lemma 3.1 (with $f(x)=e^{\varphi_{j}} \rho_{j} e^{\alpha_{\delta, \xi}-2 F_{\delta, \xi}}$ ) we can now deduce that

$$
\begin{aligned}
& 8 \delta_{j}^{2} \int_{B_{r_{0}}\left(\xi_{j}\right)} k e^{W} d v_{g}=8 \pi \rho_{j}\left(\xi_{j}\right) e^{\alpha_{\delta, \xi}-2 F_{\delta, \xi}\left(\xi_{j}\right)}-4 \pi\left(\Delta_{g} \rho_{j}\left(\xi_{j}\right)-2 K\left(\xi_{j}\right) \rho_{j}\left(\xi_{j}\right)\right) \delta_{j}^{2} \log \delta_{j} \\
& -2\left(\Delta_{g} \rho_{j}\left(\xi_{j}\right)-2 K\left(\xi_{j}\right) \rho_{j}\left(\xi_{j}\right)\right)\left(\int_{\mathbb{R}^{2}} \frac{\chi^{\prime}(|y|) \log |y|}{|y|} d y+\pi\right) \delta_{j}^{2}+4 \delta_{j}^{2} \rho_{j}\left(\xi_{j}\right) \int_{\mathbb{R}^{2}} \frac{\chi^{\prime}(|y|)}{|y|^{3}} d y \\
& +8 \delta_{j}^{2} \int_{B_{r_{0}}\left(\xi_{j}\right)}\left[k e^{8 \pi \sum_{j=1}^{m} G\left(x, \xi_{j}\right)}-e^{-\varphi_{j}} \frac{P_{2}\left(e^{\varphi_{j}} \rho_{j}\right)}{\left|y_{\xi_{j}}(x)\right|^{4}}\right] d v_{g}-8 \delta_{j}^{2} \int_{A_{2_{0}(}\left(\xi_{j}\right)} \chi_{j} e^{-\varphi_{j}} \frac{P_{2}\left(e^{\varphi_{j}} \rho_{j}\right)}{\left|y_{\xi_{j}}(x)\right|^{4}} d v_{g}+o\left(\delta^{2}\right)
\end{aligned}
$$

in view of $\frac{\rho_{j}}{\left|y_{\xi_{j}}(x)\right|^{4}}=k e^{8 \pi \sum_{j=1}^{m} G\left(x, \xi_{j}\right)}$ in $B_{r_{0}}\left(\xi_{j}\right)$ and by (17)

$$
\Delta_{g}\left[e^{\varphi_{j}} \rho_{j}\right]\left(\xi_{j}\right)=\Delta_{g} \rho_{j}\left(\xi_{j}\right)-2 K\left(\xi_{j}\right) \rho_{j}\left(\xi_{j}\right) .
$$

On the other hand, we have that

$$
\int_{S \backslash \cup_{j=1}^{m} B_{r_{0}}\left(\xi_{j}\right)} k e^{W} d v_{g}=\int_{S \backslash \cup_{j=1}^{m} B_{r_{0}}\left(\xi_{j}\right)} k e^{8 \pi \sum_{j=1}^{m} G\left(x, \xi_{j}\right)} d v_{g}+O\left(\delta^{2}|\log \delta|\right) .
$$

Since

$$
\sum_{j=1}^{m} e^{-2 F_{\delta, \xi}\left(\xi_{j}\right)}=m-2 \sum_{j=1}^{m} F_{\delta, \xi}\left(\xi_{j}\right)+O\left(\delta^{4}\right)
$$

and by (24) there holds

$$
\delta_{j}^{2} \log \delta_{j}=\rho_{j}\left(\xi_{j}\right) \delta^{2} \log \delta+\frac{1}{2} \rho_{j}\left(\xi_{j}\right) \log \rho_{j}\left(\xi_{j}\right) \delta^{2},
$$

we then obtain that

$$
\frac{1}{\pi} e^{-\alpha_{\delta, \xi}} \delta^{2} \int_{S} k e^{W} d v_{g}=m-\frac{A(\xi)}{8 \pi} \delta^{2} \log \delta+\frac{B_{\chi}(\xi)}{8 \pi} \delta^{2}-2 \sum_{j=1}^{m} F_{\delta, \xi}\left(\xi_{j}\right)+o\left(\delta^{2}\right),
$$

where

$$
\begin{aligned}
B_{\chi}(\xi)= & -2 \pi \sum_{j=1}^{m}\left[\Delta_{g} \rho_{j}\left(\xi_{j}\right)-2 K\left(\xi_{j}\right) \rho_{j}\left(\xi_{j}\right)\right] \log \rho_{j}\left(\xi_{j}\right)-\frac{A(\xi)}{2 \pi}\left(\int_{\mathbb{R}^{2}} \frac{\chi^{\prime}(|y|) \log |y|}{|y|} d y+\pi\right) \\
& +4 \int_{\mathbb{R}^{2}} \frac{\chi^{\prime}(|y|)}{|y|^{3}} d y \sum_{j=1}^{m} \rho_{j}\left(\xi_{j}\right)+8 \int_{S}\left[k e^{8 \pi \sum_{j=1}^{m} G\left(x, \xi_{j}\right)}-\sum_{j=1}^{m} \chi_{j} e^{-\varphi_{j}} \frac{P_{2}\left(e^{\varphi_{j}} \rho_{j}\right)}{\left|y_{\xi_{j}}(x)\right|^{4}}\right] d v_{g} .
\end{aligned}
$$


By (23), (34) and the splitting of $S$ as the union of $\cup_{j=1}^{m} B_{r}\left(\xi_{j}\right)$ and $S \backslash \cup_{j=1}^{m} B_{r}\left(\xi_{j}\right), r \leq r_{0}$, we easily deduce that

$$
\begin{aligned}
B_{\chi}(\xi)= & -2 \pi \sum_{j=1}^{m}\left[\Delta_{g} \rho_{j}\left(\xi_{j}\right)-2 K\left(\xi_{j}\right) \rho_{j}\left(\xi_{j}\right)\right] \log \rho_{j}\left(\xi_{j}\right)-\frac{A(\xi)}{2} \\
& +8 \int_{S \backslash \cup_{j=1}^{m} B_{r}\left(\xi_{j}\right)} k e^{8 \pi \sum_{j=1}^{m} G\left(x, \xi_{j}\right)} d v_{g}-\frac{8 \pi}{r^{2}} \sum_{j=1}^{m} \rho_{j}\left(\xi_{j}\right)-A(\xi) \log \frac{1}{r} \\
& +8 \sum_{j=1}^{m} \int_{B_{r}\left(\xi_{j}\right)} \frac{e^{\varphi_{j}(x)} \rho_{j}(x)-P_{2}\left(e^{\varphi_{j}} \rho_{j}\right)(x)}{\left|y_{\xi_{j}}(x)\right|^{4}} e^{-\varphi_{j}(x)} d v_{g}
\end{aligned}
$$

in view of $\frac{\rho_{j}}{\left|y_{\xi_{j}}(x)\right|^{4}}=k e^{8 \pi \sum_{j=1}^{m} G\left(x, \xi_{j}\right)}$ in $B_{r_{0}}\left(\xi_{j}\right)$, (37) and the definitions of $A(\xi), P_{2}\left(e^{\varphi_{j}} \rho_{j}\right)$. As a by-product we have that $B_{\chi}(\xi)$ does not depend on $\chi$ and $r \leq r_{0}$. Since

$$
\lim _{r \rightarrow 0} \int_{B_{r}\left(\xi_{j}\right)} \frac{e^{\varphi_{j}(x)} \rho_{j}(x)-P_{2}\left(e^{\varphi_{j}} \rho_{j}\right)(x)}{\left|y_{\xi_{j}}(x)\right|^{4}} e^{-\varphi_{j}(x)} d v_{g}=0
$$

in view of $e^{\varphi_{j}(x)} \rho_{j}(x)-P_{2}\left(e^{\varphi_{j}} \rho_{j}\right)(x)=o\left(\left|y_{\xi_{j}}(x)\right|^{2}\right)$ as $x \rightarrow \xi_{j}$, we have that $B_{\chi}(\xi)$ coincides with $B(\xi)$ as defined in (12).

Finally, we get the following expansion for $J_{8 \pi m}(W)$ as $\delta \rightarrow 0$ :

$$
J_{8 \pi m}(W)=-8 \pi m(1+\log (\pi m))-32 \pi^{2} \varphi_{m}(\xi)+A(\xi) \delta^{2} \log \delta-B(\xi) \delta^{2}+o\left(\delta^{2}\right) .
$$

Since

$$
\log \int_{S} k e^{W} d v_{g}=-2 \log \delta+\log (\pi m)+O\left(\delta^{2}|\log \delta|\right),
$$

by (25) we then deduce that

$$
\begin{aligned}
J_{\lambda}(W) & =J_{8 \pi m}(W)-(\lambda-8 \pi m) \log \int_{S} k e^{W} d v_{g} \\
& =J_{8 \pi m}(W)-(\lambda-8 \pi m)(-2 \log \delta+\log (\pi m))+O\left(\delta^{4}|\log \delta|^{2}\right)
\end{aligned}
$$

and the proof is complete.

We establish now expansion (35) in a $C^{1}$-sense in $\xi$, where the derivatives in $\xi$ are with respect to a given coordinate system.

Proof (of (35) in $C^{1}(\Xi)$ ): We just need to expand the derivatives of $J_{\lambda}(W)$ in $\xi$. Let us fix $i \in\{1,2\}$ and $j \in\{1, \ldots, m\}$. We have that

$$
\partial_{\left(\xi_{j}\right)_{i}}\left[J_{\lambda}(W)\right]=-\int_{S}\left[\Delta_{g} W+\frac{\lambda k e^{W}}{\int_{S} k e^{W} d v_{g}}\right] \partial_{\left(\xi_{j}\right)_{i}} W d v_{g} .
$$

Arguing as in Lemma 2.1, it is easy to show that

$$
\begin{aligned}
\partial_{\left(\xi_{j}\right)_{i}} W_{q}= & -2 \frac{\chi_{q}}{\delta_{q}^{2}+\left|y_{\xi_{q}}(x)\right|^{2}}\left[\partial_{\left(\xi_{j}\right)_{i}}\left|y_{\xi_{q}}(x)\right|^{2}+\delta_{q}^{2} \partial_{\left(\xi_{j}\right)_{i}}\left(\log \rho_{q}\left(\xi_{q}\right)\right)\right] \\
& -4 \log \left|y_{\xi_{q}}(x)\right| \partial_{\left(\xi_{j}\right)_{i}} \chi_{q}+8 \pi \partial_{\left(\xi_{j}\right)_{i}} H\left(x, \xi_{q}\right)+O\left(\delta^{2}|\log \delta|\right)
\end{aligned}
$$

does hold uniformly in $S$. In particular there hold

$$
\partial_{\left(\xi_{j}\right)_{i}} W_{q}=-16 \pi \frac{\chi_{q} \delta_{q}^{2}}{\delta_{q}^{2}+\left|y_{\xi_{q}}(x)\right|^{2}} \partial_{\left(\xi_{j}\right)_{i}} G\left(\xi_{q}, \xi_{j}\right)+O\left(\delta^{2}|\log \delta|\right) \quad \forall q \neq j
$$


uniformly in $S$ and

$$
\partial_{\left(\xi_{j}\right)_{i}} W_{j}=8 \pi \partial_{\left(\xi_{j}\right)_{i}} G\left(x, \xi_{j}\right)+O\left(\delta^{2}|\log \delta|\right)
$$

locally uniformly in $S \backslash\left\{\xi_{j}\right\}$. Then we have that:

- for $q \neq l, j$

$$
\int_{S} \chi_{l} e^{-\varphi_{l}} e^{U_{l}} \partial_{\left(\xi_{j}\right)_{i}} W_{q} d v_{g}=O\left(\delta^{2}|\log \delta|\right)
$$

in view of $\chi_{l} \chi_{q} \equiv 0$;

- for $l \neq j$

$$
\begin{aligned}
\int_{S} \chi_{l} e^{-\varphi_{l}} e^{U_{l}} \partial_{\left(\xi_{j}\right)_{i}} W_{l} d v_{g} & =-16 \pi \partial_{\left(\xi_{j}\right)_{i}} G\left(\xi_{l}, \xi_{j}\right) \int_{S} \chi_{l}^{2} e^{-\varphi_{l}} e^{U_{l}} \frac{\delta_{l}^{2}}{\delta_{l}^{2}+\left|y_{\xi_{l}}(x)\right|^{2}} d v_{g}+O\left(\delta^{2}|\log \delta|\right) \\
& =-128 \pi \partial_{\left(\xi_{j}\right)_{i}} G\left(\xi_{l}, \xi_{j}\right) \int_{B_{\frac{r_{0}}{\delta_{l}}(0)}} \frac{d y}{\left(1+|y|^{2}\right)^{3}}+O\left(\delta^{2}|\log \delta|\right) \\
& =-64 \pi^{2} \partial_{\left(\xi_{j}\right)_{i}} G\left(\xi_{l}, \xi_{j}\right)+O\left(\delta^{2}|\log \delta|\right)
\end{aligned}
$$

and

$$
\int_{S} \chi_{l} e^{-\varphi_{l}} e^{U_{l}} \partial_{\left(\xi_{j}\right)_{i}} W_{j} d v_{g}=64 \pi^{2} \partial_{\left(\xi_{j}\right)_{i}} G\left(\xi_{l}, \xi_{j}\right)+O\left(\delta^{2}|\log \delta|\right)
$$

in view of Lemma 3.1. So we have that for $l \neq j$

$$
\int_{S} \chi_{l} e^{-\varphi_{l}} e^{U_{l}} \partial_{\left(\xi_{j}\right)_{i}} W d v_{g}=O\left(\delta^{2}|\log \delta|\right) .
$$

If $l=j$, by Lemma 3.1 we have that

$$
\begin{aligned}
& \int_{S} \chi_{j} e^{-\varphi_{j}} e^{U_{j}} \partial_{\left(\xi_{j}\right)_{i}} W d v_{g}=\int_{S} \chi_{j} e^{-\varphi_{j}} e^{U_{j}} \partial_{\left(\xi_{j}\right)_{i}} W_{j} d v_{g}+O\left(\delta^{2}|\log \delta|\right) \\
& =\int_{S} \chi_{j} e^{-\varphi_{j}} e^{U_{j}}\left[\chi_{j} \partial_{\left(\xi_{j}\right)_{i}}\left(U_{j}-\log \left(8 \delta_{j}^{2}\right)\right)+8 \pi \partial_{\left(\xi_{j}\right)_{i}} H\left(x, \xi_{j}\right)\right] d v_{g}+O\left(\delta^{2}|\log \delta|\right) \\
& =\partial_{\left(\xi_{j}\right)_{i}}\left[\int_{S} \chi_{j}^{2} e^{-\varphi_{j}} e^{U_{j}} d v_{g}\right]+\int_{S} \chi_{j}^{2} e^{-\varphi_{j}} e^{U_{j}} \partial_{\left(\xi_{j}\right)_{i}} \varphi_{j} d v_{g}-8 \pi \partial_{\left(\xi_{j}\right)_{i}} \log \rho_{j}\left(\xi_{j}\right)+\left.64 \pi^{2} \partial_{\left(\xi_{j}\right)_{i}} H\left(x, \xi_{j}\right)\right|_{x=\xi_{j}} \\
& +O\left(\delta^{2}|\log \delta|\right)=-8 \pi \partial_{\left(\xi_{j}\right)_{i}} \log \rho_{j}\left(\xi_{j}\right)+\left.64 \pi^{2} \partial_{\left(\xi_{j}\right)_{i}} H\left(x, \xi_{j}\right)\right|_{x=\xi_{j}}+O\left(\delta^{2}|\log \delta|\right)
\end{aligned}
$$

in view of $\partial_{\left(\xi_{j}\right)_{i}} \log \left(8 \delta_{j}^{2}\right)=\partial_{\left(\xi_{j}\right)_{i}} \log \rho_{j}\left(\xi_{j}\right), \partial_{\left(\xi_{j}\right)_{i}} \varphi_{j}\left(\xi_{j}\right)=0$ and

$$
\partial_{\left(\xi_{j}\right)_{i}}\left(\int_{S} \chi_{j}^{2} e^{-\varphi_{j}} e^{U_{j}} d v_{g}\right)=8 \partial_{\left(\xi_{j}\right)_{i}} \log \rho_{j}\left(\xi_{j}\right) \int_{\mathbb{R}^{2}} \frac{|y|^{2}-1}{\left(1+|y|^{2}\right)^{3}} d y+O\left(\delta^{2}\right)=O\left(\delta^{2}\right) .
$$

In view of $\int_{S} \partial_{\left(\xi_{j}\right)_{i}} W d v_{g}=0$ we can compute

$$
\begin{aligned}
-\int_{S} \Delta_{g} W \partial_{\left(\xi_{j}\right)_{i}} W d v_{g} & =\sum_{l=1}^{m} \int_{S} \chi_{l} e^{-\varphi_{l}} e^{U_{l}} \partial_{\left(\xi_{j}\right)_{i}} W d v_{g} \\
& =-8 \pi \partial_{\left(\xi_{j}\right)_{i}} \log \rho_{j}\left(\xi_{j}\right)+\left.64 \pi^{2} \partial_{\left(\xi_{j}\right)_{i}} H\left(x, \xi_{j}\right)\right|_{x=\xi_{j}}+O\left(\delta^{2}|\log \delta|\right) \\
& =-32 \pi^{2} \partial_{\left(\xi_{j}\right)_{i}} \varphi_{m}(\xi)+O\left(\delta^{2}|\log \delta|\right)
\end{aligned}
$$

in view of $G\left(x_{1}, x_{2}\right)=G\left(x_{2}, x_{1}\right)$ for all $x_{1} \neq x_{2}$ and $\left.\partial_{\left(\xi_{j}\right)_{i}} H\left(x, \xi_{j}\right)\right|_{x=\xi_{j}}=\frac{1}{2} \partial_{\left(\xi_{j}\right)_{i}}\left[H\left(\xi_{j}, \xi_{j}\right)\right]$. In order to give an expansion of the second term in $\partial_{\left(\xi_{j}\right)_{i}}\left[J_{\lambda}(W)\right]$, first observe that by Lemma 
2.1 there hold $k e^{W}=\frac{e^{\alpha \delta, \xi}}{8 \delta_{j}^{2}} \rho_{j} e^{U_{j}}\left[1+O\left(\delta^{2}\right)\right]$ uniformly in $B_{r_{0}}\left(\xi_{j}\right)$ and $k e^{W}=O(1)$ uniformly in $S \backslash \cup_{j=1}^{m} B_{r_{0}}\left(\xi_{j}\right)$. So we have that

$$
\begin{aligned}
& 8 e^{-\alpha_{\delta, \xi}} \int_{S} k e^{W} \partial_{\left(\xi_{j}\right)_{i}} W d v_{g}=\sum_{l, q=1}^{m} \delta_{q}^{-2} \int_{B_{r_{0}}\left(\xi_{q}\right)} \rho_{q} e^{U_{q}}\left(1+O\left(\delta^{2}\right)\right) \partial_{\left(\xi_{j}\right)_{i}} W_{l} d v_{g}+O(1) \\
& =\delta_{j}^{-2} \int_{B_{r_{0}}\left(\xi_{j}\right)} \rho_{j} e^{U_{j}}\left(1+O\left(\delta^{2}\right)\right) \partial_{\left(\xi_{j}\right)_{i}} W_{j} d v_{g}-16 \pi \sum_{l \neq j} \partial_{\left(\xi_{j}\right)_{i}} G\left(\xi_{l}, \xi_{j}\right) \int_{B_{r_{0}}\left(\xi_{l}\right)} \rho_{l} e^{U_{l}} \frac{d v_{g}}{\delta_{l}^{2}+\left|y_{\xi_{l}}(x)\right|^{2}} \\
& +8 \pi \sum_{l \neq q} \delta_{q}^{-2} \int_{B_{r_{0}}\left(\xi_{q}\right)} \rho_{q} e^{U_{q}} \partial_{\left(\xi_{j}\right)_{i}} H\left(x, \xi_{l}\right) d v_{g}+O(|\log \delta|)
\end{aligned}
$$

in view of $\partial_{\left(\xi_{j}\right)_{i}} W_{l}=8 \pi \partial_{\left(\xi_{j}\right)_{i}} H\left(x, \xi_{l}\right)+O\left(\delta^{2}|\log \delta|\right)$ in $B_{r_{0}}\left(\xi_{q}\right)$ when $q \neq l$. Since

$$
\partial_{\left(\xi_{j}\right)_{i}} W_{j}=\chi_{j} \partial_{\left(\xi_{j}\right)_{i}}\left[U_{j}-\log \left(8 \delta_{j}^{2}\right)\right]-4 \log \left|y_{\xi_{j}}(x)\right| \partial_{\left(\xi_{j}\right)_{i}} \chi_{j}+8 \pi \partial_{\left(\xi_{j}\right)_{i}} H\left(x, \xi_{j}\right)+O\left(\delta^{2}|\log \delta|\right),
$$

we have that

$$
\begin{aligned}
& \int_{B_{r_{0}}\left(\xi_{j}\right)} \rho_{j} e^{U_{j}} \partial_{\left(\xi_{j}\right)_{i}} W_{j} d v_{g} \\
& =\int_{S} \chi_{j} \rho_{j} e^{U_{j}} \partial_{\left(\xi_{j}\right)_{i}} U_{j} d v_{g}+\int_{S} \chi_{j} \rho_{j} e^{U_{j}}\left[8 \pi \partial_{\left(\xi_{j}\right)_{i}} H\left(x, \xi_{j}\right)-\partial_{\left(\xi_{j}\right)_{i}} \log \rho_{j}\left(\xi_{j}\right)\right]+O\left(\delta^{2}|\log \delta|\right) \\
& =\partial_{\left(\xi_{j}\right)_{i}}\left[\int_{S} \chi_{j} \rho_{j} e^{U_{j}} d v_{g}\right]-\partial_{\left(\xi_{j}\right)_{i}} \log \rho_{j}\left(\xi_{j}\right) \int_{S} \chi_{j} \rho_{j} e^{U_{j}}+O\left(\delta^{2}|\log \delta|\right)
\end{aligned}
$$

in view of $\partial_{\left(\xi_{j}\right)_{i}} \log \rho_{j}(x)=8 \pi \partial_{\left(\xi_{j}\right)_{i}} H\left(x, \xi_{j}\right)$. Since by the Taylor expansion of $e^{\hat{\varphi}_{j}}\left(\rho_{j} \circ y_{\xi_{j}}^{-1}\right)$ at 0 and the symmetries we have that

$$
\begin{aligned}
& \partial_{\left(\xi_{j}\right)_{i}}\left[\int_{S} \chi_{j} \rho_{j} e^{U_{j}} d v_{g}\right]=\int_{B_{2 r_{0}}(0)} \chi(|y|) \partial_{\left(\xi_{j}\right)_{i}}\left[e^{\hat{\varphi}_{j}(y)}\left(\rho_{j} \circ y_{\xi_{j}}^{-1}\right)(y)\right] \frac{8 \delta_{j}^{2}}{\left(\delta_{j}^{2}+|y|^{2}\right)^{2}} d y \\
& +\partial_{\left(\xi_{j}\right)_{i}} \log \rho_{j}\left(\xi_{j}\right) \int_{B_{2 r_{0}}(0)} \chi(|y|) e^{\hat{\varphi}_{j}(y)}\left(\rho_{j} \circ y_{\xi_{j}}^{-1}\right)(y) \frac{8 \delta_{j}^{2}\left(|y|^{2}-\delta_{j}^{2}\right)}{\left(\delta_{j}^{2}+|y|^{2}\right)^{3}} d y=8 \pi \partial_{\left(\xi_{j}\right)_{i} \rho_{j}\left(\xi_{j}\right)+O\left(\delta^{2}|\log \delta|\right)}
\end{aligned}
$$

in view of $\partial_{\left(\xi_{j}\right)_{i}}\left[e^{\hat{\varphi}_{j}(0)}\left(\rho_{j} \circ y_{\xi_{j}}^{-1}\right)(0)\right]=\partial_{\left(\xi_{j}\right)_{i}} \rho_{j}\left(\xi_{j}\right)$ and $\int_{\mathbb{R}^{2}} \frac{|y|^{2}-1}{\left(1+|y|^{2}\right)^{3}} d y=0$, by Lemma 3.1 we then deduce that

$$
\int_{B_{r_{0}}\left(\xi_{j}\right)} \rho_{j} e^{U_{j}} \partial_{\left(\xi_{j}\right)_{i}} W_{j} d v_{g}=O\left(\delta^{2}|\log \delta|\right) .
$$

Since by the Taylor expansion of $e^{\hat{\varphi}_{l}}\left(\rho_{l} \circ y_{\xi_{l}}^{-1}\right)$ at 0 and the symmetries we have that

$$
\begin{aligned}
\int_{B_{r_{0}}\left(\xi_{l}\right)} \rho_{l} e^{U_{l}} \frac{d v_{g}}{\delta_{l}^{2}+\left|y_{\xi_{l}}(x)\right|^{2}} & =\delta_{l}^{-2} \int_{B_{r_{0} / \delta_{l}}(0)}\left(\rho_{l} \circ y_{\xi_{l}}^{-1}\right)\left(\delta_{l} y\right) e^{\hat{\varphi}_{l}\left(\delta_{l} y\right)} \frac{8}{\left(1+|y|^{2}\right)^{3}} d y \\
& =\delta^{-2} \int_{\mathbb{R}^{2}} \frac{8}{\left(1+|y|^{2}\right)^{3}} d y+O(1)=\frac{4 \pi}{\delta^{2}}+O(1)
\end{aligned}
$$

by Lemma 3.1 we obtain that

$$
e^{-\alpha_{\delta, \xi}} \int_{S} k e^{W} \partial_{\left(\xi_{j}\right)_{i}} W d v_{g}=-\frac{8 \pi^{2}}{\delta^{2}} \sum_{l \neq j} \partial_{\left(\xi_{j}\right)_{i}} G\left(\xi_{l}, \xi_{j}\right)+\frac{8 \pi^{2}}{\delta^{2}} \sum_{l \neq j} \partial_{\left(\xi_{j}\right)_{i}} H\left(\xi_{l}, \xi_{j}\right)+O(|\log \delta|) .
$$

Since by (38) $\int_{S} k e^{W} d v_{g}=\frac{\pi m}{\delta^{2}} e^{\alpha_{\delta, \xi}}\left(1+O\left(\delta^{2}|\log \delta|\right)\right)$, we finally get that

$$
\begin{aligned}
& \int_{S} \frac{k e^{W}}{\int_{S} k e^{W} d v_{g}} \partial_{\left(\xi_{j}\right)_{i}} W d v_{g} \\
& =-\frac{8 \pi}{m} \sum_{l \neq j} \partial_{\left(\xi_{j}\right)_{i}} G\left(\xi_{l}, \xi_{j}\right)+\frac{8 \pi}{m} \sum_{l \neq j} \partial_{\left(\xi_{j}\right)_{i}} H\left(\xi_{l}, \xi_{j}\right)+O\left(\delta^{2}|\log \delta|\right)=O\left(\delta^{2}|\log \delta|\right)
\end{aligned}
$$


in view of $G\left(\xi_{l}, \xi_{j}\right)=H\left(\xi_{l}, \xi_{j}\right)$ for $l \neq j$. In conclusion, by (41)-(42) we can write

$$
\partial_{\left(\xi_{j}\right)_{i}}\left[J_{8 \pi m}(W)\right]=-32 \pi^{2} \partial_{\left(\xi_{j}\right)_{i}} \varphi_{m}(\xi)+O\left(\delta^{2}|\log \delta|\right) .
$$

By (25) we have that $\partial_{\left(\xi_{j}\right)_{i}}\left[J_{\lambda}(W)\right]=\partial_{\left(\xi_{j}\right)_{i}}\left[J_{8 \pi}(W)\right]+O\left(\delta^{2}|\log \delta|\right)$, and the proof is complete.

Finally, we address the expansions for the derivatives of $J_{\lambda}(W)$ in $\delta$.

Proof (of (35) in $C^{2}(\mathbb{R})$ ): We just focus on the first and second derivative of $J_{\lambda}(W)$ in $\delta$. Since $\partial_{\delta}=\rho_{l}^{\frac{1}{2}}\left(\xi_{l}\right) \partial_{\delta_{l}}$ in view of (24), arguing as in Lemma 2.1] it is easy to show that

$$
\begin{aligned}
& \rho_{l}^{-\frac{1}{2}}\left(\xi_{l}\right) \partial_{\delta} W_{l}=-\chi_{l} \frac{4 \delta_{l}}{\delta_{l}^{2}+\left|y_{\xi_{l}}(x)\right|^{2}}+\beta_{\delta_{l}, \xi_{l}}-4 \delta_{l} F_{\xi_{l}}+O\left(\delta^{3}|\log \delta|\right) \\
& \rho_{l}^{-1}\left(\xi_{l}\right) \partial_{\delta \delta} W_{l}=4 \chi_{l} \frac{\delta_{l}^{2}-\left|y_{\xi_{l}}(x)\right|^{2}}{\left(\delta_{l}^{2}+\left|y_{\xi_{l}}(x)\right|^{2}\right)^{2}}+\gamma_{\delta_{l}, \xi_{l}}-4 F_{\xi_{l}}+O\left(\delta^{2}|\log \delta|\right)
\end{aligned}
$$

do hold uniformly in $S$, where

$$
\beta_{\delta_{l}, \xi_{l}}=-\frac{8 \pi}{|S|} \delta_{l} \log \delta_{l}+\frac{4 \delta_{l}}{|S|}\left(\int_{\mathbb{R}^{2}} \chi(|y|) \frac{e^{\hat{\varphi}_{\xi}(y)}-1}{|y|^{2}} d y-\int_{\mathbb{R}^{2}} \frac{\chi^{\prime}(|y|) \log |y|}{|y|} d y\right)
$$

and

$$
\gamma_{\delta_{l}, \xi_{l}}=-\frac{8 \pi}{|S|} \log \delta_{l}+\frac{4}{|S|}\left(\int_{\mathbb{R}^{2}} \chi(|y|) \frac{e^{\hat{\varphi}_{\xi}(y)}-1}{|y|^{2}} d y-2 \pi-\int_{\mathbb{R}^{2}} \frac{\chi^{\prime}(|y|) \log |y|}{|y|} d y\right) .
$$

By Lemma 3.1 we then have that

$$
\begin{aligned}
& \rho_{l}^{-\frac{1}{2}}\left(\xi_{l}\right) \int_{S} \chi_{j} e^{-\varphi_{j}} e^{U_{j}} \partial_{\delta} W_{l} d v_{g} \\
&=-\int_{S} \chi_{j} \chi_{l} e^{-\varphi_{j}} e^{U_{j}} \frac{4 \delta_{l}}{\delta_{l}^{2}+\left|y_{\xi_{l}}(x)\right|^{2}} d v_{g}+8 \pi \beta_{\delta_{l}, \xi_{l}}-32 \pi \delta_{l} F_{\xi_{l}}\left(\xi_{j}\right)+O\left(\delta^{3}|\log \delta|^{2}\right) \\
&=-\frac{32}{\delta_{j}} \delta_{j l} \int_{B_{r_{0} / \delta_{j}}(0)} \frac{d y}{\left(1+|y|^{2}\right)^{3}}+8 \pi \beta_{\delta_{l}, \xi_{l}}-32 \pi \delta_{l} F_{\xi_{l}}\left(\xi_{j}\right)+O\left(\delta^{3}|\log \delta|^{2}\right) \\
&=-\frac{16 \pi}{\delta_{j}} \delta_{j l}+8 \pi \beta_{\delta_{l}, \xi_{l}}-32 \pi \delta_{l} F_{\xi_{l}}\left(\xi_{j}\right)+O\left(\delta^{3}|\log \delta|^{2}\right), \\
& \rho_{l}^{-1}\left(\xi_{l}\right) \int_{S} \chi_{j} e^{-\varphi_{j}} e^{U_{j}} \partial_{\delta \delta} W_{l} d v_{g} \\
&=4 \int_{S} \chi_{j} \chi_{l} e^{-\varphi_{j}} e^{U_{j}} \frac{\delta_{l}^{2}-\left|y_{\xi_{l}}(x)\right|^{2}}{\left(\delta_{l}^{2}+\left|y_{\xi_{l}}(x)\right|^{2}\right)^{2}} d v_{g}+8 \pi \gamma_{\delta_{l}, \xi_{l}}-32 \pi F_{\xi_{l}}\left(\xi_{j}\right)+O\left(\delta^{2}|\log \delta|^{2}\right) \\
&=\frac{32}{\delta_{j}^{2}} \delta_{j l} \int_{B_{r_{0} / \delta_{j}}(0)}\left[\frac{2}{\left(1+|y|^{2}\right)^{4}}-\frac{1}{\left(1+|y|^{2}\right)^{3}}\right] d y+8 \pi \gamma_{\delta_{l}, \xi_{l}}-32 \pi F_{\xi_{l}}\left(\xi_{j}\right)+O\left(\delta^{2}|\log \delta|^{2}\right) \\
&=\frac{16 \pi}{3 \delta_{j}^{2}} \delta_{j l}+8 \pi \gamma_{\delta_{l}, \xi_{l}}-32 \pi F_{\xi_{l}}\left(\xi_{j}\right)+O\left(\delta^{2}|\log \delta|^{2}\right)
\end{aligned}
$$

and

$$
\begin{aligned}
& \rho_{l}^{-\frac{1}{2}}\left(\xi_{l}\right) \int_{S} \chi_{j} e^{-\varphi_{j}} e^{U_{j}} \partial_{\delta} U_{j} \partial_{\delta} W_{l} d v_{g}=\frac{2}{\delta} \rho_{l}^{-\frac{1}{2}}\left(\xi_{l}\right) \int_{S} \chi_{j} e^{-\varphi_{j}} e^{U_{j}} \frac{\left|y_{\xi_{j}}(x)\right|^{2}-\delta_{j}^{2}}{\delta_{j}^{2}+\left|y_{\xi_{j}}(x)\right|^{2}} \partial_{\delta} W_{l} d v_{g} \\
& =8 \rho_{j}\left(\xi_{j}\right)^{\frac{1}{2}} \delta_{j l} \int_{S} \chi_{j} e^{-\varphi_{j}} e^{U_{j}} \frac{\delta_{j}^{2}-\left|y_{\xi_{j}}(x)\right|^{2}}{\left(\delta_{j}^{2}+\left|y_{\xi_{j}}(x)\right|^{2}\right)^{2}} d v_{g}+\frac{16}{\delta}\left(\beta_{\delta_{l}, \xi_{l}}-4 \delta_{l} F_{\xi_{l}}\left(\xi_{j}\right)\right) \int_{\mathbb{R}^{2}} \frac{|y|^{2}-1}{\left(1+|y|^{2}\right)^{3}} d y \\
& +O\left(\delta^{2}|\log \delta|\right)=\frac{32 \pi}{3 \delta_{j}^{2}} \rho_{j}\left(\xi_{j}\right)^{\frac{1}{2}} \delta_{j l}+O\left(\delta^{\gamma}\right)
\end{aligned}
$$


in view of $\int_{\mathbb{R}^{2}} \frac{|y|^{2}-1}{\left(1+|y|^{2}\right)^{3}} d y=0$, where $\delta_{j l}$ denotes the Kronecker's symbol. Since $\int_{S} \partial_{\delta} W d v_{g}=$ $\int_{S} \partial_{\delta \delta} W d v_{g}=0$, we then deduce the following expansions:

$$
\begin{aligned}
\int_{S}\left(-\Delta_{g} W\right) \partial_{\delta} W d v_{g} & =\sum_{j, l=1}^{m} \int_{S} \chi_{j} e^{-\varphi_{j}} e^{U_{j}} \partial_{\delta} W_{l} d v_{g} \\
& =-\frac{16 \pi m}{\delta}+8 \pi m \sum_{l=1}^{m} \rho_{l}^{\frac{1}{2}}\left(\xi_{l}\right) \beta_{\delta_{l}, \xi_{l}}-32 \pi \delta \sum_{j, l=1}^{m} \rho_{l}\left(\xi_{l}\right) F_{\xi_{l}}\left(\xi_{j}\right)+O\left(\delta^{3}|\log \delta|^{2}\right) \\
\int_{S}\left(-\Delta_{g} W\right) \partial_{\delta \delta} W d v_{g} & =\sum_{j, l=1}^{m} \int_{S} \chi_{j} e^{-\varphi_{j}} e^{U_{j}} \partial_{\delta \delta} W_{l} d v_{g} \\
& =\frac{16 \pi m}{3 \delta^{2}}+8 \pi m \sum_{l=1}^{m} \rho_{l}\left(\xi_{l}\right) \gamma_{\delta_{l}, \xi_{l}}-32 \pi \sum_{j, l=1}^{m} \rho_{l}\left(\xi_{l}\right) F_{\xi_{l}}\left(\xi_{j}\right)+O\left(\delta^{2}|\log \delta|^{2}\right)
\end{aligned}
$$

and

$$
\int_{S}-\Delta_{g}\left(\partial_{\delta} W\right) \partial_{\delta} W d v_{g}=\sum_{j, l=1}^{m} \int_{S} \chi_{j} e^{-\varphi_{j}} e^{U_{j}} \partial_{\delta} U_{j} \partial_{\delta} W_{l} d v_{g}=\frac{32 \pi m}{3 \delta^{2}}+O\left(\delta^{\gamma}\right)
$$

as $\delta \rightarrow 0$. Since by Lemma 2.1 there hold

$$
k e^{W}=\frac{e^{\alpha_{\delta, \xi}-2 F_{\delta, \xi}(x)}}{8 \delta_{j}^{2}} \rho_{j} e^{U_{j}}\left[1+O\left(\delta^{4}|\log \delta|\right)\right]
$$

uniformly in $B_{r_{0}}\left(\xi_{j}\right)$ and $k e^{W}=O(1), \partial_{\delta} W=O(\delta|\log \delta|)$ uniformly in $S \backslash \cup_{j=1}^{m} B_{r_{0}}\left(\xi_{j}\right)$, by Lemma 3.1 we can write that

$$
\begin{aligned}
& \int_{S} k e^{W} \partial_{\delta} W d v_{g}=\sum_{j, l=1}^{m} \int_{B_{r_{0}}\left(\xi_{j}\right)} k e^{W} \partial_{\delta} W_{l} d v_{g}+O(\delta|\log \delta|) \\
& =-\sum_{j=1}^{m} \frac{e^{\alpha_{\delta, \xi}}}{2 \delta} \int_{B_{r_{0}}\left(\xi_{j}\right)} e^{-2 F_{\delta, \xi}(x)} \frac{\rho_{j} e^{U_{j}}}{\delta_{j}^{2}+\left|y_{\xi_{j}}(x)\right|^{2}} d v_{g}+\pi \frac{e^{\alpha_{\delta, \xi}}}{\delta^{2}}\left(m \sum_{l=1}^{m} \rho_{l}^{\frac{1}{2}}\left(\xi_{l}\right) \beta_{\delta_{l}, \xi_{l}}-4 \sum_{j, l=1}^{m} \rho_{l}^{\frac{1}{2}}\left(\xi_{l}\right) \delta_{l} F_{\xi_{l}}\left(\xi_{j}\right)\right) \\
& +O(|\log \delta|)=-\sum_{j=1}^{m} \frac{e^{\alpha_{\delta, \xi}}}{2 \delta}\left(\frac{4 \pi}{\delta^{2}} e^{-2 F_{\delta, \xi}\left(\xi_{j}\right)}+\pi\left(\Delta_{g} \rho_{j}\left(\xi_{j}\right)-2 K\left(\xi_{j}\right) \rho_{j}\left(\xi_{j}\right)\right)\right) \\
& +\pi \frac{e^{\alpha_{\delta, \xi}}}{\delta^{2}}\left(m \sum_{l=1}^{m} \rho_{l}^{\frac{1}{2}}\left(\xi_{l}\right) \beta_{\delta_{l}, \xi_{l}}-4 \sum_{j, l=1}^{m} \rho_{l}^{\frac{1}{2}}\left(\xi_{l}\right) \delta_{l} F_{\xi_{l}}\left(\xi_{j}\right)\right)+O\left(\delta^{-1+\gamma}\right) \\
& =\pi \frac{e^{\alpha_{\delta, \xi}}}{\delta^{2}}\left(-\frac{2 m}{\delta}+m \sum_{l=1}^{m} \rho_{l}^{\frac{1}{2}}\left(\xi_{l}\right) \beta_{\delta_{l}, \xi_{l}}-\frac{\delta}{8 \pi} A(\xi)+O\left(\delta^{1+\gamma}\right)\right)
\end{aligned}
$$

in view of (37) and

$$
\frac{1}{\delta} \sum_{j=1}^{m} e^{-2 F_{\delta, \xi}\left(\xi_{j}\right)}=\frac{m}{\delta}-\frac{2}{\delta} \sum_{j, l=1}^{m} \delta_{l}^{2} F_{\xi_{l}}\left(\xi_{j}\right)+O\left(\delta^{3}\right)=\frac{m}{\delta}-2 \sum_{j, l=1}^{m} \rho_{l}^{\frac{1}{2}}\left(\xi_{l}\right) \delta_{l} F_{\xi_{l}}\left(\xi_{j}\right)+O\left(\delta^{3}\right) .
$$

Combining with (38) we then get that

$$
\begin{aligned}
\frac{\int_{S} k e^{W} \partial_{\delta} W d v_{g}}{\int_{S} k e^{W} d v_{g}}= & -\frac{2}{\delta}+\sum_{l=1}^{m} \rho_{l}^{\frac{1}{2}}\left(\xi_{l}\right) \beta_{\delta_{l}, \xi_{l}}-\frac{\delta}{8 \pi m} A(\xi)-\frac{A(\xi)}{4 \pi m} \delta \log \delta+\frac{B(\xi)}{4 \pi m} \delta \\
& -\frac{4}{m \delta} \sum_{j=1}^{m} F_{\delta, \xi}\left(\xi_{j}\right)+o(\delta),
\end{aligned}
$$


which yields to

$$
\begin{aligned}
\partial_{\delta}\left[J_{8 \pi m}(W)\right] & =\int_{S}\left(-\Delta_{g} W\right) \partial_{\delta} W d v_{g}-8 \pi m \frac{\int_{S} k e^{W} \partial_{\delta} W d v_{g}}{\int_{S} k e^{W} d v_{g}} \\
& =2 A(\xi) \delta \log \delta+[A(\xi)-2 B(\xi)] \delta+o(\delta) .
\end{aligned}
$$

Since by (25) and (50) there holds

$$
-(\lambda-8 \pi m) \frac{\int_{S} k e^{W} \partial_{\delta} W d v_{g}}{\int_{S} k e^{W} d v_{g}}=\frac{2(\lambda-8 \pi m)}{\delta}+O\left(\delta^{3}|\log \delta|^{2}\right),
$$

by (51) we deduce the validity of (35) for the first derivative in $\delta$.

Towards the expansion of the second derivative, we proceed in a similar way with the aid of the expansion for $\partial_{\delta \delta} W_{l}$. Since

$$
k e^{W}=\frac{e^{\alpha_{\delta, \xi}-2 F_{\delta, \xi}(x)}}{8 \delta_{j}^{2}} \rho_{j} e^{U_{j}}\left[1+O\left(\delta^{4}|\log \delta|\right)\right]
$$

and $k e^{W}=O(1), \partial_{\delta \delta} W+\left(\partial_{\delta} W\right)^{2}=O(|\log \delta|)$ do hold uniformly in $B_{r_{0}}\left(\xi_{j}\right)$ and $S \backslash \cup_{j=1}^{m} B_{r_{0}}\left(\xi_{j}\right)$, respectively, by Lemma 3.1 we can write that

$$
\begin{aligned}
& \int_{S} k e^{W}\left[\partial_{\delta \delta} W+\left(\partial_{\delta} W\right)^{2}\right] d v_{g}=\sum_{j=1}^{m} \int_{B_{r_{0}}\left(\xi_{j}\right)} k e^{W}\left[\partial_{\delta \delta} W+\left(\partial_{\delta} W\right)^{2}\right] d v_{g}+O(|\log \delta|) \\
& =\sum_{j=1}^{m} \frac{e^{\alpha_{\delta, \xi}}}{2 \delta^{2}} \int_{B_{r_{0}}\left(\xi_{j}\right)} e^{-2 F_{\delta, \xi}(x)} \rho_{j} e^{U_{j}} \frac{5 \delta_{j}^{2}-\left|y_{\xi_{j}}(x)\right|^{2}}{\left(\delta_{j}^{2}+\left|y_{\xi_{j}}(x)\right|^{2}\right)^{2}} d v_{g} \\
& -\sum_{j=1}^{m} \frac{e^{\alpha_{\delta, \xi}}}{\delta} \int_{B_{r_{0}}\left(\xi_{j}\right)} e^{-2 F_{\delta, \xi}(x)} \frac{\rho_{j} e^{U_{j}}}{\delta_{j}^{2}+\left|y_{\xi_{j}}(x)\right|^{2}}\left(\sum_{l=1}^{m} \rho_{l}^{\frac{1}{2}}\left(\xi_{l}\right) \beta_{\delta_{l}, \xi_{l}}-4 \delta \sum_{l=1}^{m} \rho_{l}\left(\xi_{l}\right) F_{\xi_{l}}\right) d v_{g} \\
& +\pi \frac{e^{\alpha_{\delta, \xi}}}{\delta^{2}}\left(m \sum_{l=1}^{m} \rho_{l}\left(\xi_{l}\right) \gamma_{\delta_{l}, \xi_{l}}-4 \sum_{j, l=1}^{m} \rho_{l}\left(\xi_{l}\right) F_{\xi_{l}}\left(\xi_{j}\right)\right)+O\left(|\log \delta|^{2}\right),
\end{aligned}
$$

and then

$$
\begin{aligned}
& \frac{\delta^{2} e^{-\alpha_{\delta, \xi}}}{\pi} \int_{S} k e^{W}\left[\partial_{\delta \delta} W+\left(\partial_{\delta} W\right)^{2}\right] d v_{g}=\sum_{j=1}^{m}\left(\frac{6}{\delta^{2}} e^{-2 F_{\delta, \xi}\left(\xi_{j}\right)}+\frac{1}{2}\left(\Delta_{g} \rho_{j}\left(\xi_{j}\right)-2 K\left(\xi_{j}\right) \rho_{j}\left(\xi_{j}\right)\right)\right) \\
& -\frac{4}{\delta} \sum_{j=1}^{m}\left(\sum_{l=1}^{m} \rho_{l}^{\frac{1}{2}}\left(\xi_{l}\right) \beta_{\delta_{l}, \xi_{l}}-4 \delta \sum_{l=1}^{m} \rho_{l}\left(\xi_{l}\right) F_{\xi_{l}}\left(\xi_{j}\right)\right)+m \sum_{l=1}^{m} \rho_{l}\left(\xi_{l}\right) \gamma_{\delta_{l}, \xi_{l}}-4 \sum_{j, l=1}^{m} \rho_{l}\left(\xi_{l}\right) F_{\xi_{l}}\left(\xi_{j}\right)+O\left(\delta^{\gamma}\right) \\
& =\frac{6 m}{\delta^{2}}+m \sum_{l=1}^{m} \rho_{l}\left(\xi_{l}\right) \gamma_{\delta_{l}, \xi_{l}}-\frac{4 m}{\delta} \sum_{l=1}^{m} \rho_{l}^{\frac{1}{2}}\left(\xi_{l}\right) \beta_{\delta_{l}, \xi_{l}}+\frac{A(\xi)}{8 \pi}+O\left(\delta^{\gamma}\right)
\end{aligned}
$$

in view of (37) and (49). Combining with (38) we then get that

$$
\begin{aligned}
& \frac{\int_{S} k e^{W}\left[\partial_{\delta \delta} W+\left(\partial_{\delta} W\right)^{2}\right] d v_{g}}{\int_{S} k e^{W} d v_{g}}=\frac{6}{\delta^{2}}+\frac{3 A(\xi)}{4 \pi m} \log \delta+\sum_{l=1}^{m} \rho_{l}\left(\xi_{l}\right) \gamma_{\delta_{l}, \xi_{l}} \\
& -\frac{4}{\delta} \sum_{l=1}^{m} \rho_{l}^{\frac{1}{2}}\left(\xi_{l}\right) \beta_{\delta_{l}, \xi_{l}}+\frac{A(\xi)}{8 \pi m}-\frac{3 B(\xi)}{4 \pi m}+\frac{12}{m \delta^{2}} \sum_{j=1}^{m} F_{\delta, \xi}\left(\xi_{j}\right)+o(1) .
\end{aligned}
$$


Since

$$
\begin{aligned}
\partial_{\delta \delta}\left[J_{\lambda}(W)\right]= & \int_{S}\left(-\Delta_{g} W\right) \partial_{\delta \delta} W d v_{g}-\lambda \frac{\int_{S} k e^{W}\left[\partial_{\delta \delta} W+\left(\partial_{\delta} W\right)^{2}\right] d v_{g}}{\int_{S} k e^{W} d v_{g}} \\
& +\int_{S}\left(-\Delta_{g} \partial_{\delta} W\right) \partial_{\delta} W d v_{g}+\lambda\left(\frac{\int_{S} k e^{W} \partial_{\delta} W d v_{g}}{\int_{S} k e^{W} d v_{g}}\right)^{2},
\end{aligned}
$$

by (47), (48), (50) and (52) we deduce that

$$
\partial_{\delta \delta}\left[J_{8 \pi m}(W)\right]=2 A(\xi) \log \delta+3 A(\xi)-2 B(\xi)+o(1) .
$$

Since by (25), (50) and (52)

$$
(\lambda-8 \pi m)\left[\frac{\int_{S} k e^{W}\left[\partial_{\delta \delta} W+\left(\partial_{\delta} W\right)^{2}\right] d v_{g}}{\int_{S} k e^{W} d v_{g}}-\left(\frac{\int_{S} k e^{W} \partial_{\delta} W d v_{g}}{\int_{S} k e^{W} d v_{g}}\right)^{2}\right]=2 \frac{\lambda-8 \pi m}{\delta^{2}}+O\left(\delta^{2}|\log \delta|^{2}\right),
$$

by (53) we deduce the validity of (35) also for the second derivative in $\delta$, and the proof is complete.

\section{Variational reduction and proof of main results}

In the so-called nonlinear Lyapunov-Schimdt reduction, the first step is the solvability theory for the operator $L$ given in (27), obtained as the linearization of (1) at the approximating solution $W$. As $\delta \rightarrow 0$ observe that formally the operator $L$, scaled and centered at 0 by setting $y=y_{\xi_{j}}(x) / \delta_{j}$, approaches $\hat{L}$ defined in $\mathbb{R}^{2}$ as

$$
\hat{L}(\phi)=\Delta \phi+\frac{8}{\left(1+|y|^{2}\right)^{2}}\left(\phi-\frac{1}{\pi} \int_{\mathbb{R}^{2}} \frac{\phi(z)}{\left(1+|z|^{2}\right)^{2}} d z\right) .
$$

Due to the intrinsic invariances, the kernel of $\hat{L}$ in $L^{\infty}\left(\mathbb{R}^{2}\right)$ is non-empty and is spanned by 1 and $Y_{j}, j=0,1,2$, where

$$
Y_{i}(y)=\frac{4 y_{i}}{1+|y|^{2}}, \quad i=1,2, \quad \text { and } \quad Y_{0}(y)=2 \frac{1-|y|^{2}}{1+|y|^{2}} .
$$

Since 25, 33 it is by now rather standard to show the invertibility of $L$ in a suitable "orthogonal" space, and a sketched proof of it will be given in Appendix A. However, for Dirichlet Liouvilletype equations on bounded domains as in 25, 33, the corresponding limiting operator $\tilde{L}$ takes the form $\tilde{L}(\phi)=\Delta \phi+\frac{8}{\left(1+|y|^{2}\right)^{2}} \phi$ and the function 1 does not belong to its kernel, making possible to disregard the "dilation parameters" $\delta_{i}$ in the reduction. As we will see, one additional parameter $\delta$ is needed in the reduction and in this respect our problem displays a new feature w.r.t. Dirichlet Liouville-type equations, making our situation very similar to the one arising in the study of critical problems in higher dimension.

To be more precise, for $i=0,1,2$ and $j=1, \ldots, m$ introduce the functions

$$
Z_{i j}(x)=Y_{i}\left(\frac{y_{\xi_{j}}(x)}{\delta_{j}}\right)= \begin{cases}2 \frac{\delta_{j}^{2}-\left|y_{\xi_{j}}(x)\right|^{2}}{\delta_{j}^{2}+\left|y_{\xi_{j}}(x)\right|^{2}} & \text { for } i=0 \\ \frac{4 \delta_{j}\left(y_{\xi_{j}}(x)\right)_{i}}{\delta_{j}^{2}+\left|y_{\xi_{j}}(x)\right|^{2}} & \text { for } i=1,2\end{cases}
$$

and set $Z=\sum_{l=1}^{m} Z_{0 l}$. For $i=1,2$ and $j=1, \ldots, m$, let $P Z, P Z_{i j}$ be the projections of $Z, Z_{i j}$ as the solutions in $\bar{H}$ of

$$
\begin{aligned}
\Delta_{g} P Z & =\chi_{j} \Delta_{g} Z-\frac{1}{|S|} \int_{S} \chi_{j} \Delta_{g} Z d v_{g} \\
\Delta_{g} P Z_{i j} & =\chi_{j} \Delta_{g} Z_{i j}-\frac{1}{|S|} \int_{S} \chi_{j} \Delta_{g} Z_{i j} d v_{g} .
\end{aligned}
$$

In Appendix A we will prove the following result: 
Proposition 4.1. There exists $\delta_{0}>0$ so that for all $0<\delta \leq \delta_{0}, h \in C(S)$ with $\int_{S} h d v_{g}=0$, $\xi \in \Xi$ there is a unique solution $\phi \in \bar{H} \cap W^{2,2}(S)$ and $c_{0}, c_{i j} \in \mathbb{R}$ of

$$
\begin{cases}L(\phi)=h+c_{0} \Delta_{g} P Z+\sum_{i=1}^{2} \sum_{j=1}^{m} c_{i j} \Delta_{g} P Z_{i j} & \text { in } S \\ \int_{S} \phi \Delta_{g} P Z d v_{g}=\int_{S} \phi \Delta_{g} P Z_{i j} d v_{g}=0 & \forall i=1,2, j=1, \ldots, m .\end{cases}
$$

Moreover, the map $(\delta, \xi) \mapsto\left(\phi, c_{0}, c_{i j}\right)$ is twice-differentiable in $\delta$ and one-differentiable in $\xi$ with

$$
\begin{aligned}
& \|\phi\|_{\infty} \leq C|\log \delta|\|h\|_{*}, \quad\left|c_{0}\right|+\sum_{i=1}^{2} \sum_{j=1}^{m}\left|c_{i j}\right| \leq C\|h\|_{*} \\
& \left\|\partial_{\delta} \phi\right\|_{\infty}+\sum_{i=1}^{2} \sum_{j=1}^{m}\left\|\partial_{\left(\xi_{j}\right)_{i}} \phi\right\|_{\infty}+\frac{\delta}{|\log \delta|}\left\|\partial_{\delta \delta} \varphi\right\|_{\infty} \leq C \frac{|\log \delta|^{2}}{\delta}\|h\|_{*}
\end{aligned}
$$

for some $C>0$.

Let us recall that $u=W+\phi$ solves (11) if $\phi \in \bar{H}$ does satisfy (26). Since the operator $L$ is not fully invertible, in view of Proposition 4.1 one can solve the nonlinear problem (26) just up to a linear combination of $\Delta_{g} P Z$ and $\Delta_{g} P Z_{i j}$, as explained in the following (see Appendix B for the proof):

Proposition 4.2. There exists $\delta_{0}>0$ so that for all $0<\delta \leq \delta_{0}, \xi \in \Xi$ problem

$$
\begin{cases}L(\phi)=-[R+N(\phi)]+c_{0} \Delta_{g} P Z+\sum_{i=1}^{2} \sum_{j=1}^{m} c_{i j} \Delta_{g} P Z_{i j} & \text { in } S \\ \int_{S} \phi \Delta_{g} P Z d v_{g}=\int_{S} \phi \Delta_{g} P Z_{i j} d v_{g}=0 & \forall i=1,2, j=1, \ldots, m\end{cases}
$$

admits a unique solution $\phi(\delta, \xi) \in \bar{H} \cap W^{2,2}(S)$ and $c_{0}(\delta, \xi), c_{i j}(\delta, \xi) \in \mathbb{R}, i=1,2$ and $j=$ $1, \ldots, m$, where $\delta_{j}>0$ are as in (24) and $N, R$ are given by (28), (29), respectively. Moreover, the map $(\delta, \xi) \mapsto\left(\phi(\delta, \xi), c_{0}(\delta, \xi), c_{i j}(\delta, \xi)\right)$ is twice-differentiable in $\delta$ and one-differentiable in $\xi$ with

$$
\begin{aligned}
& \|\phi\|_{\infty} \leq C\left(\delta|\log \delta|\left|\nabla \varphi_{m}(\xi)\right|_{g}+\delta^{2-\sigma}|\log \delta|^{2}\right) \\
& \left\|\partial_{\delta} \phi\right\|_{\infty}+\sum_{i=1}^{2} \sum_{j=1}^{m}\left\|\partial_{\left(\xi_{j}\right)_{i}} \phi\right\|_{\infty} \leq C\left(|\log \delta|^{2}\left|\nabla \varphi_{m}(\xi)\right|_{g}+\delta^{1-\sigma}|\log \delta|^{3}\right) \\
& \left\|\partial_{\delta \delta} \phi\right\|_{\infty} \leq C\left(\delta^{-1}|\log \delta|^{3}\left|\nabla \varphi_{m}(\xi)\right|_{g}+\delta^{-\sigma}|\log \delta|^{4}\right) .
\end{aligned}
$$

The function $W+\phi(\delta, \xi)$ will be a true solution of (26) if $\delta$ and $\xi$ are such that $c_{0}(\delta, \xi)=$ $c_{i j}(\delta, \xi)=0$ for all $i=1,2$, and $j=1, \ldots, m$. This problem is equivalent to finding critical points of the reduced energy $E_{\lambda}(\delta, \xi)=J_{\lambda}(W+\phi(\delta, \xi))$, where $J_{\lambda}$ is given by (4), as stated in

Lemma 4.3. There exists $\delta_{0}$ such that, if $(\delta, \xi) \in\left(0, \delta_{0}\right] \times \Xi$ is a critical point of $E_{\lambda}$, then $u=W+\phi(\delta, \xi)$ is a solution of (11), where $\delta_{i}$ are given by (24).

Once equation (11) has been reduced to the search of c.p.'s for $E_{\lambda}$, it becomes crucial to show that the main asymptotic term of $E_{\lambda}$ is given by $J_{\lambda}(W)$, for which an expansion has been given in Theorem 3.2. More precisely, by the estimates in Appendix B we have that

Theorem 4.4. Assume (24)-(25). The following expansion does hold

$$
\begin{aligned}
E_{\lambda}(\delta, \xi)= & -8 \pi m-\lambda \log (\pi m)-32 \pi^{2} \varphi_{m}(\xi)+2(\lambda-8 \pi m) \log \delta+A(\xi) \delta^{2} \log \delta \\
& -B(\xi) \delta^{2}+o\left(\delta^{2}\right)+r_{\lambda}(\delta, \xi)
\end{aligned}
$$


in $C^{2}(\mathbb{R})$ and $C^{1}(\Xi)$ as $\delta \rightarrow 0^{+}$, where $\varphi_{m}(\xi), A(\xi)$ and $B(\xi)$ are given by (5), (7) and (12), respectively. The term $r_{\lambda}(\delta, \xi)$ satisfies

$$
\left|r_{\lambda}(\delta, \xi)\right|+\frac{\delta}{|\log \delta|}\left|\nabla r_{\lambda}(\delta, \xi)\right|+\frac{\delta^{2}}{|\log \delta|^{2}}\left|\partial_{\delta \delta} r_{\lambda}(\delta, \xi)\right| \leq C \delta^{2}|\log \delta|\left|\nabla \varphi_{m}(\xi)\right|_{g}^{2}
$$

for some $C>0$ independent of $(\delta, \xi) \in\left(0, \delta_{0}\right] \times \Xi$.

We are now in position to establish the main result stated in the Introduction.

Proof (of Theorem 1.5): According to Lemma 4.3, we just need to find a critical point of $E_{\lambda}(\delta, \xi)$. By Theorem 4.4 for $\lambda>8 \pi m$ we have that

$$
\begin{aligned}
\frac{\left(\delta \partial_{\delta} E_{\lambda}\right)(\sqrt{\lambda-8 \pi m} \mu, \xi)}{\lambda-8 \pi m}= & 2+A(\xi) \log (\lambda-8 \pi m) \mu^{2}+2 A(\xi) \mu^{2} \log \mu+(A(\xi)-2 B(\xi)) \mu^{2} \\
& +o(1)+O\left(\mu^{2}|\log (\sqrt{\lambda-8 \pi m} \mu)|^{2}\left|\nabla \varphi_{m}(\xi)\right|_{g}^{2}\right)
\end{aligned}
$$

and

$$
\begin{aligned}
\frac{\left(\delta^{2} \partial_{\delta \delta} E_{\lambda}\right)(\sqrt{\lambda-8 \pi m} \mu, \xi)}{\lambda-8 \pi m}= & -2+A(\xi) \mu^{2}[2 \log \mu+\log (\lambda-8 \pi m)+3]-2 B(\xi) \mu^{2} \\
& +o(1)+O\left(\mu^{2}|\log (\sqrt{\lambda-8 \pi m} \mu)|^{3}\left|\nabla \varphi_{m}(\xi)\right|_{g}^{2}\right)
\end{aligned}
$$

as $\lambda \rightarrow 8 \pi m$. By assumption we can find $a_{0}>0$ small so that $B(\xi)>0$ for all $\xi \in U$ with $|A(\xi)| \leq a_{0}$. Let

$$
\mathcal{D}_{\lambda}=\left\{\xi \in U:\left|\nabla \varphi_{m}(\xi)\right|_{g} \leq \sqrt{2}|\log (\lambda-8 \pi m)|^{-3}\right\}
$$

and consider the interval

$$
I_{\lambda}=\left[\frac{m_{0}}{\sqrt{|\log (\lambda-8 \pi m)|}}, M\right]
$$

where

$$
0<m_{0}=\inf _{\xi \in U}|A(\xi)|^{-\frac{1}{2}}, \quad M=2 \sup _{\left\{\xi \in U:|A(\xi)| \leq a_{0}\right\}} B^{-\frac{1}{2}}(\xi)<+\infty .
$$

For $\lambda$ close to $8 \pi m$ and for all $\xi \in \mathcal{D}_{\lambda}$ we have that

$$
\left.\frac{\left(\delta \partial_{\delta} E_{\lambda}\right)(\sqrt{\lambda-8 \pi m} \mu, \xi)}{\lambda-8 \pi m}\right|_{\mu=\frac{m_{0}}{\sqrt{\log (\lambda-8 \pi m) \mid}}}=2-A(\xi) m_{0}^{2}(1+o(1))-2 B(\xi) \frac{m_{0}^{2}}{|\log (\lambda-8 \pi m)|}+o(1)>0
$$

in view of $A(\xi) m_{0}^{2} \leq 1$, and

$$
\left.\frac{\left(\delta \partial_{\delta} E_{\lambda}\right)(\sqrt{\lambda-8 \pi m} \mu, \xi)}{\lambda-8 \pi m}\right|_{\mu=M}=2-A(\xi) M^{2}|\log (\lambda-8 \pi m)|(1+o(1))-2 B(\xi) M^{2}+o(1)<0
$$

since either $A(\xi) \geq a_{0}$ or $0 \leq A(\xi) \leq a_{0}, B(\xi) M^{2} \geq 4$. Moreover, in $I_{\lambda} \times \mathcal{D}_{\lambda}$ we have that

$$
\frac{\left(\delta^{2} \partial_{\delta \delta} E_{\lambda}\right)(\sqrt{\lambda-8 \pi m} \mu, \xi)}{\lambda-8 \pi m}=-2-A(\xi) \mu^{2}|\log (\lambda-8 \pi m)|(1+o(1))-2 B(\xi) \mu^{2}+o(1) \leq-1
$$

since either $A(\xi) \geq a_{0}$ or $0 \leq A(\xi) \leq a_{0}, B(\xi)>0$. So, for all $\lambda$ close to $8 \pi m$ and $\xi \in \mathcal{D}_{\lambda}$ there exists an unique $\mu(\lambda, \xi) \in \operatorname{Int} I_{\lambda}$ so that $\delta(\lambda, \xi):=\sqrt{\lambda-8 \pi m} \mu(\lambda, \xi)$ satisfies $\partial_{\delta} E_{\lambda}(\delta(\lambda, \xi), \xi)=0$. Moreover, by the IFT the map $\xi \in \mathcal{D}_{\lambda} \rightarrow \delta(\lambda, \xi)$ is a $C^{1}$-function of $\xi$ with

$$
\partial_{\xi} \delta(\lambda, \xi)=-\frac{\partial_{\delta \xi} E_{\lambda}(\delta(\lambda, \xi), \xi)}{\partial_{\delta \delta} E_{\lambda}(\delta(\lambda, \xi), \xi)}=O\left(|\log (\lambda-8 \pi m)|^{-3}\right),
$$


in view of $\mu^{2}(\lambda, \xi)\left|\partial_{\delta \delta} E_{\lambda}(\sqrt{\lambda-8 \pi m} \mu(\lambda, \xi), \xi)\right| \geq 1$ and $\partial_{\delta \xi} E_{\lambda}(\delta, \xi)=O\left(\delta|\log \delta|+|\log \delta|^{3}\left|\nabla \varphi_{m}(\xi)\right|_{g}^{2}\right)$ (as it can be easily shown by the methods in the proof of Theorem 3.2).

The aim now is to extend the map $\delta(\lambda, \xi)$ to the whole $U$ in a $C^{1}$-way. Letting $\eta \in C_{0}^{\infty}[-2,2]$ be a cut-off function so that $\eta=1$ in $[-1,1]$, we define the $C^{1}$-extension $\tilde{\delta}$ of $\delta$ to $\mathcal{D}$ as

$\tilde{\delta}(\lambda, \xi)=\eta\left(|\log (\lambda-8 \pi m)|^{6}\left|\nabla \varphi_{m}(\xi)\right|_{g}^{2}\right) \delta(\lambda, \xi)+\sqrt{\lambda-8 \pi m}\left[1-\eta\left(|\log (\lambda-8 \pi m)|^{6}\left|\nabla \varphi_{m}(\xi)\right|_{g}^{2}\right)\right]$

and $\tilde{E}_{\lambda}(\xi)=E_{\lambda}(\tilde{\delta}(\lambda, \xi), \xi)$. Since $\left|\partial_{\xi} \tilde{\delta}(\lambda, \xi)\right|=O\left(|\log (\lambda-8 \pi m)|^{-3}\right)$, by Theorem 4.4 we have that

$$
\tilde{E}_{\lambda}(\xi)=-8 \pi m-\lambda \log (\pi m)-32 \pi^{2} \varphi_{m}(\xi)+O(|\lambda-8 \pi m||\log (\lambda-8 \pi m)|)
$$

and

$\nabla_{\xi} \tilde{E}_{\lambda}(\xi)=\nabla_{\xi} E_{\lambda}(\tilde{\delta}(\lambda, \xi), \xi)+\partial_{\delta} E_{\lambda}(\tilde{\delta}(\lambda, \xi), \xi) \partial_{\xi} \tilde{\delta}(\lambda, \xi)=-32 \pi^{2} \nabla \varphi_{m}(\xi)+O\left(\sqrt{\lambda-8 \pi m}|\log (\lambda-8 \pi m)|^{2}\right)$

uniformly in $\xi \in U$. Since $\mathcal{D}$ is a stable critical set of $\varphi_{m}$ (according to Definition 1.2), we find a critical point $\xi_{\lambda} \in U$ of $\tilde{E}_{\lambda}(\xi)+8 \pi m+\lambda \log (\pi m)$, which is also a c.p. of $\tilde{E}_{\lambda}(\xi)$. By $\nabla_{\xi} \tilde{E}_{\lambda}\left(\xi_{\lambda}\right)=0$ we get that

$$
\nabla \varphi_{m}\left(\xi_{\lambda}\right)=O\left(\sqrt{\lambda-8 \pi m}|\log (\lambda-8 \pi m)|^{2}\right)
$$

and then $\xi_{\lambda} \in \mathcal{D}_{\lambda}$. Moreover $\tilde{\delta}(\lambda, \xi)=\delta(\lambda, \xi)$ satisfies $\partial_{\delta} E_{\lambda}\left(\delta\left(\lambda, \xi_{\lambda}\right), \xi_{\lambda}\right)=0$, and then $\nabla_{\xi} \tilde{E}_{\lambda}\left(\xi_{\lambda}\right)=0$ is equivalent to $\nabla_{\xi} E_{\lambda}\left(\delta\left(\lambda, \xi_{\lambda}\right), \xi_{\lambda}\right)=0$. In conclusion, up to take $U$ smaller so that $\nabla \varphi_{m}(\xi) \neq 0$ for all $\xi \in U \backslash \mathcal{D}$, the pair $\left(\delta\left(\lambda, \xi_{\lambda}\right), \xi_{\lambda}\right)$ is a c.p. of $E_{\lambda}(\delta, \xi)$ and, along a sub-sequence, $\xi_{\lambda} \rightarrow q \in \mathcal{D}$ as $\lambda \rightarrow 8 \pi m$. By construction, the corresponding solution has the required asymptotic properties.

Remark 4.5. i) The validity of condition (13) just on $\mathcal{D}$ is enough to provide Theorem 1.5 in the case of $\mathcal{D}=\left\{\xi_{0}\right\}$, where $\xi_{0}$ is a non-degenerate local minimum/maximum point of $\varphi_{m}$. In this case, we just consider a small ball $B_{s_{\lambda}}\left(\xi_{0}\right)$ as $\mathcal{D}_{\lambda}$, with $s_{\lambda}=|\log (\lambda-8 \pi m)|^{-3}$. Since $A\left(\xi_{0}\right) \geq 0$ and $\nabla \varphi_{m}\left(\xi_{0}\right)=0$ we have that $A(\xi) \geq-C_{0} s_{\lambda}$ and $\left|\nabla \varphi_{m}(\xi)\right|_{g} \leq C_{0} s_{\lambda}$ for all $\xi \in B_{s_{\lambda}}\left(\xi_{0}\right)$ and some $C_{0}>0$. Since $B(\xi)>0$ for all $\xi \in B_{s_{\lambda}}\left(\xi_{0}\right)$ if $A\left(\xi_{0}\right)=0$, it is easy to see as before that for $\lambda$ close to $8 \pi m$ and for all $\xi \in B_{s_{\lambda}}\left(\xi_{0}\right)$

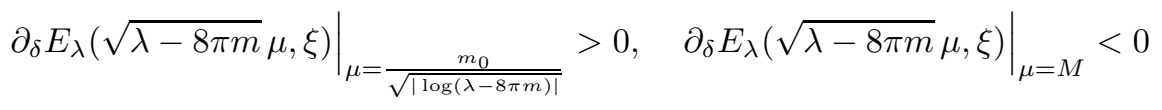

with

$$
\partial_{\delta \delta} E_{\lambda}(\sqrt{\lambda-8 \pi m} \mu, \xi) \leq-\frac{1}{\mu^{2}}
$$

in $I_{\lambda} \times B_{s_{\lambda}}\left(\xi_{0}\right)$. So, for all $\lambda$ close to $8 \pi m$ we can still find a $C^{1}-\operatorname{map} \xi \in B_{s_{\lambda}}\left(\xi_{0}\right) \rightarrow \delta(\lambda, \xi)$ so that $\partial_{\delta} E_{\lambda}(\delta(\lambda, \xi), \xi)=0$. Setting $\tilde{E}_{\lambda}(\xi)=E_{\lambda}(\delta(\lambda, \xi), \xi)$ for $\xi \in B_{s_{\lambda}}\left(\xi_{0}\right)$, by Theorem 4.4 we have that

$$
\tilde{E}_{\lambda}(\xi)=-8 \pi m-\lambda \log (\pi m)-32 \pi^{2} \varphi_{m}(\xi)+O(|\lambda-8 \pi m||\log (\lambda-8 \pi m)|) .
$$

Since by the non-degeneracy of $\xi_{0}$ we have on $\partial B_{s_{\lambda}}\left(\xi_{0}\right)$ that $\varphi_{m}(\xi) \geq \varphi_{m}\left(\xi_{0}\right)+C_{1} s_{\lambda}^{2} / \varphi_{m}(\xi) \leq$ $\varphi_{m}\left(\xi_{0}\right)-C_{1} s_{\lambda}^{2}$ for some $C_{1}>0$, we can find an interior minimum/maximum point $\xi_{\lambda} \in B_{s_{\lambda}}\left(\xi_{0}\right)$ of $\tilde{E}_{\lambda}(\xi)$ on $B_{s_{\lambda}}\left(\xi_{0}\right)$. By $\partial_{\delta} E_{\lambda}\left(\delta\left(\lambda, \xi_{\lambda}\right), \xi_{\lambda}\right)=0$, we also deduce that $\nabla_{\xi} E_{\lambda}\left(\delta\left(\lambda, \xi_{\lambda}\right), \xi_{\lambda}\right)=0$, and the pair $\left(\delta\left(\lambda, \xi_{\lambda}\right), \xi_{\lambda}\right)$ is the c.p. of $E_{\lambda}(\delta, \xi)$ we were searching for.

ii) If (13) does hold just in $\mathcal{D}$, Theorem 1.5 is also valid in the special case $|A(\xi)|=O\left(\left|\nabla \varphi_{m}(\xi)\right|_{g}\right)$. Indeed, condition (13) reduces to $B(\xi)>0(<0)$ on $\mathcal{D}$ and in $\mathcal{D}_{\lambda}$ we have that $A(\xi) \geq$ $-C_{0}|\log (\lambda-8 \pi m)|^{-3}$ for some $C_{0}>0$. Similarly as in point (i), it is still possible to define the map $\xi \in \mathcal{D}_{\lambda} \rightarrow \delta(\lambda, \xi)$, and the remaining argument in the proof of Theorem 1.5 works also in this case by extending $\delta(\lambda, \xi)$ on a small neighborhood $U$ of $\mathcal{D}$ in $\tilde{S}^{m} \backslash \Delta$. 


\section{Proof of Theorem 1.6}

In this section, we shall study the existence of non-topological solutions of (14). To this purpose we look for a solution to the equivalent problem (15) of the form $w=u+c_{-}(u)$ with $\int_{T} u=0$ and we are lead to study (16). Assume that $N$ is even, so that equation (16) is a perturbation of (11) $\lambda=8 \pi m$ with $m=\frac{N}{2}$. Notice that the energy functional of (15) is given by

$$
\tilde{I}_{\epsilon}(w)=\frac{1}{2} \int_{T}|\nabla w|^{2}+\frac{1}{2 \epsilon^{2}} \int_{T}\left(k e^{w}-1\right)^{2}+\frac{4 \pi N}{|T|} \int_{T} w, \quad w \in H^{1}(T) .
$$

Introduce the notation $C(u):=16 \pi N \frac{\int_{T} k^{2} e^{2 u}}{\left(\int_{T} k e^{u}\right)^{2}}$, so that $e^{c_{-}(u)}=\frac{8 \pi N \epsilon^{2}}{\int_{T} k e^{u}\left(1+\sqrt{1-\epsilon^{2} C(u)}\right)}$ and

$$
\begin{aligned}
I_{\epsilon}(u):=\tilde{I}_{\epsilon}\left(u+c_{-}(u)\right)= & J_{4 \pi N}(u)-4 \pi N \log \left(1+\sqrt{1-\epsilon^{2} C(u)}\right)-\frac{4 \pi N}{1+\sqrt{1-\epsilon^{2} C(u)}} \\
& +4 \pi N \log \left(8 \pi N \epsilon^{2}\right)+\frac{|T|}{2 \epsilon^{2}}-2 \pi N .
\end{aligned}
$$

Hence, if $u \in \mathcal{A}_{\epsilon}=\left\{u \in \bar{H} \mid \epsilon^{2} C(u) \leq 1\right\}$ is a critical point of $I_{\epsilon}$ with $\epsilon^{2} C(u)<1$, then $u+c_{-}(u)$ is a solution to (15) and $u$ is a solution to (16). Observe that $I_{\epsilon}$ is a perturbation of $J_{8 \pi m}$ as $\epsilon \rightarrow 0^{+}$, in view of $4 \pi N=8 \pi m$.

Given $m$ distinct points $\xi_{j} \in T \backslash\left\{p_{1}, \ldots, p_{l}\right\}, j=1, \ldots, m$, we will define $\delta_{j}$ according to (24) and assume

$$
\exists C>1: \epsilon \leq C \delta^{2} .
$$

Letting $W(x)=\sum_{j=1}^{m} W_{j}(x)$, we look for a solution of (16) in the form $u=W+\phi$, for some small remainder term $\phi$. In terms of $\phi$, problem (16) is equivalent to find $\phi \in \bar{H}$ so that $W+\phi \in \mathcal{A}_{\epsilon}$ and

$$
L^{\epsilon}(\phi)=-\left[R^{\epsilon}+N^{\epsilon}(\phi)\right] \quad \text { in } T .
$$

The linear operator $L^{\epsilon}$ is defined as

$$
L^{\epsilon}(\phi)=\Delta \phi+4 \pi N \frac{k e^{W}}{\int_{T} k e^{W}}\left(\phi-\frac{\int_{T} k e^{W} \phi}{\int_{T} k e^{W}}\right)+\Lambda^{\epsilon}(\phi),
$$

where

$$
\begin{aligned}
\Lambda^{\epsilon}(\phi)= & \frac{4 \pi N \epsilon^{2} C(W)}{\left(1+\sqrt{1-\epsilon^{2} C(W)}\right)^{2}}\left(\frac{k e^{W}}{\int_{T} k e^{W}}-\frac{2 k^{2} e^{2 W}}{\int_{T} k^{2} e^{2 W}}\right)\left[\phi-\frac{\int_{T} k e^{W} \phi}{\int_{T} k e^{W}}\right. \\
& \left.+\frac{\epsilon^{2} C(W)}{\left(1+\sqrt{1-\epsilon^{2} C(W)}\right) \sqrt{1-\epsilon^{2} C(W)}}\left(\frac{\int_{T} k^{2} e^{2 W} \phi}{\int_{T} k^{2} e^{2 W}}-\frac{\int_{T} k e^{W} \phi}{\int_{T} k e^{W}}\right)\right] \\
& +\frac{4 \pi N k e^{W}}{\int_{T} k e^{W}} \frac{\epsilon^{2} C(W)}{\left(1+\sqrt{1-\epsilon^{2} C(W)}\right) \sqrt{1-\epsilon^{2} C(W)}}\left(\frac{\int_{T} k^{2} e^{2 W} \phi}{\int_{T} k^{2} e^{2 W}}-\frac{\int_{T} k e^{W} \phi}{\int_{T} k e^{W}}\right) .
\end{aligned}
$$

Observe that $L^{\epsilon}$ is defined for all $\phi \in \bar{H}$. The nonlinear part $N^{\epsilon}$ is well-defined for $\phi \in \bar{H}$ such that $W+\phi \in \mathcal{A}_{\epsilon}$ and is given by

$$
\begin{aligned}
N^{\epsilon}(\phi)= & 4 \pi N\left(\frac{k e^{W+\phi}}{\int_{T} k e^{W+\phi}}-\frac{k e^{W} \phi}{\int_{T} k e^{W}}+\frac{k e^{W} \int_{T} k e^{W} \phi}{\left(\int_{T} k e^{W}\right)^{2}}-\frac{k e^{W}}{\int_{T} k e^{W}}\right)-\Lambda_{\epsilon}(\phi) \\
& +\frac{4 \pi N \epsilon^{2} C(W+\phi)}{\left(1+\sqrt{1-\epsilon^{2} C(W+\phi)}\right)^{2}}\left(\frac{k e^{W+\phi}}{\int_{T} k e^{W+\phi}}-\frac{k^{2} e^{2(W+\phi)}}{\int_{T} k^{2} e^{2(W+\phi)}}\right) \\
& -\frac{4 \pi N \epsilon^{2} C(W)}{\left(1+\sqrt{1-\epsilon^{2} C(W)}\right)^{2}}\left(\frac{k e^{W}}{\int_{T} k e^{W}}-\frac{k^{2} e^{2 W}}{\int_{T} k^{2} e^{2 W}}\right) .
\end{aligned}
$$


The approximation rate of $W$ becomes

$$
R^{\epsilon}=\Delta W+4 \pi N\left(\frac{k e^{W}}{\int_{T} k e^{W}}-\frac{1}{|T|}\right)+\frac{4 \pi N \epsilon^{2} C(W)}{\left(1+\sqrt{1-\epsilon^{2} C(W)}\right)^{2}}\left(\frac{k e^{W}}{\int_{T} k e^{W}}-\frac{k^{2} e^{2 W}}{\int_{T} k^{2} e^{2 W}}\right) .
$$

We have that

Lemma 5.1. Let $N$ be an even number and $m=\frac{N}{2}$. Assume (24) and (65). There exists a constant $C>0$, independent of $\delta>0$ small, such that for all $\xi \in \Xi$

$$
\left\|R^{\epsilon}\right\|_{*} \leq C\left(\delta\left|\nabla \varphi_{m}(\xi)\right|_{g}+\delta^{2-\sigma}\right) .
$$

Proof: First, note that $R^{\epsilon}=R_{8 \pi m}+\frac{8 \pi m \epsilon^{2} C(W)}{\left(1+\sqrt{1-\epsilon^{2} C(W)}\right)^{2}}\left(\frac{k e^{W}}{\int_{T} k e^{W}}-\frac{k^{2} e^{2 W}}{\int_{T} k^{2} e^{2 W}}\right)$ in view of $N=2 m$. As in (31) we have that

$$
\begin{aligned}
\int_{T} k^{2} e^{2 W} d x & =\sum_{j=1}^{m} \frac{1}{64 \delta_{j}^{4}} \int_{B_{r_{0}}\left(\xi_{j}\right)} \rho_{j}^{2}(x) e^{2 U_{j}}\left(1+O\left(\delta^{2}|\log \delta|\right)\right) d x+O(1) \\
& =\sum_{j=1}^{m} \frac{1}{64 \delta_{j}^{4}}\left(\frac{64 \pi \rho_{j}^{2}\left(\xi_{j}\right)}{3 \delta_{j}^{2}}+O(|\log \delta|)\right)+O(1)=\frac{\pi}{3 \delta^{6}} \sum_{j=1}^{m} \frac{1}{\rho_{j}\left(\xi_{j}\right)}\left(1+O\left(\delta^{2}|\log \delta|\right)\right) .
\end{aligned}
$$

Hence, in $T \backslash \cup_{j=1}^{m} B_{r_{0}}\left(\xi_{j}\right)$ there holds $\frac{k^{2} e^{2 W}}{\int_{T} k^{2} e^{2 W}}=O\left(\delta^{6}\right)$ in view of $W(x)=O(1)$, and in $B_{r_{0}}\left(\xi_{j}\right)$, $j \in\{1, \ldots, m\}$, there holds

$$
\frac{k^{2} e^{2 W}}{\int_{T} k^{2} e^{2 W}}=\frac{3 \delta^{2}\left[\rho_{j}^{2}(x)+O\left(\delta^{2}|\log \delta|\right)\right]}{64 \pi \rho_{j}^{2}\left(\xi_{j}\right) \sum_{l=1}^{m}\left[\rho_{l}\left(\xi_{l}\right)\right]^{-1}\left(1+O\left(\delta^{2}|\log \delta|\right)\right)} e^{2 U_{j}}=O\left(\delta^{2} e^{U_{j}}\right),
$$

which summarize as follows: $\frac{k^{2} e^{2 W}}{\int_{T} k^{2} e^{2 W}}=O\left(\delta^{2} \sum_{j=1}^{m} \chi_{j} e^{U_{j}}+\delta^{6} \chi_{T \backslash \cup_{j=1}^{m} B_{r_{0}}\left(\xi_{j}\right)}\right)$. On the other hand, from (31) we get that

$$
C(W)=16 \pi N \frac{\frac{\pi}{3 \delta^{6}} \sum_{j=1}^{m} \frac{1}{\rho_{j}\left(\xi_{j}\right)}\left(1+O\left(\delta^{2}|\log \delta|\right)\right)}{\left[\frac{\pi m}{\delta^{2}}+O(|\log \delta|)\right]^{2}}=\frac{32}{3 m \delta^{2}} \sum_{j=1}^{m} \frac{1}{\rho_{j}\left(\xi_{j}\right)}\left(1+O\left(\delta^{2}|\log \delta|\right)\right),
$$

which implies by (65) that for $\epsilon$ and $\delta$ sufficiently small $W \in \mathcal{A}_{\epsilon}$ and

$$
\frac{8 \pi m \epsilon^{2} C(W)}{\left(1+\sqrt{1-\epsilon^{2} C(W)}\right)^{2}}=2 \pi m \epsilon^{2} C(W)+O\left(\left[\epsilon^{2} C(W)\right]^{2}\right)=O\left(\frac{\epsilon^{2}}{\delta^{2}}\right) .
$$

Therefore, by using (32) and the estimate on $\frac{k^{2} e^{2 W}}{\int_{T} k^{2} e^{2 W}}$ we find the following estimate

$$
R^{\epsilon}-R_{8 \pi m}=O\left(\frac{\epsilon^{2}}{\delta^{2}}\left[\sum_{j=1}^{m} \chi_{j} e^{U_{j}}+\delta^{2}\right]\right)
$$

and then $\left\|R^{\epsilon}-R_{8 \pi m}\right\|_{*}=O\left(\epsilon^{2} \delta^{-2}\right)$. Thus, in view of (33) and (65) the conclusion follows.

Now, we are going to establish the expansion of $I_{\epsilon}(W)$.

Theorem 5.2. Assume (24) and (65). The following expansion does hold

$$
I_{\epsilon}(W)=-16 \pi m+8 \pi m \log \left(8 \epsilon^{2}\right)+\frac{|T|}{2 \epsilon^{2}}-32 \pi^{2} \varphi_{m}(\xi)+A(\xi) \delta^{2} \log \delta-B(\xi) \delta^{2}+\tilde{B}(\xi) \frac{\epsilon^{2}}{\delta^{2}}+o\left(\delta^{2}\right)
$$

in $C^{2}(\mathbb{R})$ and $C^{1}(\Xi)$ as $\delta \rightarrow 0^{+}$, where $\varphi_{m}(\xi), A(\xi)$ and $B(\xi)$ are given by (5), (7) and (12), respectively, and

$$
\tilde{B}(\xi)=\frac{32 \pi}{3} \sum_{j=1}^{m} \frac{1}{\rho_{j}\left(\xi_{j}\right)}
$$


Proof: By (70) we have that

$$
\left.\left[1+\sqrt{1-\epsilon^{2} C(W)}\right]^{-1}=\frac{1}{2}+\frac{\epsilon^{2}}{8} C(W)+O\left(\frac{\epsilon^{4}}{\delta^{4}}\right), \quad \log \left(1+\sqrt{1-\epsilon^{2} C(W)}\right)=\log 2-\frac{\epsilon^{2}}{4} C(W)+O\left(\frac{\epsilon^{4}}{\delta^{4}}\right)\right) .
$$

Hence, by using (64) we find that

$$
I_{\epsilon}(W)=J_{8 \pi m}(W)+\pi m \epsilon^{2} C(W)+8 \pi m \log \left(8 \pi m \epsilon^{2}\right)+\frac{|T|}{2 \epsilon^{2}}-8 \pi m+O\left(\epsilon^{4} \delta^{-4}\right) .
$$

Thus, the expansion (71) follows by (39), (65) and $C(W)=\left[\pi m \delta^{2}\right]^{-1} \tilde{B}(\xi)\left[1+O\left(\delta^{2}|\log \delta|\right)\right]$ in view of (70). Finally, the expansions for the derivatives follow similarly as in the proof of Theorem 3.2, in view of

$$
\begin{aligned}
\partial_{\beta}\left[I_{\epsilon}(W)\right] & =\partial_{\beta}\left[J_{8 \pi m}(W)\right]+\frac{4 \pi m \epsilon^{2} \partial_{\beta}[C(W)]}{\left(1+\sqrt{1-\epsilon^{2} C(W)}\right)^{2}} \\
& =\partial_{\beta}\left[J_{8 \pi m}(W)\right]+\pi m \epsilon^{2} \partial_{\beta}[C(W)]+O\left(\epsilon^{4} C(W)\left|\partial_{\beta}[C(W)]\right|\right)
\end{aligned}
$$

for either $\beta=\left(\xi_{j}\right)_{i}$ or $\beta=\delta$, and

$$
\partial_{\delta \delta}\left[I_{\epsilon}(W)\right]=\partial_{\delta \delta}\left[J_{8 \pi m}(W)\right]+\pi m \epsilon^{2} \partial_{\delta \delta}[C(W)]+O\left(\epsilon^{4} C(W)\left|\partial_{\delta \delta}[C(W)]\right|+\epsilon^{4}\left|\partial_{\delta}[C(W)]\right|^{2}\right),
$$

by using (43), (51), (53) and the expansions for the derivatives of $C(W)$ in the line of (70).

Since $L^{\epsilon}$ and $N^{\epsilon}$ are small perturbations of $L_{8 \pi m}$ and $N_{8 \pi m}$ in view of $\left\|\Lambda^{\epsilon}(\phi)\right\|_{*}=O\left(\frac{\epsilon^{2}}{\delta^{2}}\|\phi\|_{\infty}\right)$ and $N^{\epsilon}(\phi)=N_{8 \pi m}(\phi)+O\left(\frac{\epsilon^{2}}{\delta^{2}}\|\phi\|_{\infty}^{2}\right)$, as for Proposition 4.2, in view of (69) it follows

Proposition 5.3. There exists $\delta_{0}>0$ so that for all $0<\delta \leq \delta_{0}, \xi \in \Xi$ problem

$$
\begin{cases}L^{\epsilon}(\phi)=-\left[R^{\epsilon}+N^{\epsilon}(\phi)\right]+c_{0} \Delta P Z+\sum_{i=1}^{2} \sum_{j=1}^{m} c_{i j} \Delta P Z_{i j} & \text { in } T \\ \int_{T} \phi \Delta P Z=\int_{T} \phi \Delta P Z_{i j}=0 & \forall i=1,2, j=1, \ldots, m\end{cases}
$$

admits a unique solution $\phi(\delta, \xi) \in \bar{H} \cap W^{2,2}(T)$ and $c_{0}(\delta, \xi), c_{i j}(\delta, \xi) \in \mathbb{R}, i=1,2$ and $j=$ $1, \ldots, m$, where $\delta_{j}>0$ are as in (24) and $N^{\epsilon}, R^{\epsilon}$ are given by (67), (68). Moreover, the map $(\delta, \xi) \mapsto\left(\phi(\delta, \xi), c_{0}(\delta, \xi), c_{i j}(\delta, \xi)\right)$ is twice-differentiable in $\delta$ and one-differentiable in $\xi$ with

$$
\|\phi\|_{\infty}+\frac{\delta}{|\log \delta|}\left[\left\|\partial_{\delta} \phi\right\|_{\infty}+\sum_{i, j}\left\|\partial_{\left(\xi_{j}\right)_{i}} \phi\right\|_{\infty}+\frac{\delta\left\|\partial_{\delta \delta} \phi\right\|_{\infty}}{|\log \delta|}\right] \leq C|\log \delta|\left(\delta\left|\nabla \varphi_{m}(\xi)\right|_{g}+\delta^{2-\sigma}\right) .
$$

Remark 5.4. Notice that if $\|\phi\|_{\infty} \leq \nu \delta|\log \delta|$ then $W+\phi \in \mathcal{A}_{\epsilon}$ for $\delta$ and $\epsilon$ small enough.

The function $\phi(\delta, \xi)$ will be a solution to (66) , namely, $W+\phi(\delta, \xi)$ will be a true solution of (16) if $\delta$ and $\xi$ are such that $c_{0}(\delta, \xi)=c_{i j}(\delta, \xi)=0$ for all $i=1,2$, and $j=1, \ldots, m$. Similarly to Lemma 4.3, this problem is equivalent to finding critical points of the reduced energy $\mathcal{E}^{\epsilon}(\delta, \xi)=I_{\epsilon}(W+\phi(\delta, \xi))$.

Theorem 5.5. Assume (24) and (65). The following expansion does hold

$\mathcal{E}^{\epsilon}(\delta, \xi)=-16 \pi m+8 \pi m \log \left(8 \epsilon^{2}\right)+\frac{|T|}{2 \epsilon^{2}}-32 \pi^{2} \varphi_{m}(\xi)+A(\xi) \delta^{2} \log \delta-B(\xi) \delta^{2}+\tilde{B}(\xi) \frac{\epsilon^{2}}{\delta^{2}}+o\left(\delta^{2}\right)+r^{\epsilon}(\delta, \xi)$

in $C^{2}(\mathbb{R})$ and $C^{1}(\Xi)$ as $\delta \rightarrow 0^{+}$, where $\varphi_{m}(\xi), A(\xi), B(\xi)$ and $\tilde{B}(\xi)$ are given by (5), (17), (12) and (72), respectively. The term $r^{\epsilon}(\delta, \xi)$ satisfies for some $C>0$ independent of $(\delta, \xi)$

$$
\left|r^{\epsilon}(\delta, \xi)\right|+\frac{\delta}{|\log \delta|}\left|\nabla r^{\epsilon}(\delta, \xi)\right|+\frac{\delta^{2}}{|\log \delta|^{2}}\left|\partial_{\delta \delta} r^{\epsilon}(\delta, \xi)\right| \leq C \delta^{2}|\log \delta|\left|\nabla \varphi_{m}(\xi)\right|_{g}^{2}
$$


Proof (of Theorem 1.6): Similarly to Theorem 1.5 to find a critical point of $\mathcal{E}^{\epsilon}(\delta, \xi)$ the key step is to get the existence of a function $\delta=\delta(\epsilon, \xi)=\sqrt{\epsilon} \mu(\epsilon, \xi)$ such that $\partial_{\delta} \mathcal{E}^{\epsilon}(\delta(\epsilon, \xi), \xi)=0$ in a small neighborhood of the critical set $\mathcal{D}$. Even if $A(\xi) \geq 0$ for all $\xi \in\left(T \backslash\left\{p_{1}, \ldots, p_{l}\right\}\right)^{m} \backslash \Delta$, this is possible in view of $\tilde{B}(\xi)>0$ and "the correct sign" $B(\xi)<0$ in $\mathcal{D}$. The argument is based on the same one explained in Remark 4.5.(ii) and uses the crucial smallness property of $A(\xi)$ near $\mathcal{D}: A(\xi)=O\left(\left|\nabla \varphi_{m}(\xi)\right|_{g}^{2}\right)$.

\section{Appendix A}

We consider the operator

$$
L_{8 \pi m}(\phi)=\Delta_{g} \phi+\frac{8 \pi m k e^{W}}{\int_{S} k e^{W} d v_{g}}\left(\phi-\frac{\int_{S} k e^{W} \phi d v_{g}}{\int_{S} k e^{W} d v_{g}}\right)
$$

for which we first address a-priori estimates when all the $c_{i j}$ 's vanish:

Proposition 6.1. There exists $\delta_{0}>0$ and $C>0$ so that, for all $0<\delta \leq \delta_{0}, h \in C(S)$ with $\int_{S} h d v_{g}=0, \xi \in \Xi$ and $\phi \in H_{0}^{1}(S) \cap W^{2,2}(S)$ a solution of (55) with $L=L_{8 \pi m}$ and $c_{0}=c_{i j}=0$, $i=1,2$ and $j=1, \ldots, m$, one has

$$
\|\phi\|_{\infty} \leq C|\log \delta|\|h\|_{*} .
$$

Proof: By contradiction, assume the existence of sequences $\delta \rightarrow 0$, points $\xi \in \Xi$ with $\xi \rightarrow \xi^{*}$, functions $h$ with $|\log \delta|\|h\|_{*}=o(1)$ and solutions $\phi$ with $\|\phi\|_{\infty}=1$. Recall that $\delta_{j}^{2}=\delta^{2} \rho_{j}\left(\xi_{j}\right)$.

Setting $\mathcal{K}=\frac{8 \pi m k e^{W}}{\int_{S} k e^{W} d v_{g}}$ and $\psi=\phi-\frac{\int_{S} k e^{W} \phi d v_{g}}{\int_{S} k e^{W} d v_{g}}$, we have that $\Delta_{g} \psi+\mathcal{K} \psi=h$ in $S$ and $\psi$ does satisfy the same orthogonality conditions as $\phi$.

Since $\left\|\psi_{n}\right\|_{\infty} \leq 2\left\|\phi_{n}\right\|_{\infty} \leq 2$ and $\Delta_{g} \psi=o(1)$ in $C_{\text {loc }}\left(S \backslash\left\{\xi_{1}^{*}, \ldots, \xi_{m}^{*}\right\}\right)$, we can assume that $\psi \rightarrow \psi_{\infty}$ in $C_{\mathrm{loc}}^{1}\left(S \backslash\left\{\xi_{1}^{*}, \ldots, \xi_{m}^{*}\right\}\right)$. Since $\psi_{\infty}$ is bounded, it extends to an harmonic function in $S$, and then $\psi_{\infty}=c_{0}:=-\lim \frac{\int_{S} k e^{W} \phi d v_{g}}{\int_{S} k e^{W} d v_{g}}$ in view of $\frac{1}{|S|} \int_{S} \psi d v_{g}=-\frac{\int_{S} k e^{W} \phi d v_{g}}{\int_{S} k e^{W} d v_{g}}$.

The function $\Psi_{j}=\psi\left(y_{\xi_{j}}^{-1}\left(\delta_{j} y\right)\right)$ satisfies $\Delta \Psi_{j}+\tilde{\mathcal{K}}_{j} \Psi_{j}=\tilde{h}_{j}$ in $B_{\frac{2 r_{0}}{\delta_{j}}}(0)$, where $\tilde{\mathcal{K}}_{j}=\delta_{j}^{2} \mathcal{K}\left(y_{\xi_{j}}^{-1}\left(\delta_{j} y\right)\right)$ and $\tilde{h}_{j}=\delta_{j}^{2} h\left(y_{\xi_{j}}^{-1}\left(\delta_{j} y\right)\right)$. Since $\left|\tilde{h}_{j}\right| \leq C\|h\|_{*}$ and $\tilde{\mathcal{K}}_{j}=\frac{8}{\left(1+|y|^{2}\right)^{2}}\left(1+O\left(\delta^{2}|\log \delta|\right)\right)$ uniformly in $B_{\frac{2 r_{0}}{\delta}}(0)$ in view of Lemma 2.1 and (38), up to a sub-sequence, by elliptic estimates $\Psi_{j} \rightarrow \Psi_{j, \infty}$ in $C_{\mathrm{loc}}^{1}\left(\mathbb{R}^{2}\right)$, where $\Psi_{j, \infty}$ is a bounded solution of $\Delta \Psi_{j, \infty}+\frac{8}{\left(1+|y|^{2}\right)^{2}} \Psi_{j, \infty}=0$ of the form $\Psi_{j, \infty}=$ $\sum_{i=0}^{2} a_{i j} Y_{i}$ (see for example [1]). Since $-\Delta_{g} P Z_{i j}=\chi_{j} e^{-\varphi_{j}} e^{U_{j}} Z_{i j}-\frac{1}{|S|} \int_{S} \chi_{j} e^{-\varphi_{j}} e^{U_{j}} Z_{i j} d v_{g}$ in view of (54) and $\Delta_{g}=e^{-\varphi_{j}} \Delta$ in $B_{2 r_{0}}\left(\xi_{j}\right)$ through $y_{\xi_{j}}$, we have that

$$
0=-\int_{S} \psi \Delta_{g} P Z_{i j}=32 \int_{\mathbb{R}^{2}} \Psi_{j} \frac{y_{i}}{\left(1+|y|^{2}\right)^{3}} d y-\frac{32}{|S|} \int_{\mathbb{R}^{2}} \frac{y_{i}}{\left(1+|y|^{2}\right)^{3}} d y \int_{S} \psi_{n}+O\left(\delta^{3}\right) .
$$

Since then $\int_{\mathbb{R}^{2}} \Psi_{j} \frac{y_{i}}{\left(1+|y|^{2}\right)^{3}} d y=0$, we deduce that $a_{1 j}=a_{2 j}=0$. By the other orthogonality condition $\int_{S} \psi \Delta_{g} P Z=0$ similarly we deduce that

$$
0=-\sum_{j=1}^{m} \int_{S} \psi \Delta_{g} P Z_{0 j} d v_{g}=16 \int_{\mathbb{R}^{2}} \Psi_{j} \frac{1-|y|^{2}}{\left(1+|y|^{2}\right)^{3}} d y-\frac{16}{|S|} \int_{\mathbb{R}^{2}} \frac{1-|y|^{2}}{\left(1+|y|^{2}\right)^{3}} d y \int_{S} \psi_{n}+O\left(\delta^{2}\right),
$$


which implies $\sum_{j=1}^{m} a_{0 j}=0$ in view of $\int_{\mathbb{R}^{2}} \frac{1-|y|^{2}}{\left(1+|y|^{2}\right)^{3}} d y=0$. By dominated convergence we have that

$$
\begin{aligned}
& \int_{S} G\left(y, \xi_{j}\right) \mathcal{K} \psi d v_{g}=-\frac{1}{2 \pi} \log \delta \int_{B_{r_{0}}\left(\xi_{j}\right)} \mathcal{K} \psi d v_{g}+\int_{\mathbb{R}^{2}}\left[-\frac{1}{2 \pi} \log |y|+H\left(\xi_{j}, \xi_{j}\right)\right] \frac{8}{\left(1+|y|^{2}\right)^{2}} \Psi_{j, \infty} d y \\
& +\sum_{i \neq j} G\left(\xi_{i}, \xi_{j}\right) \int_{\mathbb{R}^{2}} \frac{8}{\left(1+|y|^{2}\right)^{2}} \Psi_{j, \infty} d y+o(1)=-\frac{1}{2 \pi} \log \delta \int_{B_{r_{0}}\left(\xi_{j}\right)} \mathcal{K} \psi d v_{g}+4 a_{0 j}+o(1)
\end{aligned}
$$

in view of $\int_{\mathbb{R}^{2}} \log |y| \frac{1-|y|^{2}}{\left(1+|y|^{2}\right)^{3}} d y=-\frac{\pi}{2}$. In view of $\int_{S} \mathcal{K} \psi=0$ and

$$
\left|\int_{S} G\left(y, \xi_{j}\right) h d v_{g}\right| \leq C|\log \delta| \int_{S}|h| d v_{g}+\frac{\|h\|_{*}}{\delta^{2}}\left|\int_{B_{\delta}\left(\xi_{j}\right)} G\left(y, \xi_{j}\right) d v_{g}\right| \leq C^{\prime}|\log \delta|\|h\|_{*}=o(1),
$$

by the Green's representation formula

$$
\sum_{j=1}^{m} \Psi_{j}(0)=\sum_{j=1}^{m} \psi\left(\xi_{j}\right)=\frac{m}{|S|} \int_{S} \psi d v_{g}+\sum_{j=1}^{m} \int_{S} G\left(y, \xi_{j}\right)[\mathcal{K} \psi-h] d v_{g}=m c_{0}+4 \sum_{j=1}^{m} a_{0 j}+o(1)
$$

which gives $\sum_{j=1}^{m} a_{0 j}=m c_{0}+4 \sum_{j=1}^{m} a_{0 j}$ as $n \rightarrow+\infty$. Since $\sum_{j=1}^{m} a_{0 j}=0$, we get that $c_{0}=0$.

Following [33, let $P Z_{j} \in H_{0}^{1}(S)$ be s.t. $\Delta_{g} P Z_{j}=\chi_{j} \Delta_{g} Z_{j}-\frac{1}{|S|} \int_{S} \chi_{j} \Delta_{g} Z_{j} d v_{g}$ in $S$, where

$$
Z_{j}(x)=\beta_{j}\left(\frac{y_{\xi_{j}}(x)}{\delta_{j}}\right), \quad \beta_{j}(y)=\frac{4}{3}\left[2 \log \delta_{j}+\log \left(1+|y|^{2}\right)\right] \frac{1-|y|^{2}}{1+|y|^{2}}+\frac{8}{3} \frac{1}{1+|y|^{2}}
$$

satisfies $e^{\varphi_{j}} \Delta_{g} Z_{j}+e^{U_{j}} Z_{j}=e^{U_{j}} Z_{0 j}$ in $B_{2 r_{0}}\left(\xi_{j}\right)$. Since it is easily seen that $P Z_{j}=\chi_{j} Z_{j}+$ $\frac{16 \pi}{3} H\left(\cdot, \xi_{j}\right)+O\left(\delta^{2}|\log \delta|^{2}\right)$ uniformly in $S$, we test the equation of $\psi$ against $P Z_{j}$ to get:

$$
\begin{aligned}
& \int_{S} \psi\left[\chi_{j} \Delta_{g} Z_{j}-\frac{1}{|S|} \int_{S} \chi_{j} \Delta_{g} Z_{j} d v_{g}\right] d v_{g}+\int_{S} \mathcal{K} \psi P Z_{j} d v_{g}=\int_{S} \chi_{j} \psi\left[e^{\varphi_{j}} \Delta_{g} Z_{j}+\mathcal{K} Z_{j}\right] d v_{g}+o(1) \\
& =\int_{S} \chi_{j} \psi e^{U_{j}} Z_{0 j} d v_{g}+o(1)=16 \int_{\mathbb{R}^{2}} \Psi_{j} \frac{1-|y|^{2}}{\left(1+|y|^{2}\right)^{3}} d y+o(1)=\int_{S} h P Z_{j}=o(1)
\end{aligned}
$$

in view of $\int_{S} \mathcal{K} \psi d v_{g}=0, \int_{S} \psi d v_{g}=o(1), \int_{S} \chi_{j} \Delta_{g} Z_{j} d v_{g}=O(1), \int_{S} \chi_{j} \psi\left[\mathcal{K}-e^{U_{j}}\right] Z_{j} d v_{g}=$ $O\left(\delta^{2}|\log \delta|^{2}\right)$ and $\int_{S} h P Z_{j}=O\left(|\log \delta|\|h\|_{*}\right)=o(1)$. Since $\int_{\mathbb{R}^{2}} \Psi_{j} \frac{1-|y|^{2}}{\left(1+|y|^{2}\right)^{3}} d y=0$ we have that $a_{0 j}=0$. So far, we have shown that $\psi \rightarrow 0$ in $C_{\text {loc }}\left(S \backslash\left\{\xi_{1}^{*}, \ldots, \xi_{m}^{*}\right\}\right)$ and uniformly in $\cup_{j=1}^{m} B_{R \delta_{j}}\left(\xi_{j}\right)$, for all $R>0$.

Setting $\hat{\psi}_{j}(y)=\psi\left(y_{\xi_{j}}^{-1}(y)\right), \hat{\mathcal{K}}_{j}(y)=\mathcal{K}\left(y_{\xi_{j}}^{-1}(y)\right)$ and $\hat{h}_{j}(y)=h\left(y_{\xi_{j}}^{-1}(y)\right)$ for $y \in B_{2 r_{0}}(0)$, we have that $e^{\hat{\varphi}_{j}} \Delta \hat{\psi}_{j}+\hat{\mathcal{K}}_{j} \hat{\psi}_{j}=\hat{h}_{j}$. By now it is rather standard to show that the operator $\hat{L}_{j}=e^{\varphi_{j}} \Delta+\hat{\mathcal{K}}_{j}$ satisfies the maximum principle in $B_{r}(0) \backslash B_{R \delta_{j}}(0)$ for $R$ large and $r>0$ small enough, see for example [25]. As a consequence, we get that $\psi \rightarrow 0$ in $L^{\infty}(S)$. Since $\frac{\int_{S} k e^{W} \phi d v_{g}}{\int_{S} k e^{W} d v_{g}} \rightarrow c$ along a sub-sequence, $\|\psi\|_{\infty} \rightarrow 0$ implies $\phi \rightarrow c$ in $L^{\infty}(S)$ with $c=0$ in view of $\int_{S} \phi=0$, in contradiction with $\|\phi\|_{\infty}=1$. This completes the proof.

We are now ready for

Proof (of Proposition 4.1): Since $\left\|\Delta_{g} P Z_{i j}\right\|_{*} \leq C$ for all $i=0,1,2, j=1, \ldots, m$, and

$$
\left\|(\lambda-8 \pi m) \frac{k e^{W}}{\int_{S} k e^{W} d v_{g}}\left(\phi-\frac{\int_{S} k e^{W} \phi d v_{g}}{\int_{S} k e^{W} d v_{g}}\right)\right\|_{*}=O\left(|\lambda-8 \pi m|\|\phi\|_{\infty}\right),
$$


by Proposition 6.1 for $\lambda$ close to $8 \pi m$ any solution of (55) satisfies

$$
\|\phi\|_{\infty} \leq C|\log \delta|\left[\|h\|_{*}+\left|c_{0}\right|+\sum_{i=1}^{2} \sum_{j=1}^{m}\left|c_{i j}\right|\right] .
$$

To estimate the values of the $c_{i j}$ 's, test equation (55) against $P Z_{i j}, i=1,2$ and $j=1, \ldots, m$ :

$$
\int_{S} \phi L\left(P Z_{i j}\right) d v_{g}=\int_{S} h P Z_{i j} d v_{g}+c_{0} \sum_{l=0}^{m} \int_{S} \Delta_{g} P Z_{0 l} P Z_{i j} d v_{g}+\sum_{k=1}^{2} \sum_{l=1}^{m} c_{k l} \int_{S} \Delta_{g} P Z_{k l} P Z_{i j} d v_{g} .
$$

Since for $j=1, \ldots, m$ we have the following estimates in $C(S)$

$$
P Z_{i j}=\chi_{j} Z_{i j}+O(\delta), \quad i=1,2, \quad P Z_{0 j}=\chi_{j}\left(Z_{0 j}+2\right)+O\left(\delta^{2}|\log \delta|\right),
$$

it readily follows that $\int_{S} \Delta_{g} P Z_{k l} P Z_{i j} d v_{g}=-\frac{32 \pi}{3} \delta_{k i} \delta_{l j}+O(\delta)$, where the $\delta_{i j}$ 's are the Kronecker's symbols. By Lemma 2.1, (25), (38) and (74) we have that for $i=1,2$

$$
L\left(P Z_{i j}\right)=\chi_{j} \Delta_{g} Z_{i j}+e^{U_{j}} P Z_{i j}+O\left(\delta^{2}+\delta \sum_{k=1}^{m} e^{U_{k}}\right)=e^{U_{j}}\left[P Z_{i j}-e^{-\varphi_{j}} \chi_{j} Z_{i j}\right]+O\left(\delta^{2}+\delta \sum_{k=1}^{m} e^{U_{k}}\right)
$$

in view of $\frac{\int_{S} k e^{W} P Z_{i j} d v_{g}}{\int_{S} k e^{W} d v_{g}}=O(\delta)$, leading to $\left\|L\left(P Z_{i j}\right)\right\|_{*}=O(\delta)$. Similarly, we have that

$$
\begin{aligned}
L(P Z) & =\sum_{j=0}^{m}\left[\chi_{j} \Delta_{g} Z_{0 j}+e^{U_{j}} P Z_{0 j}-\frac{2}{m} \sum_{k=1}^{m} \chi_{k} e^{U_{k}}\right]+O\left(\delta^{2}\right)+O\left(\delta \sum_{k=1}^{m} e^{U_{k}}\right) \\
& =\sum_{j=0}^{m} e^{U_{j}}\left[P Z_{0 j}-\chi_{j} e^{-\varphi_{j}} Z_{0 j}-2 \chi_{j}\right]+O\left(\delta^{2}\right)+O\left(\delta \sum_{k=1}^{m} e^{U_{k}}\right)
\end{aligned}
$$

in view of $\frac{\int_{S} k e^{W} P Z_{0 j} d v_{g}}{\int_{S} k e^{W} d v_{g}}=\frac{2}{m}+O\left(\delta^{2}|\log \delta|\right)$, leading to $\|L(P Z)\|_{*}=O(\delta)$. Hence, we get that $\left|c_{0}\right|+\sum_{i=1}^{2} \sum_{j=1}^{m}\left|c_{i j}\right| \leq C\|h\|_{*}+\delta O\left(\|\phi\|_{\infty}+\left|c_{0}\right|+\sum_{i=1}^{2} \sum_{j=1}^{m}\left|c_{i j}\right|\right) \leq C^{\prime}\|h\|_{*}+\delta|\log \delta| O\left(\left|c_{0}\right|+\sum_{i=1}^{2} \sum_{j=1}^{m}\left|c_{i j}\right|\right)$,

yielding to the desired estimates $\|\phi\|_{\infty}=O\left(|\log \delta|\|h\|_{*}\right)$ and $\left|c_{0}\right|+\sum_{i=1}^{2} \sum_{j=1}^{m}\left|c_{i j}\right|=O\left(\|h\|_{*}\right)$. To prove the solvability assertion, problem (55) is equivalent to finding $\phi \in H$ such that

$$
\int_{S}\langle\nabla \phi, \nabla \psi\rangle_{g} d v_{g}=\int_{S}\left[\frac{\lambda k e^{W}}{\int_{S} k e^{W} d v_{g}}\left(\phi-\frac{\int_{S} k e^{W} \phi d v_{g}}{\int_{S} k e^{W} d v_{g}}\right)-h\right] \psi d v_{g} \quad \forall \psi \in H,
$$

where $H=\left\{\phi \in H_{0}^{1}(S): \int_{S} \phi \Delta_{g} P Z_{i j} d v_{g}=\int_{S} \phi \Delta_{g} P Z d v_{g}=0, i=1,2, j=1, \ldots, m\right\}$. With the aid of Riesz representation theorem, the Fredholm's alternative guarantees unique solvability for any $h$ provided that the homogeneous equation has only the trivial solution: for (55) with $h=0$, the a-priori estimate (56) gives that $\phi=0$.

So far, we have seen that, if $T(h)$ denotes the unique solution $\phi$ of (55), the operator $T$ is a continuous linear map from $\left\{h \in L^{\infty}(S): \int_{S} h d v_{g}=0\right\}$, endowed with the $\|\cdot\|_{*}$-norm, into $\left\{\phi \in L^{\infty}(S): \int_{S} \phi d v_{g}=0\right\}$, endowed with $\|\cdot\|_{\infty}$-norm. The argument below is heuristic but can be made completely rigourous. The operator $T$ and the coefficients $c_{0}, c_{i j}$ are differentiable 
w.r.t. $\xi_{l}, l=1, \ldots, m$, or $\delta$. Differentiating equation (55), we formally get that $X=\partial_{\beta} \phi$, where $\beta=\xi_{l}$ with $l=1, \ldots, m$ or $\beta=\delta$, satisfies $L(X)=\tilde{h}(\phi)+d_{0} \Delta_{g} P Z+\sum_{i, j} d_{i j} \Delta_{g} P Z_{i j}$, where

$$
\begin{aligned}
\tilde{h}(\phi)= & -\partial_{\beta}\left(\frac{\lambda k e^{W}}{\int_{S} k e^{W} d v_{g}}\right) \phi+\partial_{\beta}\left[\frac{\lambda k e^{W}}{\left(\int_{S} k e^{W} d v_{g}\right)^{2}}\right] \int_{S} k e^{W} \phi d v_{g}+\frac{\lambda k e^{W}}{\left(\int_{S} k e^{W} d v_{g}\right)^{2}} \int_{S} k e^{W} \partial_{\beta} W \phi d v_{g} \\
& +c_{0} \partial_{\beta}\left(\Delta_{g} P Z\right)+\sum_{i, j} c_{i j} \partial_{\beta}\left(\Delta_{g} P Z_{i j}\right)
\end{aligned}
$$

and $d_{0}=\partial_{\beta} c_{0}, d_{i j}=\partial_{\beta} c_{i j}$, and the orthogonality conditions become

$$
\int_{S} X \Delta_{g} P Z_{i j} d v_{g}=-\int_{S} \phi \partial_{\beta}\left(\Delta_{g} P Z_{i j}\right) d v_{g}, \quad \int_{S} X \Delta_{g} P Z d v_{g}=-\int_{S} \phi \partial_{\beta}\left(\Delta_{g} P Z\right) d v_{g} .
$$

Find now coefficients $b_{0}, b_{i j}$ so that $Y=X+b_{0} P Z+\sum_{k, l} b_{k l} P Z_{k l}$ satisfies the orthogonality conditions $\int_{S} Y \Delta_{g} P Z d v_{g}=\int_{S} Y \Delta_{g} P Z_{i j} d v_{g}=0$. The coefficients $b_{0}, b_{i j}$ have to satisfy an almost diagonal system, and are then well-defined with $\left|b_{0}\right|+\sum_{i j}\left|b_{i j}\right| \leq C \frac{|\log \delta|}{\delta}\|h\|_{*}$ in view of $\left\|\partial_{\beta}\left(\Delta_{g} P Z_{i j}\right)\right\|_{*} \leq \frac{C}{\delta}$. Hence, the function $X$ can be uniquely expressed as $X=T(f)-b_{0} P Z-$ $\sum_{i, j} b_{i j} P Z_{i j}$, where $f=\tilde{h}(\phi)+b_{0} L(P Z)+\sum_{i, j} b_{i j} L\left(P Z_{i j}\right)$. Moreover, since $\left\|\partial_{\beta} W\right\|_{\infty}+\left\|\partial_{\beta} \mathcal{K}\right\|_{*} \leq$ $\frac{C}{\delta},\|\mathcal{K}\|_{*} \leq C$ and $\left\|\partial_{\beta}\left[\frac{\mathcal{K}}{\int_{S} k e^{W} d v_{g}}\right]\right\|_{*} \leq \frac{C}{\delta}\left(\int_{S} k e^{W} d v_{g}\right)^{-1}$ we find that

$$
\|f\|_{*} \leq\|\tilde{h}(\phi)\|_{*}+\left|b_{0}\right|\|L(P Z)\|_{*}+\sum_{i, j}\left|b_{i j}\right|\left\|L\left(P Z_{i j}\right)\right\|_{*} \leq C \frac{|\log \delta|}{\delta}\|h\|_{*},
$$

and by (56) we deduce that for any first derivative

$$
\left\|\partial_{\beta} \phi\right\|_{\infty} \leq C\left[|\log \delta|\|f\|_{*}+\frac{\|\phi\|_{\infty}}{\delta}\right] \leq C^{\prime} \frac{|\log \delta|^{2}}{\delta}\|h\|_{*} .
$$

Differentiating once more in $\delta$ the equation satisfied by $\partial_{\delta} \phi$ and arguing as above, we finally obtain that $\left\|\partial_{\delta \delta} \phi\right\|_{\infty} \leq C \frac{\left\lfloor\left.\log \delta\right|^{3}\right.}{\delta^{2}}\|h\|_{*}$, and the proof is complete.

\section{Appendix B}

By Proposition 4.1 we now deduce the following.

Proof (of Proposition 4.2): In terms of the operator $T$, problem (58) takes the form $\mathcal{A}(\phi)=$ $\phi$, where $\mathcal{A}(\phi):=-T(R+N(\phi))$. Given $\nu>0$, let us consider the space

$$
\mathcal{F}_{\nu}=\left\{\phi \in C(S):\|\phi\|_{\infty} \leq \nu\left[\delta|\log \delta| \sum_{j=1}^{m}\left|\nabla \log \left(\rho_{j} \circ y_{\xi_{j}}^{-1}\right)(0)\right|+\delta^{2-\sigma}|\log \delta|^{2}\right]\right\} .
$$

Notice that if $\phi \in \mathcal{F}_{\nu}$ then $W+\phi \in \mathcal{A}_{\epsilon}$ for $\delta$ and $\epsilon$ small enough. Since in view of Lemma 2.1 and (38) we have

$$
\begin{aligned}
& \| \frac{\lambda k e^{W+\phi} \psi_{1} \psi_{2}}{\int_{S} k e^{W+\phi} d v_{g}}-\frac{\lambda k e^{W+\phi} \psi_{1} \int_{S} k e^{W+\phi} \psi_{2} d v_{g}}{\left(\int_{S} k e^{W+\phi} d v_{g}\right)^{2}}-\frac{\lambda k e^{W+\phi} \psi_{2} \int_{S} k e^{W+\phi} \psi_{1} d v_{g}}{\left(\int_{S} k e^{W+\phi} d v_{g}\right)^{2}} \\
& -\frac{\lambda k e^{W+\phi} \int_{S} k e^{W+\phi} \psi_{1} \psi_{2} d v_{g}}{\left(\int_{S} k e^{W+\phi} d v_{g}\right)^{2}}+2 \frac{\lambda k e^{W+\phi}\left(\int_{S} k e^{W+\phi} \psi_{1} d v_{g}\right)\left(\int_{S} k e^{W+\phi} \psi_{2} d v_{g}\right)}{\left(\int_{S} k e^{W+\phi} d v_{g}\right)^{3}}\left\|_{*} \leq C\right\| \psi_{1}\left\|_{\infty}\right\| \psi_{2} \|_{\infty},
\end{aligned}
$$


for any $\phi_{1}, \phi_{2} \in \mathcal{F}_{\nu}$ we obtain that $\left.\left\|N\left(\phi_{1}\right)-N\left(\phi_{2}\right)\right\|_{*} \leq C\left(\left\|\phi_{1}\right\|_{\infty}+\| \phi_{2}\right) \|_{\infty}\right)\left\|\phi_{1}-\phi_{2}\right\|_{\infty}$ and then

$$
\left.\left\|\mathcal{A}\left(\phi_{1}\right)-\mathcal{A}\left(\phi_{2}\right)\right\|_{\infty} \leq C|\log \delta|\left(\left\|\phi_{1}\right\|_{\infty}+\| \phi_{2}\right) \|_{\infty}\right)\left\|\phi_{1}-\phi_{2}\right\|_{\infty} \leq \frac{1}{2}\left\|\phi_{1}-\phi_{2}\right\|_{\infty}
$$

for $\delta$ small in view of Proposition 4.1 Moreover, we have that for any $\phi \in \mathcal{F}_{\nu}$

$\|\mathcal{A}(\phi)\|_{\infty} \leq C|\log \delta|\left(\|\phi\|_{\infty}^{2}+\|R\|_{*}\right) \leq C|\log \delta|\|\phi\|_{\infty}^{2}+C_{0}\left[\delta|\log \delta| \sum_{j=1}^{m}\left|\nabla \log \left(\rho_{j} \circ y_{\xi_{j}}^{-1}\right)(0)\right|+\delta^{2-\sigma}|\log \delta|^{2}\right]$

in view of Lemma 2.2. Then, for $\nu=2 C_{0}$ and $\delta$ small $\mathcal{A}$ is a contraction mapping of $\mathcal{F}_{\nu}$ into itself, and therefore has a unique fixed point $\phi \in \mathcal{F}_{\nu}$.

By the Implicit Function Theorem it follows that the map $(\delta, \xi) \rightarrow\left(\phi(\delta, \xi), c_{0}(\delta, \xi), c_{i j}(\delta, \xi)\right)$ is (at least) twice-differentiable in $\delta$ and one differentiable in $\xi$. Differentiating $\phi=-T(R+N(\phi))$ w.r.t. $\beta=\xi_{l}, l=1, \ldots, m$, or $\beta=\delta$, we get that $\partial_{\beta} \phi=-\partial_{\beta} T(R+N(\phi))-T\left(\partial_{\beta} R+\partial_{\beta} N(\phi)\right)$. By Lemma 2.2 and (57) we have that

$$
\left\|\partial_{\beta} T(R+N(\phi))\right\|_{\infty} \leq C \frac{|\log \delta|^{2}}{\delta}\left(\|R\|_{*}+\|N(\phi)\|_{*}\right)=O\left(|\log \delta|^{2} \sum_{j=1}^{m}\left|\nabla \log \left(\rho_{j} \circ y_{\xi_{j}}^{-1}\right)(0)\right|+\delta^{1-\sigma}|\log \delta|^{3}\right),
$$

and, in view of $\left\|\partial_{\beta} W\right\|_{\infty} \leq \frac{C}{\delta}$, we can estimate

$$
\begin{aligned}
& \partial_{\beta} N(\phi)=N(\phi) \partial_{\beta} W+\lambda\left(\frac{k e^{W+\phi}}{\int_{S} k e^{W+\phi} d v_{g}}-\frac{k e^{W}}{\int_{S} k e^{W} d v_{g}}\right) \partial_{\beta} \phi \\
& -\lambda\left(\frac{k e^{W+\phi} \int_{S} k e^{W+\phi} \partial_{\beta} W d v_{g}}{\left(\int_{S} k e^{W+\phi} d v_{g}\right)^{2}}-\frac{k e^{W} \int_{S} k e^{W} \partial_{\beta} W d v_{g}}{\left(\int_{S} k e^{W} d v_{g}\right)^{2}}-\frac{k e^{W} \phi \int_{S} k e^{W} \partial_{\beta} W d v_{g}}{\left(\int_{S} k e^{W} d v_{g}\right)^{2}}-\frac{k e^{W} \int_{S} k e^{W} \partial_{\beta} W \phi d v_{g}}{\left(\int_{S} k e^{W} d v_{g}\right)^{2}}\right. \\
& \left.+2 \frac{k e^{W}\left(\int_{S} k e^{W} \partial_{\beta} W d v_{g}\right)\left(\int_{S} k e^{W} \phi d v_{g}\right)}{\left(\int_{S} k e^{W} d v_{g}\right)^{3}}\right)-\lambda\left(\frac{k e^{W+\phi} \int_{S} k e^{W+\phi} \partial_{\beta} \phi d v_{g}}{\left(\int_{S} k e^{W+\phi} d v_{g}\right)^{2}}-\frac{k e^{W} \int_{S} k e^{W} \partial_{\beta} \phi d v_{g}}{\left(\int_{S} k e^{W} d v_{g}\right)^{2}}\right)
\end{aligned}
$$

as follows

$$
\begin{aligned}
\left\|\partial_{\beta} N(\phi)\right\|_{*} & \leq C\left[\left\|\partial_{\beta} W\right\|_{\infty}\|\phi\|_{\infty}^{2}+\|\phi\|_{\infty}\left\|\partial_{\beta} \phi\right\|_{\infty}\right] \\
& =O\left(\delta|\log \delta|^{2} \sum_{j=1}^{m}\left|\nabla \log \left(\rho_{j} \circ y_{\xi_{j}}^{-1}\right)(0)\right|^{2}+\delta^{3-2 \sigma}|\log \delta|^{4}\right)+o\left(\frac{\left\|\partial_{\beta} \phi\right\|_{\infty}}{|\log \delta|}\right) .
\end{aligned}
$$

Since $\int_{S} \chi_{j} e^{-\varphi_{j}} e^{U_{j}} d v_{g}=\int_{\mathbb{R}^{2}} \chi(|y|) \frac{8 \delta^{2} \rho_{j}\left(\xi_{j}\right)}{\left(\delta^{2} \rho_{j}\left(\xi_{j}\right)+|y|^{2}\right)^{2}} d y$, we have that

$$
\partial_{\xi_{l}}\left(\int_{S} \chi_{j} e^{-\varphi_{j}} e^{U_{j}} d v_{g}\right)=8 \partial_{\xi_{l}} \log \rho_{j}\left(\xi_{j}\right) \int_{\mathbb{R}^{2}} \frac{1-|y|^{2}}{\left(1+|y|^{2}\right)^{3}}+O\left(\delta^{2}\right)=O\left(\delta^{2}\right)
$$

and

$\partial_{\delta}\left(\int_{S} \chi_{j} e^{-\varphi_{j}} e^{U_{j}} d v_{g}\right)=\int_{\mathbb{R}^{2}} \chi(|y|) \frac{16 \delta \rho_{j}\left(\xi_{j}\right)\left(|y|^{2}-\delta^{2} \rho_{j}\left(\xi_{j}\right)\right)}{\left(\delta^{2} \rho_{j}\left(\xi_{j}\right)+|y|^{2}\right)^{3}} d y=\frac{16}{\delta} \int_{\mathbb{R}^{2}} \frac{|y|^{2}-1}{\left(1+|y|^{2}\right)^{3}} d y+O(\delta)=O(\delta)$.

Since $\varphi_{j}\left(\xi_{j}\right)=0$ and $\nabla \varphi_{j}\left(\xi_{j}\right)=0$, we have that $e^{-\varphi_{j}}=1+O\left(\left|y_{\xi_{j}}(x)\right|^{2}\right)$ and $\partial_{\beta}\left(\chi_{j} e^{-\varphi_{j}}(x)\right)=$ $O\left(\left|y_{\xi_{j}}(x)\right|\right)$, and then

$$
\Delta_{g} \partial_{\beta} W=-\sum_{j=1}^{m} \chi_{j} e^{U_{j}} \partial_{\beta} U_{j}+O\left(\delta^{1-\sigma}\right)
$$


in view of $\left|\partial_{\beta} U_{j}\right|=O\left(\frac{1}{\delta}\right)$, where the big $O$ is estimated in $\|\cdot\|_{*}$-norm. Since in $B_{r_{0}}\left(\xi_{j}\right)$

$\partial_{\xi_{l}} W=\partial_{\xi_{l}} U_{j}+O\left(\delta^{2}|\log \delta|+\left|y_{\xi_{j}}(x)\right|+\left|\nabla \log \left(\rho_{j} \circ y_{\xi_{j}}^{-1}\right)(0)\right|\right), \quad \partial_{\delta} W=\partial_{\delta} U_{j}-\frac{2}{\delta}+O(\delta|\log \delta|)$,

in the same line as Lemma 2.1 and

$$
\frac{\lambda k e^{W}}{\int_{S} k e^{W} d v_{g}}=\sum_{j=1}^{m} \chi_{j} e^{U_{j}}\left[1+O\left(\left|\nabla \log \left(\rho_{j} \circ y_{\xi_{j}}^{-1}\right)(0)\right|\left|y_{\xi_{j}}(x)\right|+\delta^{2}|\log \delta|\right)\right]+O\left(\delta^{2}\right)
$$

in view of (32), by (42) and (50) we deduce for

$$
\partial_{\beta} R=\Delta_{g} \partial_{\beta} W+\frac{\lambda k e^{W}}{\int_{S} k e^{W} d v_{g}}\left(\partial_{\beta} W-\frac{\int_{S} k e^{W} \partial_{\beta} W}{\int_{S} k e^{W} d v_{g}}\right)
$$

the estimate

$$
\left\|\partial_{\beta} R\right\|_{*}=O\left(\sum_{j=1}^{m}\left|\nabla \log \left(\rho_{j} \circ y_{\xi_{j}}^{-1}\right)(0)\right|+\delta^{1-\sigma}|\log \delta|\right) .
$$

Combining all the estimates, we then get that

$$
\left\|\partial_{\beta} \phi\right\|_{\infty}=O\left(|\log \delta|^{2} \sum_{j=1}^{m}\left|\nabla \log \left(\rho_{j} \circ y_{\xi_{j}}^{-1}\right)(0)\right|+\delta^{1-\sigma}|\log \delta|^{3}\right)+o\left(\left\|\partial_{\beta} \phi\right\|_{\infty}\right),
$$

which in turn provides the validity of (60). We proceed in the same way to obtain the estimate (61) on $\partial_{\delta \delta} \phi$, and the proof is complete.

Lemma 4.3 is rather standard and we will omit its proof. Since the problem has been reduced to find c.p.'s of the reduced energy $E_{\lambda}(\delta, \xi)=J_{\lambda}(W+\phi(\delta, \xi))$, where $J_{\lambda}$ is given by (4), the last key step is show that the main asymptotic term of $E_{\lambda}$ is given by $J_{\lambda}(W)$.

Proof (of Theorem 4.4): Write

$$
\begin{aligned}
J_{\lambda}(W+\phi)-J_{\lambda}(W) & =D J_{\lambda}(W)[\phi]+\frac{D^{2} J_{\lambda}(W)[\phi, \phi]}{2}+\int_{0}^{1} \int_{0}^{1}\left[D^{2} J_{\lambda}(W+t s \phi)-D^{2} J_{\lambda}(W)\right][\phi, \phi] t d s d t \\
& =-\frac{1}{2} \int_{S} R \phi d v_{g}+\frac{1}{2} \int_{S} N(\phi) \phi d v_{g}+\int_{0}^{1} \int_{0}^{1}\left[D^{2} J_{\lambda}(W+t s \phi)-D^{2} J_{\lambda}(W)\right][\phi, \phi] t d s d t
\end{aligned}
$$

since $D J_{\lambda}(W)(\phi)=-\int_{S} R \phi d v_{g}, D^{2} J_{\lambda}(W)[\phi, \phi]=-\int_{S} L(\phi) \phi d v_{g}$ and

$$
D J_{\lambda}(W)[\phi]+D^{2} J_{\lambda}(W)[\phi, \phi]=\int_{S} N(\phi) \phi d v_{g}
$$

in view of $\int_{S} \phi d v_{g}=0$ and (58). Since $\frac{1}{2} \int_{S} k e^{W} d v_{g} \leq \int_{S} k e^{W+t s \phi} d v_{g} \leq 2 \int_{S} k e^{W} d v_{g}$ and $\left|e^{W+t s \phi}-e^{W}\right| \leq C e^{W}\|\phi\|_{\infty}$, it is straighforward to see that

$$
\begin{aligned}
& \left|D J_{\lambda}(W)[\phi]+D^{2} J_{\lambda}(W)[\phi, \phi]\right|+\left|\int_{0}^{1} t d t \int_{0}^{1} d s\left[D^{2} J_{\lambda}(W+t s \phi)-D^{2} J_{\lambda}(W)\right][\phi, \phi]\right| \\
& =O\left(\|N(\phi)\|_{*}\|\phi\|_{\infty}+\|\phi\|_{\infty}^{3}\right)=O\left(\|\phi\|_{\infty}^{3}\right),
\end{aligned}
$$

and then we deduce that

$$
\left|J_{\lambda}(W+\phi)-J_{\lambda}(W)\right|=O\left(\|R\|_{*}\|\phi\|_{\infty}+\|\phi\|_{\infty}^{3}\right)=O\left(\delta^{2}|\log \delta|\left|\nabla \varphi_{m}(\xi)\right|^{2}+\delta^{3-\sigma}|\log \delta|^{2}\right)
$$


in view of (59) and $4 \pi \nabla_{\xi_{j}} \varphi_{m}(\xi)=\nabla \log \left(\rho_{j} \circ y_{\xi_{j}}^{-1}\right)(0)$. Differentiating w.r.t. $\beta=\xi_{l}, l=1, \ldots, m$, or $\beta=\delta$ we get that

$$
\begin{aligned}
\partial_{\beta}\left[J_{\lambda}(W+\phi)-J_{\lambda}(W)\right]= & -\frac{1}{2} \int_{S}\left[\partial_{\beta} R \phi+R \partial_{\beta} \phi\right] d v_{g}+\frac{1}{2} \int_{S}\left(\partial_{\beta}[N(\phi)] \phi+N(\phi) \partial_{\beta} \phi\right) d v_{g} \\
& +\int_{0}^{1} t d t \int_{0}^{1} d s \partial_{\beta}\left\{\left[D^{2} J_{\lambda}(W+t s \phi)-D^{2} J_{\lambda}(W)\right][\phi, \phi]\right\} .
\end{aligned}
$$

Since it is straightforward to see that

$$
\left|\int_{0}^{1} t d t \int_{0}^{1} d s \partial_{\beta}\left\{\left[D^{2} J_{\lambda}(W+t s \phi)-D^{2} J_{\lambda}(W)\right][\phi, \phi]\right\}\right|=O\left(\|\phi\|_{\infty}^{2}\left\|\partial_{\beta} \phi\right\|_{\infty}+\|\phi\|_{\infty}^{3}\left\|\partial_{\beta} W\right\|_{\infty}\right),
$$

by (75) we deduce that

$$
\begin{aligned}
\left|\partial_{\beta}\left[J_{\lambda}(W+\phi)-J_{\lambda}(W)\right]\right| & =O\left(\left\|\partial_{\beta} R\right\|_{*}\|\phi\|_{\infty}+\|R\|_{*}\left\|\partial_{\beta} \phi\right\|_{\infty}+\|\phi\|_{\infty}^{2}\left\|\partial_{\beta} \phi\right\|_{\infty}+\|\phi\|_{\infty}^{3}\left\|\partial_{\beta} W\right\|_{\infty}\right) \\
& =O\left(\left[\delta^{2}|\log \delta|\left|\nabla \varphi_{m}(\xi)\right|^{2}+\delta^{3-\sigma}|\log \delta|^{2}\right] \frac{|\log \delta|}{\delta}\right)
\end{aligned}
$$

in view of (59)-(60) and $\left\|\partial_{\beta} W\right\|_{\infty}=O\left(\frac{1}{\delta}\right)$. Arguing similarly for the second derivative in $\delta$, we get that

$$
\left|\partial_{\delta \delta}\left[J_{\lambda}(W+\phi)-J_{\lambda}(W)\right]\right|=O\left(\left[\delta^{2}|\log \delta|\left|\nabla \varphi_{m}(\xi)\right|^{2}+\delta^{3-\sigma}|\log \delta|^{2}\right] \frac{|\log \delta|^{2}}{\delta^{2}}\right) .
$$

Combining the previous estimates on the difference $J_{\lambda}(W+\phi)-J_{\lambda}(W)$ with the expansion of $J_{\lambda}(W)$ contained in Theorem [3.2, we deduce the validity of the expansion (62) with an error term which can be estimated (in $C^{2}(\mathbb{R})$ and $C^{1}(\Xi)$ ) like $o\left(\delta^{2}\right)+r_{\lambda}(\delta, \xi)$ as $\delta \rightarrow 0$, where $r_{\lambda}(\delta, \xi)$ does satisfy (63).

\section{Acknowledgements}

Part of this work was carried out while the second author was visiting the Department of Mathematics, University of "Roma Tre". He would like to express his deep gratitude to Prof. Esposito for the many stimulating discussions about these topics and the warm hospitality.

\section{References}

[1] S. Baraket, F. PACARD, Construction of singular limits for a semilinear elliptic equation in dimension 2. Calc. Var. Partial Differential Equations 6 (1998), 1-38.

[2] D. Bartolucci, C.C. Chen, C.-S. Lin, G. Tarantello, Profile of blow-up solutions to mean field equations with singular data. Comm. Partial Differential Equations 29 (2004), 1241-1265.

[3] D. Bartolucci, F. De Marchis, On the Ambjorn-Olesen electroweak condensates. J. Math. Phys. 53 (2012), 073704.

[4] D. Bartolucci, F. De Marchis, A. Malchiodi, Supercritical conformal metrics on surfaces with conical singularities. Int. Math. Res. Not. 24 (2011), 5625-5643.

[5] D. Bartolucci, C.-S. Lin, G. Tarantello, Uniqueness and symmetry results for solutions of a mean field equation on $\mathbb{S}^{2}$ via a new bubbling phenomenon. Comm. Pure Appl. Math. 64 (2011), no. 12, 1677-1730.

[6] D. Bartolucci, A. Malchiodi, An improved geometric inequality via vanishing moments, with applications to singular Liouville equations. Comm. Math. Phys. 322 (2013), 415-452.

[7] D. Bartolucci, G. Tarantello, Liouville type equations with singular data and their application to periodic multivortices for the electroweak theory. Comm. Math. Phys. 229 (2002), 3-47. 
[8] L.A. Caffarelli, Y. YAng, Vortex condensation in the Chern-Simons-Higgs model: an existence theorem. Comm. Math. Phys. 168 (1995), 321-336.

[9] E. Caglioti, P.-L. Lions, C. Marchioro, M. Pulvirenti, A special class of stationary flows for two-dimensional Euler equations: a statistical mechanics description. Comm. Math. Phys. 143 (1992), 501-525.

[10] E. Caglioti, P.-L. Lions, C. Marchioro, M. Pulvirenti, A special class of stationary flows for two-dimensional Euler equations: a statistical mechanics description. II. Comm. Math. Phys. 174 (1995), 229-260.

[11] S-Y.A. Chang, C.C. Chen, C.-S. Lin, Extremal functions for a mean field equation in two dimension. Lectures on partial differential equations, 61-93, New Stud. Adv. Math., 2, Int. Press, Somerville, MA, 2003.

[12] S-Y.A. Chang, M.J. Gursky, P.C. YAng, The scalar curvature equation on 2- and 3-spheres. Calc. Var. Partial Differential Equations 1 (1993), 205-229.

[13] S-Y.A. Chang, P.C. YAng, Prescribing Gaussian curvature on $S^{2}$. Acta Math. 159 (1987), 215259.

[14] S. Chanillo, M. Kiessling, Rotational symmetry of solutions of some nonlinear problems in statistical mechanics and in geometry. Comm. Math. Phys. 160 (1994), 217-238.

[15] C.C. Chen, C.-S. Lin, Sharp estimates for solutions of multi-bubbles in compact Riemann surfaces. Comm. Pure Appl. Math. 55 (2002), 728-771.

[16] C.C. Chen, C.-S. Lin, Topological degree for a mean field equation on Riemann surfaces. Comm. Pure Appl. Math. 56 (2003), 1667-1727.

[17] C.C. Chen, C.-S. Lin, Mean field equation of Liouville type with singular data: sharper estimates. Discr. Cont. Dyn. Syst. 28 (2010), 1237-1272.

[18] C.C. Chen, C.-S. Lin In preparation.

[19] C.C. Chen, C.-S. Lin, G. WAng, Concentration phenomena of two-vortex solutions in a ChernSimons model. Ann. Sc. Norm. Sup. Pisa Cl. Sci. (5) 3 (2004), 367-397.

[20] S.-S. Chern, An elementary proof of the existence of isothermal parameters on a surface. Proc. Amer. Math. Soc. 6 (1955), 771-782.

[21] T. D'Aprile, Multiple blow-up solutions for the Liouville equation with singular data . Comm. Partial Differential Equations 38 (2013), 1409-1436.

[22] T. D'Aprile, P. Esposito In preparation.

[23] M. del Pino, P. Esposito, P. Figueroa, M. Musso, Non-topological condensates for the self-dual Chern-Simons model. Preprint, arXiv: 1305.5863.

[24] M. Del Pino, P. Esposito, M. Musso, Two-dimensional Euler flows with concentrated vorticities. Trans. Am. Math. Soc. 362 (2010), 6387-6395.

[25] M. del Pino, M. Kowalczyk, M. Musso, Singular limits in Liouville-type equations. Calc. Var. Partial Differential Equations 24 (2005), 47-81.

[26] F. De Marchis, Multiplicity result for a scalar field equation on compact surfaces. Comm. Partial Differential Equations 33 (2008), 2208-2224.

[27] F. De Marchis, Generic multiplicity for a scalar field equation on compact surfaces. J. Funct. Anal. 259 (2010), 2165-2192.

[28] W. Ding, J. Jost, J. LI, G. WANG, The differential equation $\Delta u=8 \pi-8 \pi h e^{u}$ on a compact Riemann surface. Asian J. Math. 1 (1997), 230-248.

[29] W. Ding, J. Jost, J. Li, G. Wang, Existence results for mean field equations. Ann. Inst. H. Poincaré Anal. Non Linéaire 16 (1999), 653-666.

[30] Z. DJADLI, Existence result for the mean field problem on Riemann surfaces of all genuses. Commun. Contemp. Math. 10 (2008), 205-220.

[31] Z. Djadli, A. Malchiodi, Existence of conformal metrics with constant Q-curvature. Ann. of Math. (2) 168 (2008), 813-858. 
[32] G. Dunne, Selfdual Chern-Simons theories. Lecture Notes in Physics Monograph Series 36. Springer, Heidelberg, 1995.

[33] P. Esposito, M. Grossi, A. Pistoia, On the existence of blowing-up solutions for a mean field equation. Ann. Inst. H. Poincaré Anal. Non Linéaire 22 (2005), 227-257.

[34] P. FigueroA, Singular limits for Liouville-type equations on the flat torus. Calc. Var. Partial Differential Equations 49 (2014), no. 1-2, 613-647.

[35] J.L. Kazdan, F.W. WARner, Curvature functions for compact 2-manifolds. Ann. of Math. (2) 99 (1974), 14-47.

[36] M.K.-H. KiEssLing, Statistical mechanics of classical particles with logarithmic interactions. Comm. Pure Appl. Math. 46 (1993), 27-56.

[37] Y.Y. LI, On a singularly perturbed elliptic equation. Adv. Differential Equations 2 (1997), 955-980.

[38] Y.Y. LI, Harnack type inequality: the method of moving planes. Comm. Math. Phys. 200 (1999), 421-444.

[39] C.-S. Lin, Topological degree for mean field equations on $S^{2}$. Duke Math. J. 104 (2000), 501-536.

[40] C.-S. Lin, C.-L. WANG, Elliptic functions, Green functions and the mean field equation on tori. Ann. of Math. (2) 172 (2010), 911-954.

[41] C.-S. LIN, S. YAN, Bubbling solutions for relativistic abelian Chern-Simons model on a torus. Comm. Math. Phys. 297 (2010), 733-758.

[42] C.-S. Lin, S. YAn, Existence of bubbling solutions for Chern-Simons model on a torus. Arch. Ration. Mech. Anal. 207 (2013), no. 2, 353-392.

[43] A. Malchiodi, Morse theory and a scalar field equation on compact surfaces. Adv. Differential Equations 13 (2008), 1109-1129.

[44] A. Malchiodi, D. RuIz, New improved Moser-Trudinger inequalities and singular Liouville equations on compact surfaces. G.A.F.A. 21 (2011), 1196-1217.

[45] M. Nolasco, G. Tarantello, On a sharp Sobolev-type inequality on two dimensional compact manifolds. Arch. Ration. Mech. Anal. 154 (1998), 161-195.

[46] M. Nolasco, G. Tarantello, Double Vortex condensates in the Chern-Simons-Higgs theory. Cal. Var. Partial Differential Equations 9 (1999), 31-94.

[47] M. Struwe, G. Tarantello, On multivortex solutions in Chern-Simons gauge theory. Boll. Unione Mat. Ital. Sez. B Artic. Ric. Mat. (8) 1 (1998), 109-121.

[48] G. Tarantello, Multiple condensate for Chern-Simons-Higgs theory. J. Math. Phys. 37 (1996), 3769-3796.

[49] G. TARANTELlo, Self-dual gauge field vortices: an analytical approach. Berlin-Heidelberg-NewYork, Springer, 2007

[50] G. Tarantello, Analytical, geometrical and topological aspects of a class of mean field equations on surfaces. Discrete Contin. Dyn. Syst. 28 (2010), no. 3, 931-973. 